\title{
Aproximação da Norma de Corte via Desigualdade de Grothendieck
}

\author{
Eric Ossami Endo \\ DisSERTAÇÃO APRESENTADA \\ AO \\ Instituto De Matemática e Estatística \\ DA \\ Universidade DE SÃo Paulo \\ PARA \\ OBTENÇÃO DO TÍTULO \\ $\mathrm{DE}$ \\ Mestre em CiÊnCIAS \\ Programa: Matemática \\ Orientador: Prof. Dr. Yoshiharu Kohayakawa
}

Durante o desenvolvimento deste trabalho o autor recebeu auxílio financeiro da CNPq

São Paulo, setembro de 2014 


\section{Aproximação da Norma de Corte via Desigualdade de Grothendieck}

Esta versão da dissertação contém as correções e alterações sugeridas pela Comissão Julgadora durante a defesa da versão original do trabalho, realizada em 17/07/2014. Uma cópia da versão original está disponível no Instituto de Matemática e Estatística da Universidade de São Paulo.

Comissão Julgadora:

- Prof. Dr. Yoshiharu Kohayakawa - IME-USP

- Prof. Dr. Rodrigo Bissacot Proença - IME-USP

- Prof. Dr. Carlos Hoppen - IM-UFRGS 


\section{Agradecimentos}

Agradeço primeiro ao Prof. Dr. Yoshiharu Kohayakawa, pela orientação desde a iniciação científica, sempre me mostrando quão interessante e bela é a matemática e a combinatória.

Agradeço fortemente a todos os professores que tive desde a minha infância até hoje, pois eles que me deram a base para a formação profissional e educacional do meu futuro.

Deixo um grande agradecimento aos meus amigos, Guilherme de Oliveira Mota, Luis Fernando Ragognette e Max Reinhold Jahnke, por terem tempo e paciência para me ajudar nesta dissertação e terem me concedido dicas valiosas.

Não deixo de agradecer a todos os meus amigos que estiveram presentes durante a minha vida de graduação e mestrado.

Agradeço ao Vinícius Morelli Cortes por ser um grande companheiro desde o primeiro dia de aula da graduação, enfrentando juntos diversos desafios acadêmicos ao longo dos semestres.

Ao André Ottenbreit Maschio Rodrigues por sempre me indicar diversos animes e mangás, e sempre me convidar para o karaokê para divertir e tirar o estresse.

Aos membros do Laboratório de Otimização, Combinatória e Algoritmos do CCSL, por sempre me convidar para estudar artigos científicos e para assistir diversas palestras.

Aos membros do NUMEC, por terem equipamentos, computadores e um lugar aconchegante para estudar e pesquisar.

Aos membros do grupo Grafões, que sempre tem novidades para obtermos novos conhecimentos tanto em teoria dos Grafos quanto em LaTeX.

Agradeço aos meus pais, Julio Kojiro Endo e Maria Angélica Kinoshita Endo, que sempre cuidam de mim com muito amor, me guiando para o caminho do sucesso; e à minha irmã, Lucy Yuko Endo, que, apesar de ser mais nova, me serve de exemplo.

A Júlia Kyomi Yabiku, agradeço por sempre cuidar de mim, e sempre estar ao meu lado durante os momentos difíceis de cada etapa do meu mestrado.

Agradeço também ao $\mathrm{CNPq}$ pelo financiamento, que me permitiu dedicação integral ao estudo da Matemática. 


\section{Resumo}

ENDO, E. O. Aproximação da Norma de Corte via Desigualdade de Grothendieck. 2014. 64 f. Dissertação (Mestrado) - Instituto de Matemática e Estatística, Universidade de São Paulo, São Paulo, 2014.

Neste trabalho, objetivamos apresentar o Teorema de Alon a Naor, o qual afirma que existe um algoritmo de aproximação para a norma de corte de uma matriz qualquer, sendo que a garantia de desempenho desse algoritmo é a inversa da constante de Grothendieck.

Introduzimos a norma de corte de uma matriz e exibimos algumas de suas propriedades. Uma delas é que a norma de corte é equivalente a uma outra norma, que é valor ótimo de um programa inteiro quadrático que pode ser relaxado por um programa semidefinido.

Além do Teorema de Alon e Naor, construímos mais dois algoritmos de aproximação para a norma de corte. Ambos possuem garantia de desempenho inferior que a do Teorema de Alon e Naor, porém as técnicas que foram utilizadas para obter tais algoritmos são interessantes.

Enunciamos a Desigualdade e Grothendieck reformulada por Lindenstrauss e Pełcýnski e mostramos uma cota superior para a constante de Grothendieck que se baseia no Argumento de Krivine.

Finalmente, apresentamos três aplicações do Teorema de Alon e Naor: em corte máximo de um grafo; na versão algorítmica do Lema da Regularidade de Szemerédi; e no Teorema de Frieze e Kannan.

Palavras-chave: Norma de Corte, Desigualdade de Grothendieck, Algoritmos de Aproximação, programa semidefinido. 


\section{Abstract}

ENDO, E. O. Approximation of the Cut-norm via Grothendieck's Inequality. 2014. $64 \mathrm{f}$. Thesis (Master) - Instituto de Matemática e Estatística, Universidade de São Paulo, São Paulo, 2014.

In this work, our objective is to present Alon and Naor's Theorem, which states that exist an approximation algorithm for cut-norm of any matrix, and that the aproximation guarantee of this algorithm is the inverse of the Grothendieck's constant.

We introduce the cut-norm of a matrix and we show some of its properties. One is that the cut-norm is equivalent of some other norm which is optimum value of quadratic integer program which can be relaxed for a semidefinite program.

Beyond Alon and Naor's Theorem, we construct two more approximation algorithm for cutnorm. The approximation guarantee of both is inferior than the Alon and Naor's Theorem, but the techniques for obtain such algorithms is interesting.

We show Grothendieck's Inequality reformulated by Lindenstrauss e Pełcýnski and we show an upper bound for the Grothendieck's constant which is based on Krivine's Argument.

Furthermore, we show three applications of Alon and Naor's Theorem: Maximum cut of a graph, algorithmic version of Szemerédi Regularity Lemma, and Frieze and Kannan's Theorem.

Keywords: Cut-norm, Grothendieck's Inequality, Approximation Algorithm, Semidefinite Program. 


\section{Sumário}

$\begin{array}{ll}\text { Lista de Símbolos } & \text { ix }\end{array}$

1 Introdução $\quad 1$

1.1 Outras aplicações . . . . . . . . . . . . . . . . . . . 2

1.2 Apresentação deste trabalho . . . . . . . . . . . . . . . . 2

2 Conceitos Básicos $\quad 3$

2.1 Matriz Positiva Semidefinida . . . . . . . . . . . . . . . . . . . . 3

2.2 Método da Eliminação Gaussiana . . . . . . . . . . . . . . . . . . . . . 6

2.3 Programação Semidefinida . . . . . . . . . . . . . . . . . . . . . 8

2.4 Método do elipsóide . . . . . . . . . . . . . . . . . . . . . . 10

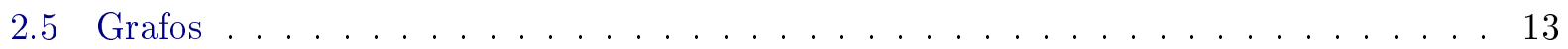

2.6 Complexidade Computacional . . . . . . . . . . . . . . . . . 14

2.7 Algoritmo de Aproximação . . . . . . . . . . . . . . . . . . . . . . 16

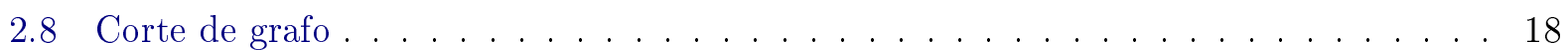

3 Norma de Corte $\quad \mathbf{2 1}$

3.1 A norma de corte e suas propriedades . . . . . . . . . . . . . 21

3.2 Aproximação determinística da norma de corte . . . . . . . . . . . . . 25

3.3 Melhorando a garantia de desempenho . . . . . . . . . . . . . . . . . . 30

4 Desigualdade de Grothendieck 33

4.1 Argumento de Krivine . . . . . . . . . . . . . . . . . . . . . . . 33

4.2 Aproximação da norma de corte via Desigualdade de Grothendieck . . . . . . . . . . 37

5 Aplicações da Norma de Corte $\quad 41$

5.1 Relação entre norma de corte e corte de grafo . . . . . . . . . . . . . . . . . . . . 41

5.2 Lema da Regularidade de Szemerédi . . . . . . . . . . . . . . . . . . . . . . . . . . . . . . . . . 4

5.3 Decomposição Matricial de Frieze e Kannan . . . . . . . . . . . . . . . . . . . . . . 46

$\begin{array}{ll}\text { Referências Bibliográficas } & 49\end{array}$ 
viii SUMÁRIO 


\section{Lista de Símbolos}

$[n]=\{1, \ldots, n\}$

$\mathbb{Q}:$ Conjunto dos números racionais

$\mathbb{R}:$ Conjunto dos números reais

$\mathbb{R}_{\geq 0}$ : Conjunto dos números reais não-negativos

$S^{n}$ : Esfera de raio unitário em $\mathbb{R}^{n}$ com centro na origem 


\section{Capítulo 1}

\section{Introdução}

A norma de corte de uma matriz real $A=\left(a_{i j}\right)$ do tipo $m \times n$, denotada por $\|A\|_{c u t}$, é o maior valor de $\left|\sum_{i \in I, j \in J} a_{i j}\right|$ sobre todo $I \subseteq[m]$ e $J \subseteq[n]$.

Em 2006, Alon e Naor [AN06] mostraram que o problema de aproximar a norma de corte de uma matriz é MAX SNP-difícil, mostrando que, para todo grafo $G$, existe uma matriz $A$ tal que a norma de corte de $A$ é igual ao corte máximo de $G$. Ademais, forneceram um algoritmo de aproximação em tempo polinomial para a norma de corte. Esse algoritmo encontra, para toda matriz $A=\left(a_{i j}\right)$ do tipo $m \times n$, dois subconjuntos $S_{0} \subset[m]$ e $T_{0} \subset[n]$ tais que

$$
\left|\sum_{\substack{i \in S_{0} \\ j \in T_{0}}} a_{i j}\right| \geq \rho\|A\|_{c u t},
$$

onde $\rho>0.56$ é uma constante que não depende da matriz $A$.

Frieze e Kannan em [FK96] introduziram a norma de corte para obter uma versão mais fraca do Lema de Regularidade de Szemerédi [Sze78]. Eles estavam interessados em procurar tal versão desse lema pelo fato de Gowers ter provado [Gow97] que, em alguns casos, o número de partes da partição obtida no Lema da Regularidade de Szemerédi cresce na ordem de uma torre de expoentes de tamanho polinomial. Portanto, se fez necessário desenvolver versões mais fracas do lema de forma que um computador possa obter as constantes, que não podem ser muito grandes.

Recordemos que o Lema da Regularidade de Szemerédi é um lema fundamental na teoria dos grafos. Foi enunciado e provado por Szemerédi com o objetivo de provar uma Conjectura de Erdôs e Turán [ET36] que afirma que existem progressões aritméticas de comprimento arbitrariamente grande em subconjuntos de densidade superior positiva de inteiros. O Lema da Regularidade de Szemerédi afirma que, de modo geral, o conjunto dos vértices de qualquer grafo pode ser particionado em subconjuntos de vértices $V_{1}, \ldots, V_{k}$ tais que quase todos seus grafos bipartidos induzidos $\left(V_{i}, V_{j}\right)$ se comportam como grafos aleatórios (veja Seção 5.2).

O algoritmo de aproximação de Alon e Naor se baseia na Desigualdade de Grothendieck. Essa desigualdade, provada por Grothendieck em [Gro53], é uma poderosa ferramenta de análise funcional que possui diversas aplicações em várias áreas, como em $C^{*}$-Álgebras [Pis86], Mecânica Quântica [Tsi85], Teoria da Informação Quântica $\left[\mathrm{PGWP}^{+} 08\right.$ ] e Ciência da Computação [AN06]. Podemos mencionar informalmente que a Desigualdade de Grothendieck é uma relação não-trivial entre espaços de Hilbert e dois espaços de Banach fundamentais $L_{\infty}$ e $L_{1}$. O algoritmo de aproximação de Alon e Naor possui inúmeras aplicações. Uma delas é a melhoria do algoritmo de Frieze e Kannan. Enquanto o algoritmo de Frieze e Kannan é relevante somente para instâncias "densas", o algoritmo de Alon e Naor pode ser aplicado para qualquer matriz. 


\section{$1.1 \quad$ Outras aplicações}

Veremos na Seção 3.1 que a norma de corte é equivalente à norma

$$
\|A\|_{\infty \rightarrow 1}=\max _{\varepsilon_{i}, \delta_{j} \in\{-1,1\}} \sum_{i=1}^{m} \sum_{j=1}^{n} a_{i j} \varepsilon_{i} \delta_{j},
$$

que possui um significado em Mecânica Estatística. Podemos utilizá-la para encontrar um estado de energia mínima ${ }^{1}$ do modelo de Ising, um modelo ferromagnético da Mecânica Estatística. O trabalho nessa direção pode ser encontrado em [LP09].

Uma outra aplicação do Teorema de Alon e Naor aparece na Biologia Computacional Molecular. Ao tentar identificar grupo de genes com comportamento correlacionado estatisticamente significativo em diversos experimentos, deseja-se resolver um certo problema de biclustering. Veja [TSKS04] e [TSS02] para mais informações. O problema computacional aqui é encontrar eficientemente, dada uma matriz cujas entradas são logaritmos de certas probabilidades, uma submatriz com maior valor (aproximadamente) da soma total das entradas.

Há diversas outras aplicações da Desigualdade de Grothendieck na Teoria da Computação, como em Otimização [Nes98] [NRT99] [Meg01], Machine Learning [KN09] [KN13], Teoria de Sistemas [BTN02], Complexidade de Comunicação [LS07], Teoria da Informação Quântica [Tsi85] [RT10], e Complexidade Computacional [KO09] [KN08] [RS09].

\subsection{Apresentação deste trabalho}

O objetivo deste trabalho é apresentar o Teorema de Alon e Naor e expor algumas de suas aplicações.

No Capítulo 2 introduzimos as notações e os conceitos básicos que serão utilizados ao longo do trabalho. Faremos um breve resumo sobre matrizes positivas semidefinidas, noções básicas de programação linear, de algoritmos de aproximação e de grafos.

No Capítulo 3 definimos a norma de corte e apresentaremos algumas de suas propriedades. Apresentaremos dois resultados de Grötschel, Lovász e Schrijver [GLS81] sobre otimização convexa. Aplicamos um dos resultados em programas semidefinidos para obter um algoritmo em tempo polinomial que calcula aproximadamente o valor ótimo do programa semidefinido. Apresentamos dois algoritmos de aproximação em tempo polinomial que produzem, para toda matriz $A=\left(a_{i j}\right)$ do tipo $m \times n$, inteiros $\varepsilon_{i}, \delta_{j} \in\{-1,1\}$ com $1 \leq i \leq m$ e $1 \leq j \leq n$ tais que $\sum_{i=1}^{m} \sum_{j=1}^{n} a_{i j} \varepsilon_{i} \delta_{j} \geq$ $\rho\|A\|_{\infty \rightarrow 1}$. Na Seção 3.2 apresentamos o algoritmo com $\rho=0.03$, e na Seção 3.3, o algoritmo com $\rho=0.27$.

No Capítulo 4 apresentamos o objetivo principal: provar o Teorema de Alon e Naor [AN06] que afirma que existe um algoritmo em tempo polinomial tal que, para toda matriz $A=\left(a_{i j}\right)$ do tipo $m \times n$, encontra subconjuntos $S_{0} \subseteq[m]$ e $T_{0} \subseteq[n]$ tais que $\left|\sum_{i \in S_{0}, j \in T_{0}} a_{i j}\right| \geq K_{G}^{-1}\|A\|_{c u t}$, onde $K_{G}$ é a constante de Grothendieck. Apresentamos também a Desigualdade de Grothendieck e sua a prova feita em [AN06].

No Capítulo 5, apresentamos aplicações do Teorema de Alon e Naor. Serão três aplicações: problema de corte máximo de um grafo; Lema da Regularidade de Szemerédi; e Teorema de Frieze e Kannan. Na Seção 5.1 demonstramos que o problema de calcular a norma de corte de um grafo arbitrário é MAX SNP-difícil. As principais fontes para esses capítulos foram os artigos [KRT03], [ADL ${ }^{+}$94], [FK96], [KN12], [DKM $\left.{ }^{+} 12\right]$.

\footnotetext{
${ }^{1} \mathrm{O}$ termo energia mínima é usualmente denominado ground state na literatura acadêmica.
} 


\section{Capítulo 2}

\section{Conceitos Básicos}

O objetivo deste capítulo é revisar alguns conceitos básicos e fixar notações. Vários dos conceitos apresentados em cada seção serão introdutórios, sem se preocupar com formalismos.

Na Seção 2.1 recordamos propriedades de matrizes positivas semidefinidas, e mostramos algumas de suas equivalências. Na Seção 2.2 recordamos brevemente o método da eliminação Gaussiana, e obtemos um algoritmo em tempo polinomial que decide se uma matriz é positiva semidefinida a partir do método. Na Seção 2.3 introduzimos programação semidefinida e apresentamos alguns exemplos. Na Seção 2.4 apresentamos dois resultados de otimização convexa que afirmam que, de modo geral, existe um método em tempo polinomial que calcula, com precisão arbitrariamente boa, o maior valor de um funcional linear sobre um conjunto convexo. Aplicaremos um dos resultados para obter tal algoritmo em programas semidefinidos. Na Seção 2.5 definiremos o conceito de grafo, grafo orientado e algumas notações. Na Seção 2.6 introduzimos o conceito de complexidade computacional, e apresentamos exemplos para cada classe de complexidade exibida na seção. Na Seção 2.7 definimos algoritmo de aproximação, o esquema de aproximação em tempo polinomial e a classe de complexidade MAX SNP-difícil. Na Seção 2.8 apresentamo o problema de corte de um grafo e alguns de seus resultados.

\subsection{Matriz Positiva Semidefinida}

Trabalhamos apenas com matrizes reais. Os elementos de $\mathbb{R}^{n}$ são vetores-coluna, e assumimos que todas as matrizes quadradas são do tipo $n \times n$, salvo algumas exceções, e nesse caso o tipo será exibido. Denotemos por $M(m, n)$ o conjunto das matrizes do tipo $m \times n$.

Seja $A$ uma matriz quadrada. A inversa de $A$ é uma matriz denotada por $A^{-1}$ tal que $A^{-1} A=$ $A A^{-1}=I$, onde $I$ é a matriz identidade. Um autovetor de $A$ é um vetor $v$ em $\mathbb{R}^{n}$ tal que $A v$ é paralelo ao vetor $v$, em outras palavras, existe um real $\lambda$ tal que $A v=\lambda v$. O número $\lambda$ é chamado de autovalor de $A$ associado ao autovetor $v$. Claramente $\lambda$ é autovalor se, e somente se, a matriz $A-\lambda I$ é não-singular, isto é, não possui inversa, ou equivalentemente, satisfaz $\operatorname{det}(A-\lambda I)=0$. Note que o determinante de $A-\lambda I$ é um polinômio de grau $n$, logo a matriz $A$ possui no máximo $n$ autovalores, incluindo as multiplicidades.

O traço da matriz quadrada $A=\left(a_{i j}\right)$ é definido por

$$
\operatorname{tr}(A)=\sum_{i=1}^{n} a_{i i}
$$

O produto interno usual de duas matrizes quadradas $A=\left(a_{i j}\right), B=\left(b_{i j}\right)$ é definido por

$$
\langle A, B\rangle=\operatorname{tr}\left(A^{t} B\right)=\sum_{i=1}^{n} \sum_{j=1}^{n} a_{i j} b_{i j}
$$

onde $A^{t}$ é a transposta da matriz $A$. A norma $\|A\|_{F}=\sqrt{\langle A, A\rangle}$ é chamada de norma de Frobenius. 
Uma matriz $A$ é dita ortogonal se os vetores-colunas formam uma base ortonormal de $\mathbb{R}^{n}$, e é dita simétrica se $A=A^{t}$. Claramente, toda matriz simétrica é quadrada. Denotemos por $\mathcal{S}^{n}$ o conjunto de todas as matrizes simétricas do tipo $n \times n$. Uma matriz $A=\left(a_{i j}\right)$ é diagonal se $a_{i j}=0$ para todo $i \neq j$.

Se a matriz $A$ é simétrica, então existem $n$ autovalores de $A$, contando com as multiplicidades. Um dos principais resultados sobre autovalores e autovetores é conhecido por Teorema da Decomposição Espectral.

Teorema 1. Toda matriz simétrica A pode ser decomposta como

$$
A=\sum_{i=1}^{n} \lambda_{i} v_{i} v_{i}^{t},
$$

onde $\lambda_{1}, \ldots, \lambda_{n} \in \mathbb{R}$ são os autovalores de $A$, e $v_{1}, \ldots, v_{n} \in \mathbb{R}^{n}$ são os autovetores de $A$ correspondentes que formam uma base ortonormal em $\mathbb{R}^{n}$. Em outras palavras, toda matriz simétrica $A$ pode ser escrita como $W^{t} D W$, onde $W$ é uma matriz ortogonal cujas colunas são autovetores de $A$ e $D$ é uma matriz diagonal cujas entradas da diagonal são autovalores de A.

Podemos ver a prova do Teorema 1 em [HK71].

Um menor simétrico de uma matriz simétrica $A$ é uma matriz $B$ obtida da seguinte forma: Considere $[n]$ o conjunto dos índices das linhas e das colunas de $A$. Existe $R \subseteq[n]$ tais que $B=$ $\left(b_{l k}\right)=\left(a_{i_{l} j_{k}}\right)$, onde $i_{j}, j_{k} \in R$. Um menor simétrico é dito menor principal se $R=\{1, \ldots, t\}$ para algum $1 \leq t \leq n$. Uma matriz simétrica $A$ é dita positiva semidefinida se todo autovalor de $A$ é não-negativo. Essa propriedade será denotada por $A \succeq 0$. Denotemos por $\mathcal{S}_{\succeq 0}^{n}$ o conjunto de todas as matrizes positivas semidefinidas do tipo $n \times n$.

A proposição abaixo apresenta caracterizações das matrizes positivas semidefinidas.

Proposição 2. Seja A uma matriz simétrica. São equivalentes:

(i) A matriz A é positiva semidefinida;

(ii) Existe uma matriz quadrada $U$ tal que $A=U^{t} U$;

(iii) Para todo $x \in \mathbb{R}^{n}$, o valor $x^{t} A x$ é não-negativo;

(iv) O determinante de todo menor simétrico da matriz A é não-negativo.

Demonstração. Mostremos que (i) implica (ii). Sejam $W$ uma matriz ortogonal cujas colunas são autovetores de $A$ e $D$ a matriz diagonal cujas entradas da diagonal são autovalores de $A$. Temos que $A=W^{t} D W$. Como as entradas da diagonal de $D$ são os autovalores de $A$ e são não-negativos, existe uma matriz diagonal $C$ tal que $D=C^{2}$. Claramente $C$ é simétrica. Assim

$$
A=W^{t} D W=W^{t} C C W=(C W)^{t}(C W),
$$

como queríamos.

Mostremos que (ii) implica (iii). Sejam $x \in \mathbb{R}^{n}$ um vetor qualquer e $U$ uma matriz satisfazendo $A=U^{t} U$. Temos

$$
x^{t} A x=x^{t}\left(U^{t} U\right) x=(U x)^{t}(U x) \geq 0,
$$

como queríamos.

Mostremos que (iii) implica (i). Sejam $\lambda$ um autovalor de $A$ e $v$ um autovetor de $A$ associado ao autovalor $\lambda$. Temos

$$
0 \leq v^{t} A v=v(\lambda v)=\lambda v^{t} v .
$$

Como $v^{t} v>0$, concluímos que $\lambda \geq 0$.

Mostremos que (ii) implica (iv). Sejam $R \subseteq[n]$ e $B$ o menor simétrico de $A$ do tipo $R \times R$. Sejam $U$ tal que $A=U^{t} U$, e $V$ a matriz obtida de $U$ tomando somente as colunas em $R$. Temos

$$
\operatorname{det}(B)=\operatorname{det}\left(V^{t} V\right)=\operatorname{det}(V)^{2} \geq 0 .
$$


Mostremos que (iv) implica (i) pela contrapositiva. Suponhamos que existe um autovetor $v$ de $A$ tal que o autovalor associado é $\lambda<0$.

Se $A$ possui somente um autovalor negativo, então $\operatorname{det}(A)=\operatorname{det}\left(W^{t} D W\right)=\operatorname{det}(D)<0$, onde $W$ é a matriz ortogonal cujas colunas são autovetores de $A$ e $D$ é a matriz diagonal cujas entradas da diagonal são autovalores de $A$. Neste caso não há nada para fazer. Suponha que existe um autovetor $w$ linearmente independente de $v$ tal que o autovetor associado é $\mu<0$. Considere um real $s \in \mathbb{R}$ tal que o vetor $u=v+s w$ se anula em alguma componente, digamos, na $i$-ésima. Seja $A^{\prime}$ a matriz obtida de $A$ removendo a $i$-ésima linha e a $i$-ésima coluna, e seja $u^{\prime}$ o vetor obtido de $u$ removendo a $i$-ésima componente. Temos

$$
\left(u^{\prime}\right)^{t} A^{\prime} u^{\prime}=u^{t} A u=(v+s w)^{t} A(v+s w)=\left(v^{t}+s w^{t}\right)(\lambda v+s \mu w)=\lambda+s^{2} \mu<0 .
$$

Como o item $(i)$ é equivalente ao item (iii), temos que $A^{\prime}$ não é positiva semidefinida. Aplicando o procedimento acima indutivamente, obtemos uma matriz com somente um autovalor negativo, e logo seu determinante é negativo, contradizendo o item $(i v)$.

Um conjunto $K$ de $\mathbb{R}^{n}$ é dito cone convexo se é fechado por combinações lineares não-negativas: Para todo $\alpha, \beta$ reais não-negativos e para todo $x, y \in K$, temos $\alpha x+\beta y \in K$. O cone convexo gerado pelo conjunto de vetores $A$ em $\mathbb{R}^{n}$ é dado pelo menor cone convexo que contém $A$, denotado por cone $(A)$. Ademais,

$$
\operatorname{cone}(A)=\left\{\sum_{i=1}^{N} \alpha_{i} x_{i}: N \in \mathbb{N}, x_{1}, \ldots, x_{N} \in A, \alpha_{1}, \ldots, \alpha_{N} \in \mathbb{R}_{\geq 0}\right\} .
$$

Note que a soma de matrizes positivas semidefinidas, e o produto de um escalar positivo com uma matriz positiva semidefinida, é uma matriz positiva semidefinida. Portanto o conjunto das matrizes positivas semidefinidas é um cone convexo.

Pela Propriedade (iii) da Proposição 2, temos que para todo $x \in \mathbb{R}^{n}$ a matriz $x x^{t}$ é positiva semidefinida. De fato, para todo vetor $y \in \mathbb{R}^{n}$, temos

$$
y^{t} x x^{t} y=\left(x^{t} y\right)^{t}\left(x^{t} y\right) \geq 0 .
$$

Note que, pelo Teorema 1 , o conjunto $\mathcal{S}_{\succeq 0}^{n}$ pode ser escrito como

$$
\mathcal{S}_{\succeq 0}^{n}=\operatorname{cone}\left\{x x^{t}: x \in \mathbb{R}^{n}\right\}
$$

isto é, $\mathcal{S}_{\succ 0}^{n}$ é um cone convexo gerado pelas matrizes de posto um.

Matrizes que estão contidas no interior de $\mathcal{S}_{\succ 0}^{n}$ são chamadas de matrizes positivas definidas. $\mathrm{O}$ seguinte resultado apresenta diversas caracterizações de matrizes positivas definidas.

Proposição 3. Seja A uma matriz simétrica do tipo $n \times n$. São equivalentes:

(i) A matriz A é positiva definida;

(ii) Existe uma matriz quadrada $U$ de posto $n$ tal que $A=U^{t} U$;

(iii) Para todo $x \in \mathbb{R}^{n} \backslash\{0\}$, o valor $x^{t} A x$ é positivo;

(iv) O determinante de todo menor simétrico da matriz A é positivo;

(v) Todos os autovalores de A são positivos.

Podemos ver a prova da Proposição 3 em [HJ13].

Para mais informações sobre teoria das matrizes e álgebra linear, recomendamos os livros [HK71], [Lan87] e [HJ13]. 


\subsection{Método da Eliminação Gaussiana}

Dada uma matriz simétrica, uma questão interessante de levantar é se podemos obter um algoritmo em tempo polinomial que decide se tal matriz é ou não positiva semidefinida, e que produz um certificado se a matriz não for positiva semidefinida, por exemplo, produz um vetor $x$ tal que $x^{t} A x<0$. Existem diversos algoritmos que resolvem essa questão, e podemos vê-los em [GvL13]. Apresentamos aqui o algoritmo que se baseia no método da eliminação Gaussiana, um método que escalona sistemas lineares.

Todas as matrizes desta seção tem entradas racionais, esta hipótese é necessária para cálculos em computadores. Uma matriz $A=\left(a_{i j}\right)$ é dita triangular inferior se $a_{i j}=0$ para todo $i<j$; e é dita triangular superior se $a_{i j}=0$ para todo $i>j$. Uma matriz é dita unitária se a diagonal principal tem todas as entradas iguais a um.

Definição 4. Uma operação elementar é uma função de $M(n, n)$ em $M(n, n)$ que, aplicada em uma matriz quadrada, satisfaz somente uma das seguintes operaçôes:

(1) troca duas linhas da matriz;

(2) multiplica uma linha por um número diferente de zero;

(3) substitui uma linha pela sua soma com outra linha da matriz multiplicada por um número.

Uma matriz quadrada é dita elementar se é obtida da matriz identidade aplicando uma das operações elementares. Uma matriz elementar é do tipo $(i)$ se é obtida da matriz identidade aplicando a operação $(i)$.

O método da eliminação Gaussiana é um método que, usando somente a operação elementar (3), encontra uma solução para um sistema linear $A x=b$, ou conclui que $A$ é singular. $\mathrm{O}$ algoritmo desse método, no $k$-ésimo passo, "transforma" a matriz $A$ em uma matriz da forma

$$
B=\left(\begin{array}{cc}
B_{11} & B_{12} \\
0 & B_{22}
\end{array}\right),
$$

onde $B_{11}$ é uma matriz triangular inferior unitária do tipo $(k-1) \times(k-1)$. A "transformação" consiste em multiplicar à esquerda as matrizes elementares do tipo (3) com a matriz $A$.

O seguinte resultado afirma que podemos decompor uma matriz como produto de duas matrizes específicas.

Teorema 5 (Teorema da Decomposição LU). Seja A uma matriz quadrada cujos menores principais são todos não-singulares. Então A pode ser decomposta unicamente como

$$
A=L U,
$$

onde $L$ é uma matriz unitária triangular inferior, e U é uma matriz triangular superior.

O algoritmo do método da eliminação Gaussiana produz as matrizes $L$ e $U$ da decomposição da matriz $A$ da seguinte forma: A matriz $U$ é a matriz $A$ triangularizada e $L^{-1}$ é o produto das matrizes elementares usadas durante o método. Podemos ver a prova do Teorema 5 em [GvL13].

Segundo [GvL13], podemos usar o Teorema 5 para resolver um sistema linear da seguinte forma: Seja $A x=b$ um sistema linear. Para resolvê-lo, reduzimos na forma $U x=y$, onde $U$ é uma matriz triangular superior, e $y$ é uma solução do sistema $L y=b$, onde $L$ é uma matriz unitária triangular inferior. Assim $L U x=b$, e portanto $A x=b$.

Porém, estamos interessados no método da eliminação Gaussiana com pivô que, usando as operações elementares (1) e (3), encontra uma solução para um sistema linear $A x=b$, ou conclui 
que $A$ é singular. Esse método consiste em transformar a matriz $A$ em uma matriz triangular superior, com diagonal não-nula. Os elementos dessa diagonal são chamados de pivô.

A estratégia de pivoteamento no algoritmo do método da eliminação Gaussiana com pivô é dada por:

1. No início do passo $k$ do método da eliminação Gaussiana, escolher para o pivô o elemento de maior módulo entre os valores $a_{i k}$, onde $k \leq i \leq n$;

2. Trocar as linhas $k$ e $i$ se necessário.

Suponhamos que no $k$-ésimo passo, a matriz $A$ se "transformou" na matriz $B$ dada em (2.1). Aplicamos a estratégia de pivoteamento, e procedemos o algoritmo como no método da eliminação Gaussiana.

Uma matriz de permutação é uma matriz quadrada que tem exatamente uma entrada igual a um em cada linha e cada coluna, e as entradas restantes são iguais a zero. Note que toda matriz de permutação é produto de matrizes elementares do tipo (1). Não é difícil ver que toda matriz de permutação é ortogonal.

O seguinte resultado afirma que podemos decompor uma matriz como produto de três matrizes específicas.

Teorema 6. Dada uma matriz quadrada A, a eliminação Gaussiana com pivô produz uma matriz unitária triangular inferior $L$, uma matriz triangular superior $U$, e uma matriz de permutação $P$ tais que

$$
A=P^{t} L U \text {. }
$$

Podemos ver a prova do Teorema 6 em [GvL13]. O processo para obter as matrizes $L, U$ e $P$ consome tempo polinomial e usa o método da eliminação Gaussiana com pivô. Note que $P$ e $L$ são não-singulares.

Considere o problema de resolver $A x=b$ quando $A$ é uma matriz simétrica. Para preservar a simetria, quando trocamos as linhas de $A$ temos que trocar também as colunas de $A$. Ademais, quando substituímos uma linha pela sua soma com outra linha da matriz multiplicada por um número, devemos fazer o mesmo para a coluna. Isso mostra que a eliminação Gaussiana com pivô é da forma

$$
A=P^{t} L U L^{t} P
$$

O intuito de decompor a matriz $A$ preservando a simetria é para garantir um resultado que caracteriza quando $A$ é positiva semidefinida.

Proposição 7. Seja A uma matriz simétrica decomposta como em (2.2). Então A é positiva semidefinida se e somente se $U$ é positiva semidefinida.

Demonstração. Suponhamos que $A$ é positiva semidefinida. Como $P$ e $L$ são não-singulares, temos que $L^{t} P$ é não-singular. Logo para todo $x \in \mathbb{R}^{n}$ existe $y \in \mathbb{R}^{n}$ tal que $x=L^{t} P y$. Portanto

$$
x^{t} U x=\left(L^{t} P y\right)^{t} U\left(L^{t} P y\right)=y^{t}\left(P^{t} L U L^{t} P\right) y=y^{t} A y \geq 0
$$

para todo $x \in \mathbb{R}^{n}$.

Agora suponhamos que $U$ é positiva semidefinida. Seja $x \in \mathbb{R}^{n}$ um vetor qualquer. Temos

$$
x^{t} A x=x^{t}\left(P^{t} L U L^{t} P\right) x=\left(L^{t} P x\right)^{t} U\left(L^{t} P x\right) \geq 0,
$$

como queríamos.

Infelizmente, por estarmos operando simultaneamente com as linhas e as colunas para decompor uma matriz simétrica $A$, nem sempre podemos aplicar o algoritmo do Teorema 6 até o fim, mesmo 
no caso não-singular. Por exemplo, considere a matriz

$$
A=\left(\begin{array}{ll}
0 & 1 \\
1 & 0
\end{array}\right) .
$$

Temos que para toda matriz de permutação $P$, temos $P^{t} A P=A$. Logo nunca conseguimos transformar a matriz $A$ de forma que tenha um elemento na diagonal não-nula. Mas mesmo com esse problema, podemos aproveitar o algoritmo do Teorema 6 para decidir se uma matriz $A$ é ou não positiva semidefinida, e se não for, podemos encontrar uma vetor $x$ tal que $x^{t} A x<0$.

Seja $A$ uma matriz simétrica. Pelo Teorema 6 e pela observação acima, podemos aplicar o algoritmo do método da eliminação Gaussiana com pivô. Suponhamos que no $k$-ésimo passo da decomposição decompomos a matriz $A$ da forma $A=P^{t} L U L^{t} P$.

Se, no $(k+1)$-ésimo passo, obtemos uma entrada da diagonal negativa, temos que a matriz $U$ não é positiva semidefinida, e pela Proposição 7 , temos que $A$ não é positiva semidefinida. Seja $i$ a linha que contém a entrada da diagonal negativa e $x=\left(L^{t} P\right)^{-1} e_{i}$, onde $e_{i}$ é o vetor com entrada igual a um na $i$-ésima componente, e igual a zero na restante. Temos $x^{t} A x<0$.

Suponhamos agora que, no $(k+1)$-ésimo passo, obtemos uma entrada da diagonal nula e a linha que a contém possui um elemento não-nulo, então a matriz $U$ não é positiva semidefinida. De fato, escrevendo $U=\left(u_{i j}\right)$, seja $i$ a linha que contém a entrada da diagonal zero, isto é, $u_{i i}=0$, e $j$ a coluna tal que $u_{i j} \neq 0$. Seja $B$ o menor simétrico da matriz $U$ dado por

$$
B=\left(\begin{array}{ll}
u_{i i} & u_{i j} \\
u_{i j} & u_{j j}
\end{array}\right)
$$

Temos que o determinante de $B$ é igual a $\operatorname{det}(B)=-u_{i j}^{2}<0$. Pelo item (iv) da Proposição 2, concluímos que $U$ não é positiva semidefinida. Portanto $A$ não é positiva semidefinida. Se $u_{j j} \neq 0$, tome $x=\left(L^{t} P\right)^{-1}\left(u_{j j} e_{i}-u_{i j} e_{j}\right)$, assim $x^{t} A x=-u_{i j}^{2} u_{j j} \leq 0$. Se $u_{j j}=0$, tome $x=\left(L^{t} P\right)^{-1}\left(e_{i}+\right.$ $\left.u_{i j} e_{j}\right), \operatorname{assim} x^{t} A x=-2 u_{i j}^{2} \leq 0$.

Se terminarmos o processo da eliminação Gaussiana com pivô, concluímos que a matriz $A$ é positiva semidefinida. Obtemos então o seguinte resultado.

Teorema 8. Seja A uma matriz simétrica. Existe um algoritmo em tempo polinomial que decide se A é positiva semidefinida ou não. Caso seja, produz uma matriz unitária triangular inferior $L$, uma matriz triangular superior $U$, e uma matriz de permutação $P$ tais que

$$
A=P^{t} L U L^{t} P \text {. }
$$

Caso $A$ não seja positiva semidefinida, o algoritmo produz um vetor $x \in \mathbb{Q}^{n}$ tal que $x^{t} A x<0$.

\subsection{Programação Semidefinida}

Programação semidefinida ( $P S D$ ou $S D P^{1}$ ) é uma subárea de otimização convexa que se interessa por calcular o máximo ou o mínimo de uma função linear sobre a interseção do cone das matrizes positivas semidefinidas com um espaço afim. Uma característica interessante dos programas semidefinidos é que todos os problemas de programação linear podem ser expressos como um problema de programação semidefinida (Sugerimos o livro [Sch03] para saber mais sobre programação linear).

\footnotetext{
${ }^{1}$ Semidefinite Programming
} 
A programação semidefinida possui várias aplicações interessantes em diversas áreas, como em teoria de controle [Ba196], teoria da informação [Lov79] e problemas de satisfatibilidade [GW95], [dKVMW00]. Uma boa referência que apresenta aplicações de programação semidefinida em problemas de otimização combinatória é [Lov03].

Sejam $A_{1} \ldots, A_{n}, C$ matrizes simétricas do tipo $m \times m$ para algum $m$ inteiro positivo e $b=$ $\left(b_{j}\right)_{j \in[n]}$ um vetor em $\mathbb{R}^{n}$. Considere o subespaço afim de $\mathcal{S}^{n}$ dado por

$$
\mathcal{W}=\left\{X \in \mathcal{S}^{m}:\left\langle A_{1}, X\right\rangle=b_{1}, \ldots,\left\langle A_{n}, X\right\rangle=b_{n}\right\} .
$$

Um programa semidefinido é um problema de otimização da seguinte forma:

$$
\begin{aligned}
\sup & \langle C, X\rangle \\
\text { s.a. } & \left\langle A_{1}, X\right\rangle=b_{1} ; \\
& \vdots \\
& \left\langle A_{n}, X\right\rangle=b_{n} ; \\
& X \succeq 0 .
\end{aligned}
$$

Lembremos que $\langle A, B\rangle=\operatorname{tr}\left(A^{t} B\right)$.

Em outras palavras, um programa semidefinido maximiza a função objetivo $\langle C, X\rangle$ sobre a região viável

$$
\mathcal{F}=\mathcal{S}_{\succeq 0}^{m} \cap \mathcal{W}
$$

As matrizes que pertencem a $\mathcal{F}$ são chamadas de soluções viáveis, e as que atingem o supremo do programa (2) são chamadas de soluções ótimas. Uma solução viável $X \in \mathcal{F}$ é dita estritamente viável se $X$ é uma matriz positiva definida. O programa (2.7) é dito estritamente viável se admite pelo menos uma solução estritamente viável.

O valor ótimo de um programa semidefinido é dado por

$$
v_{\text {primal }}=\sup _{X \in \mathcal{F}}\langle C, X\rangle
$$

Note que $v_{\text {primal }} \in \mathbb{R} \cup\{ \pm \infty\}$, e $v_{\text {primal }}=-\infty$ se o problema (2.7) não é viável, isto é, o conjunto $\mathcal{F}$ é vazio; e $v_{\text {primal }}=+\infty$ se as soluções do problema é não-limitado. Note também que definimos os programas semidefinidos (2.7) como supremo, e não máximo, pois existem problemas que o supremo não é atingido sempre, como podemos ver no Exemplo 10 adiante.

Podemos trabalhar também com problemas de minimização. Nesse caso, o programa semidefinido será da forma

$$
\begin{aligned}
& \inf \langle C, X\rangle \\
& \text { s.a. }\left\langle A_{1}, X\right\rangle=b_{1} ; \\
& \quad \vdots \\
& \quad\left\langle A_{n}, X\right\rangle=b_{n} ; \\
& \quad X \succeq 0 .
\end{aligned}
$$

Note que podemos transformar o programa (2.4) em um programa (2.7) usando que $\inf \langle C, X\rangle=$ $-\sup \langle-C, X\rangle$.

Exemplo 9. Seja o problema PSD

$$
\begin{aligned}
& \min x \\
& \text { s.a }\left(\begin{array}{ll}
x & 1 \\
1 & 0
\end{array}\right) \succeq 0,
\end{aligned}
$$


onde $x \in \mathbb{R}$. Note que

$$
X=\left(\begin{array}{ll}
x & y \\
y & z
\end{array}\right), C=\left(\begin{array}{ll}
1 & 0 \\
0 & 0
\end{array}\right), A_{1}=\left(\begin{array}{ll}
0 & 1 \\
0 & 0
\end{array}\right), A_{2}=\left(\begin{array}{ll}
0 & 0 \\
0 & 1
\end{array}\right) .
$$

Também,

$$
\langle C, X\rangle=x, b=(1,0) .
$$

Como det $\left(\begin{array}{cc}x & 1 \\ 1 & 0\end{array}\right)=-1$ para todo $x \in \mathbb{R}$ e pelo item (iv) da Proposição 2, temos

$$
\mathcal{F}=\left\{x \in \mathbb{R}^{n}:\left(\begin{array}{cc}
x & 1 \\
1 & 0
\end{array}\right) \succeq 0\right\}=\emptyset
$$

Portanto, $v_{\text {primal }}=-\infty$.

Exemplo 10. Seja o problema PSD

$$
\begin{aligned}
& \min x_{1} \\
& \operatorname{s.a}\left(\begin{array}{ll}
x_{1} & 1 \\
1 & x_{2}
\end{array}\right) \succeq 0 .
\end{aligned}
$$

onde $x_{1}, x_{2} \in \mathbb{R}$. Note que

$$
X=\left(\begin{array}{ll}
x_{1} & y \\
y & x_{2}
\end{array}\right), C=\left(\begin{array}{ll}
1 & 0 \\
0 & 0
\end{array}\right), A_{1}=\left(\begin{array}{ll}
0 & 1 \\
0 & 0
\end{array}\right) .
$$

Também,

$$
\langle C, X\rangle=x_{1}, \quad b=(1) .
$$

Calculando os determinantes dos menores simétricos, temos $x_{1} \geq 0, x_{2} \geq 0$ e $x_{1} x_{2} \geq 1$. Logo, os valores possiveis da função objetivo são todos os números reais positivos. Assim v vrimal $=0$. Mas o infimo não é atingido. De fato, tomando $x_{1}=0$ a desigualdade $x_{1} x_{2} \geq 1$ não é satisfeita. Portanto, a solução ótima nem sempre é uma solução viável.

Para mais informações sobre programação semidefinida, recomendamos o livro [GM12] e os artigos [Lov03] e [LV12].

\subsection{Método do elipsóide}

Dado $c \in \mathbb{R}^{n}$, um programa convexo sobre um conjunto $K$ em $\mathbb{R}^{n}$ não-vazio, convexo e compacto, é um programa de otimização definido por

$$
\begin{aligned}
& \max c^{t} x \\
& \text { s.a. } x \in K .
\end{aligned}
$$

Considere dois problemas a seguir relacionados ao programa convexo acima: 
1. Problema de otimização forte: Dado um vetor $c \in \mathbb{R}^{n}$, encontre um vetor $x^{*} \in K$ que maximiza $c^{t} x$ em $K$.

2. Problema de separação forte: Dado um vetor $y \in \mathbb{R}^{n}$, decida se $y \in K$, ou encontre um hiperplano separando $y$ de $K$, isto é, encontre um vetor $a \in \mathbb{R}^{n}$ tal que $a^{t} y>\max \left\{a^{t} x: x \in\right.$ $K\}$.

Exemplo 11 (Programação linear). Dados $a_{i} \in \mathbb{R}^{n}$ e $b_{i} \in \mathbb{R}$, com $1 \leq i \leq m$, considere o seguinte programa de otimização:

$$
\begin{aligned}
& \max c^{t} x \\
& \text { s.a. } a_{i}^{t} x \leq b_{i}, \text { para todo } 1 \leq i \leq m .
\end{aligned}
$$

Temos que o conjunto $K=\left\{x \in \mathbb{R}^{n}: a_{i}^{t} x \leq b_{i}\right.$, para todo $\left.1 \leq i \leq m\right\}$ é convexo e compacto. A otimização acima é um problema de otimização forte. O problema de separação nesse caso é, dado $y \in \mathbb{R}^{n}$, decidir se y satisfaz todas as desigualdades na restrição do programa, ou se existe um hiperplano separando y de $K$. Caso não ocorra o primeiro caso, temos que existe $1 \leq i \leq m$ tal que $a_{i}^{t} y>b_{i}$, logo obtemos o vetor $a=a_{i}$ tal que $a^{t} y>b_{i} \geq \max \left\{a^{t} x: x \in K\right\}$.

Exemplo 12 (Programação semidefinida). Sejam $C, A_{1} \ldots, A_{m}$ matrizes em $\mathcal{S}^{n}$, e $b_{1} \ldots, b_{m}$ em $\mathbb{R}$. $O$ problema de otimização forte para $\mathcal{F}$ é

$$
\begin{aligned}
& \sup \langle C, X\rangle \\
& \text { s.a. }\left\langle A_{1}, X\right\rangle=b_{1} ; \\
& \quad \vdots \\
& \quad\left\langle A_{m}, X\right\rangle=b_{m} ; \\
& \quad X \succeq 0 .
\end{aligned}
$$

O problema de separação forte é, dada uma matriz simétrica $Y$, decidir se $Y$ é uma matriz positiva semidefinida em $\mathcal{F}$, ou existe um hiperplano separando $Y$ de $\mathcal{F}$. Temos duas formas de obter um hiperplano separador: Primeiro, se existe $1 \leq i \leq m$ tal que $\left\langle A_{i}, Y\right\rangle>b_{i}$, obtemos a matriz $A=A_{i}$ tal que $A^{t} Y>b_{i} \geq \max \left\{A^{t} X: X \in \mathcal{F}\right\}$. Segundo, caso $Y$ não é uma matriz positiva semidefinida mas $Y \in \mathcal{W}$, então, pela Proposição 2, existe $a \in \mathbb{R}^{n}$ tal que $a^{t} Y a<0$, portanto $\left\langle a a^{t}, Y\right\rangle<0 \leq \max \left\{\left\langle a a^{t}, X\right\rangle: X \in \mathcal{F}\right\}$.

Sabemos que, se o programa (2.5) for um programa linear, então existe um resultado fundamental de programação linear que afirma que o programa (2.5) tem uma solução ótima com entradas racionais. Porém para programas convexos em geral nem sempre é verdade. Temos que o valor ótimo do seguinte programa semidefinido

$$
\begin{aligned}
& \max x \\
& \text { s.a. }\left(\begin{array}{ll}
1 & x \\
x & 2
\end{array}\right) \succeq 0 .
\end{aligned}
$$

é $x=\sqrt{2}$. Como $\sqrt{2}$ é um número irracional, não há um algoritmo computacional que calcula explicitamente a solução do programa (2.8). Logo é natural querermos obter um algoritmo em tempo polinomial que calcula aproximadamente o valor ótimo do programa (2.5). Uma solução para esse problema é o método do elipsóide.

O método do elipsóide é um algoritmo de otimização sobre um conjunto convexo com interior não-vazio introduzido por Shor, Yudin e Nemirovskii (veja o capítulo 13 de Schrijver [Sch86]). Infelizmente, o método do elipsóide não é eficiente em geral na prática (veja a Seção 13.5 de Schrijver [Sch86]). Porém, um resultado de Grötschel, Lovász e Schrijver [GLS81] tornou-se uma ferramenta 
essencial em otimização usando o método do elipsóide, usado para provar a solubilidade de diversas classes de problemas de otimização, em particular, em problemas de otimização combinatória. Recomendamos o livro [GLS93] para mais detalhes sobre o método do elipsóide.

Vamos enunciar o resultado de Grötschel, Lovász e Schrijver [GLS81] e usá-lo para produzir um algoritmo em tempo polinomial que produz uma solução aproximada de programas semidefinidos.

Devido ao exemplo 2.8, necessitamos de uma versão mais fraca dos problemas de otimização e de separação.

Definição 13. Dado um convexo $K$ não-vazio e compacto em $\mathbb{R}^{n}$, definimos os seguintes problemas sobre $K$.

1. Problema de otimização fraca: Dado um vetor $c \in \mathbb{R}^{n}$ e um racional $\varepsilon>0$, encontre um vetor $\hat{x} \in \mathbb{Q}^{n}$ tal que $\mathrm{d}(\hat{x}, K) \leq \varepsilon$, e que para todo para todo $x \in K$, temos $c^{t} x \leq c^{t} \hat{x}+\varepsilon$.

2. Problema de separação fraca: Dado um vetor $y \in \mathbb{Q}^{n}$ e um racional $\delta>0$, obtenha uma das afirmaçôes: (1) conclua que $\mathrm{d}(y, K) \leq \delta$; ou (2) encontre um vetor $a \in \mathbb{Q}^{n}$ tal que $\|a\|_{\infty} \geq 1$ $e$, para todo $x \in K$, temos $a^{t} x \leq a^{t} y+\delta$.

Recordemos que, se $x=\left(x_{1}, \ldots, x_{n}\right)$, então $\|x\|_{\infty}=\max \left\{\left|x_{i}\right|: 1 \leq i \leq n\right\}$, e

$$
\mathrm{d}(x, K)=\inf \left\{\|x-y\|_{2}: y \in K\right\} .
$$

Necessitamos da restrição $\|a\|_{\infty} \geq 1$ no problema de separação fraca pois poderíamos tomar $a=0$, satisfazendo sempre a desigualdade $a^{t} x \leq a^{t} s+\delta$. Note que o problema de separação fraca ou certifica que o vetor é "quase" viável, ou seja, possui uma distância pequena do conjunto convexo, ou encontra um hiperplano que "quase" separa o vetor do conjunto convexo.

Exemplo 14. Sejam $C, A_{1} \ldots, A_{m}$ matrizes em $\mathcal{S}^{n}$, e $b_{1} \ldots, b_{m}$ em $\mathbb{R}$, e considere os conjuntos

$$
\begin{aligned}
\mathcal{W} & =\left\{X \in \mathcal{S}^{n}:\left\langle A_{1}, X\right\rangle=b_{1}, \ldots,\left\langle A_{m}, X\right\rangle=b_{m}\right\} ; \\
\mathcal{F} & =\mathcal{S}_{\succeq 0}^{n} \cap \mathcal{W} .
\end{aligned}
$$

O Método da Eliminaçẫo Gaussiana é um algoritmo para o problema de separação fraca para o conjunto $\mathcal{F}$. Note que $\mathcal{F}$ possui interior vazio, pois está contido no conjunto $\mathcal{W}$. Mas isso não é um problema, pois podemos restringir $\mathcal{F}$ para o espaço $\mathcal{W}$, e assim o nosso conjunto convexo terá interior não-vazio.

O Método da Eliminação Gaussiana decide, em tempo polinomial, se uma matriz $A$ é positiva semidefinida. Ademais, caso não for, encontramos um vetor $x$ tal que $x^{t} A x<0$. Note que $x^{t} A x=\left\langle x x^{t}, A\right\rangle$, logo obtemos um hiperplano separador que separa a matriz $A$ do conjunto $\mathcal{F}$. Segundo [Lov03], necessitamos do erro $\varepsilon$ por problemas numéricos ao utilizar o método da eliminação Gaussiana.

Um corpo convexo é uma quíntupla $\left(K, n, a_{0}, r, R\right)$, onde $n \geq 2, K \subseteq \mathbb{R}^{n}$ é um conjunto convexo, $a_{0} \in K$, e

$$
B\left(a_{0}, r\right) \subseteq K \subseteq B\left(a_{0}, R\right),
$$

onde $B(z, r)=\left\{x \in \mathbb{R}^{n}:\|x-z\|_{2} \leq r\right\}$ é a bola de centro $z$ e raio $r$. Como uma das hipóteses de $K$ para problemas de otimização é que $K$ seja limitado, dizer que $K$ está contido em uma bola é natural. Agora, supor que $K$ contém uma bola não é tão natural. Essa suposição diz que $K$ não pode ser imersa em um subespaço de $\mathbb{R}^{n}$ de dimensão menor que $n$, ou que pelo menos sabe-se de um subespaço afim que contém $K$.

Considere $\{0,1\}^{*}$ o conjunto de todas as sequências em $\{0,1\}$ de tamanho finito. Dado $\Pi \subseteq$ $\{0,1\}^{*}$, considere a classe de corpos convexos

$$
\mathcal{K}=\left\{K_{\sigma}: \sigma \in \Pi \text { e } K_{\sigma} \text { é um corpo convexo }\right\}
$$


A entrada do problema de otimização fraca é a upla $\left(\sigma, n, a_{0}, r, R, c, \varepsilon\right)$, onde $\sigma \in \Pi, K_{\sigma}=$ $\left(K, n, a_{0}, r, R\right), c \in \mathbb{Q}^{n}$, e $\varepsilon>0$. Um algoritmo para o problema de otimização fraca para a classe $\mathcal{K}$ é um algoritmo que recebe como entrada a upla acima e resolve o problema de otimização fraca para o corpo convexo $K_{\sigma}$. Um algoritmo é em tempo polinomial se o número de operações para obter a saída do algoritmo é polinomial (que depende da entrada do algoritmo), e neste caso, a entrada é o tamanho de $\sigma$ mais os tamanhos da representação binária de $n, a_{0}, r, R, c$ e $\varepsilon$. Analogamente, definimos um algoritmo para o problema de separação fraca para a classe $\mathcal{K}$.

O seguinte Teorema de Grötschel, Lovász e Schrijver [GLS81] afirma que o problema de otimização fraca e o problema de separação fraca são equivalentes computacionalmente, isto é, se um problema pode ser solucionado em tempo polinomial, então o outro também pode ser.

Teorema 15 (Grötschel-Lovász-Schrijver, 1981). Seja $\mathcal{K}$ a classe dos corpos convexos. Existe um algoritmo em tempo polinomial que resolve o problema de otimização fraca para $\mathcal{K}$ se, e somente se, existe um algoritmo em tempo polinomial que resolve o problema de separação fraca para $\mathcal{K}$.

Dada uma matriz positiva definida $A$ e um vetor $a \in \mathbb{R}^{n}$, definimos o elipsóide

$$
E(A, a)=\left\{x \in \mathbb{R}^{n}:(x-a)^{t} A^{-1}(x-a) \leq 1\right\} .
$$

O volume de $E(A, a)$ é dado por $\operatorname{vol}(E(A, a))=\sqrt{\operatorname{det}(A)} V_{n}$, onde $V_{n}$ é o volume da bola unitária $B(0,1)$.

Há um outro resultado de Grötschel, Lovász e Schrijver [GLS81] que obtém uma outra versão do problema de otimização fraca a partir do problema de separação fraca para um corpo convexo.

Teorema 16. Seja $\sigma \in \Pi$ e $\mathcal{K}_{\sigma}$ um corpo convexo. Suponhamos que existe um algoritmo que resolve o problema de separação fraca para $\mathcal{K}_{\sigma}$. Então existe um algoritmo em tempo polinomial cuja entrada é um racional $\varepsilon>0$, e produz uma das seguintes afirmaçôes:

(a) um vetor a $\in \mathbb{R}^{n}$ tal que $\mathrm{d}\left(a, K_{\sigma}\right) \leq \varepsilon$;

(b) uma matriz positiva definida $A$ com entradas racionais e um vetor a $\in \mathbb{Q}^{n}$ tais que $K_{\sigma} \subseteq$ $E(A, a) e \operatorname{vol}(E(A, a)) \leq \varepsilon$.

Apresentamos agora um algoritmo em tempo polinomial que produz uma solução aproximada para programas semidefinidos. Definimos

$$
v_{\text {deep }}=\sup \{\langle C, X\rangle: B(X, \varepsilon) \subseteq \mathcal{F}\} .
$$

A partir do Teorema 16 e do Exemplo 14, obtemos o seguinte resultado para programas semidefinidos.

Teorema 17. Assumimos que o programa semidefinido (2.7) tem coeficientes racionais. Seja $R$ um racional positivo tal que $\|X\|_{F} \leq R$ para toda solução viável de (2.7), e seja $\varepsilon>0$ um número racional. Existe um algoritmo em tempo polinomial que produz uma das seguintes saídas.

(a) Uma matriz $X^{*} \in \mathcal{W}$ tal que $\left\|X^{*}-X\right\|_{F} \leq \varepsilon$ para alguma solução viável $X$, e $\left\langle C, X^{*}\right\rangle \geq$ $v_{\text {deep }}-\varepsilon$;

(b) Uma matriz positiva definida $A$ com entradas racionais e um vetor $a \in \mathbb{Q}^{n}$ tais que $\mathcal{F} \subseteq$ $E(A, a) \subseteq \mathcal{W}$ e $\operatorname{vol}(E(A, a)) \leq \varepsilon$.

\subsection{Grafos}

Um grafo é um par de conjuntos $(V, E)$ tal que $E \subseteq V^{(2)}=\{U \subseteq V:|U|=2\}$, isto é, os elementos de $E$ são subconjuntos de $V$ de cardinalidade dois. Os elementos de $V$ são chamados de vértices, e os elementos de $E$, arestas. A cardinalidade do conjunto de vértices de $G$ é dita ordem de $G$. Trabalharemos somente com grafos de ordem finita. 
Uma aresta $\{u, v\}$ é denotada por uv ou por $v u$. Dizemos que $u$ e $v$ são pontas de $u v$ e dizemos que $u$ e $v$ são vizinhos ou adjacentes.

Se $u \in X$ e $v \in Y$, onde $X$ e $Y$ são subconjuntos de $V$, então a aresta $u v$ é uma $X-Y$ aresta. $\mathrm{O}$ conjunto de todas as $X-Y$ arestas é um subconjunto de $E$ denotado por $E(X, Y)$. Denotemos por $e(X, Y)$ a cardinalidade de $E(X, Y)$.

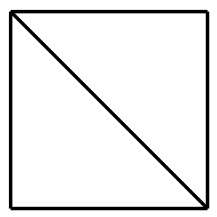

Figura 2.1: Exemplo de um grafo

Um caminho é um grafo não-vazio $P=(V, E)$ da forma

$$
V=\left\{x_{0}, x_{1} \ldots, x_{k}\right\}, E=\left\{x_{0} x_{1}, x_{1} x_{2}, \ldots, x_{k-1} x_{k}\right\},
$$

onde os $x_{i}$ são todos distintos. Um passeio em um grafo $G$ é um sequência alternada não-vazia $v_{0} e_{0} v_{1} e_{1} \ldots e_{k-1} v_{k}$ de vértices e arestas de $G$ tais que $e_{i}=v_{i} v_{i+1}$ para todo $i<k$. Note que todo passeio entre dois vértices contém um caminho entre esses vértices.

Um grafo orientado $D$ é um par de conjuntos disjuntos $(V, \vec{E})$ tal que $\vec{E} \subseteq V \times V$, e para todo $u, v \in V$, se $(u, v) \in E$, então $(v, u) \notin E$.

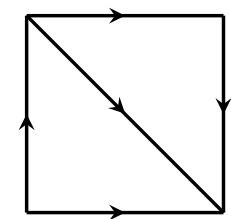

Figura 2.2: Exemplo de um grafo orientado

Seja $G=(V, E)$ um grafo. Para cada aresta $e \in E$ associaremos um valor real $w(e)$ chamado de peso. Então $G$, juntamente com cada peso das arestas, é chamado de grafo com pesos, e denotaremos por $G_{W}$. Aqui, o peso é um número não-negativo. A matriz de pesos $W$ associado ao grafo com pesos $G_{W}$ é uma matriz quadrada do tipo $V \times V$ tal que cada entrada $w_{i j}$ é igual a $w(e)$ se $e=i j$, e $w_{i j}=0$ caso contrário.

Para mais informações sobre a teoria dos grafos, recomendamos os livros [Bol79], [Bol98], [BM08] e [Die10].

\subsection{Complexidade Computacional}

A complexidade computacional é um ramo da matemática que estuda a eficiência dos algoritmos. Medimos a eficiência dos algoritmos estimando o tempo (número de operações) que o programa leva para encontrar uma resposta em função dos dados de entrada.

Os conceitos apresentados nesta seção serão vistos de maneira mais intuitiva, sem muito formalismo. Para mais informações sobre complexidade computacional, recomendamos os livros [Pap94], [AB09] e [KS95], onde se pode encontrar definições rigorosas.

Um problema de decisão é um problema cuja resposta é SIM ou NÃO.

Um algoritmo é dito determinístico se, dada uma determinada entrada (instância do problema), o algoritmo apresenta sempre a mesma saída e o mesmo tempo de execução. Um algoritmo é dito não-determinístico se, além dos comandos determinísticos usuais, o algoritmo pode usar o comando Escolha $(S)$ que retorna um elemento do conjunto $S$.

Um algoritmo que resolve um dado problema é dito polinomial se o seu tempo estimado no pior caso é limitado por uma função polinomial no tamanho da instância do problema. 
A classe $\mathrm{P}$ é formada por todos os problemas de decisão que podem ser resolvidos deterministicamente em tempo polinomial.

Exemplo 18 (Conectividade de um grafo). Dado um grafo $G$ e dois vértices $s, t \in G$, determinar se existe um caminho de s para t. Esse problema pertence a classe P. De fato, podemos construir um algoritmo que começa explorando as arestas do grafo a partir do vértice $s$, e explora todas as arestas não visitadas que são adjacentes às arestas previamente visitadas. Após no máximo $\left(\begin{array}{l}n \\ 2\end{array}\right)$ passos, ou uma das pontas das arestas exploradas é $t$, ou nenhuma delas tem ponta $t$.

A classe NP é formada por todos os problemas de decisão em que podemos verificar, em tempo polinomial, se um candidato de uma solução é uma solução.

Exemplo 19 (Soma do Subconjunto). Dado um número $N$ e um subconjunto dos naturais $X$ com $n$ elementos, decidir se existe um subconjunto de $X$ tal que a soma de seus elementos é igual a $N$.

Esse problema pertence à classe NP pois, se dermos um subconjunto particular de $X$, podemos facilmente verificar se a soma dos seus elementos é igual a $\mathrm{N}$.

Claramente $\mathrm{P} \subseteq \mathrm{NP}$, porém não se sabe se $\mathrm{P}=\mathrm{NP}$, e vários pesquisadores do assunto acreditam que essa relação seja falsa. O Instituto Clay de Matemática oferece um prêmio de um milhão de dólares pela solução da questão "P $=$ NP?". Note que se $\mathrm{P} \neq \mathrm{NP}$ então existe um algoritmo na classe NP e uma entrada para o qual esse algoritmo levará um tempo superpolinomial para terminar o processo.

Dizemos que um problema $\mathcal{P}$ é redutivel a um problema $\mathcal{Q}$ se existe um algoritmo de tempo polinomial que converte cada entrada $p \in \mathcal{P}$ a outra entrada $q \in \mathcal{Q}$ tal que $p \in \mathcal{P}$ se e somente se $q \in \mathcal{Q}$.

A classe NP-completo é definida da seguinte forma: um problema $\mathcal{P}$ pertence à classe NPcompleto se todos os problemas em NP são redutíveis para $\mathcal{P}$ em tempo polinomial.

Exemplo 20 (Caixeiro Viajante). Dado um conjunto finito $C=\left\{c_{1}, c_{2}, \ldots, c_{m}\right\}$ de "cidades", uma "distância" $\mathrm{d}\left(c_{i}, c_{j}\right) \in \mathbb{N}$ para cada par de cidades $c_{i}, c_{j} \in C$, e um inteiro positivo L, decidir se existe um passeio de Caixeiro Viajante, isto é, um passeio que visite todas as cidades e retorne à cidade de origem, de comprimento no máximo $L$.

Devemos ressaltar que a distância $\mathrm{d}\left(c_{i}, c_{j}\right)$ não é necessariamente igual a $\mathrm{d}\left(c_{j}, c_{i}\right)$, e não satisfaz a desigualdade triangular: $\mathrm{d}\left(c_{i}, c_{j}\right) \leq \mathrm{d}\left(c_{i}, c_{k}\right)+\mathrm{d}\left(c_{k}, c_{j}\right)$ para todo $i, j, k$.

O problema acima é NP-completo, porém está fora dos nossos estudos prová-lo. Podemos ver a prova em [Kar72].

Vale ressaltar aqui que o Problema da Soma do Subconjunto também é NP-completo, podemos ver a prova em [Kar72].

A classe NP-difícil é definida da seguinte forma: um problema $\mathcal{P}$ pertence à classe NP-difícil se todos os problemas em NP são redutíveis para $\mathcal{P}$ em tempo polinomial.

Note que um problema é NP-completo se pertence a NP e é NP-difícil.

Exemplo 21. Podemos reformular o Problema do Caixeiro Viajante da seguinte forma: dado um conjunto finito $C=\left\{c_{1}, c_{2}, \ldots, c_{m}\right\}$ de "cidades" e uma "distância" $\mathrm{d}\left(c_{i}, c_{j}\right) \in \mathbb{N}$ para cada par de cidades $c_{i}, c_{j} \in C$, encontrar um passeio de menor rota possivel que visite todas as cidades e retorne à cidade de origem. Esse problema é NP-difícil, e podemos encontrar a prova em [Kar72].

Fixe um conjunto finito $\Sigma$, chamado de alfabeto. O conjunto de todas as sequências finitas de elementos de $\Sigma$, chamadas de palavras, é denotado por $\Sigma^{*}$. Uma linguagem $L$ sobre o alfabeto $\Sigma$ é qualquer subconjunto $L \subseteq \Sigma^{*}$.

O teorema a seguir relaciona o problema "P $=$ NP"? com as classes dos problemas NP-difícil e NP-completa.

Teorema 22. Valem as seguintes asserções:

1. Se a linguagem $L$ é $N P$-difícil e $L \in P$, então $P=N P$; 
2. Se a linguagem $L$ é $N P$-completa, então $L \in P$ se e somente se $P=N P$.

Podemos ver a prova do Teorema 22 no livro [AB09].

O complemento de um problema de decisão é um problema de decisão trocando o papel da resposta SIM pela resposta NÃO.

A classe coNP é formada por todos os problemas de decisão para o qual seu complemento pertence a NP. Mais formalmente, a classe coNP é definida por

$$
\operatorname{coNP}=\left\{\Sigma^{*} \backslash L: L \subseteq \Sigma^{*} \text { e } L \in N P\right\} .
$$

Exemplo 23 (Complementar da Soma do Subconjunto). Dado um número $N$ e um subconjunto dos naturais $X$ com $n$ elementos, decidir se a soma dos elementos de cada um dos subconjuntos de $X$ não é igual a $N$.

Esse problema pertence à classe coNP pois é o complementar do Problema da Soma do Subconjunto, que pertence à classe NP.

Sabe-se que $\mathrm{P} \subseteq \mathrm{NP} \cap$ coNP, porém a igualdade ainda é um problema em aberto. Sabe-se também que se NP $\neq$ coNP então $\mathrm{P} \neq \mathrm{NP}$, e não se sabe ainda se a recíproca é verdadeira. Recomendamos o livro [Pap94] para ver a prova dessas relações.

A classe coNP-completa é definida da seguinte forma: um problema Q pertence à classe coNPcompleto se $Q$ pertence a coNP e todos os problemas em coNP são redutíveis para $\mathrm{Q}$ em tempo polinomial.

Existe uma relação entre a classe NP-completa e a classe coNP-completa.

Proposição 24. Se um problema é NP-completo, então seu complemento é coNP-completo.

Podemos ver a prova da Proposição 24 no livro [Pap94]

Além dos algoritmo determinísticos, existem os chamados algoritmos aleatorizados, que são algoritmos que fazem escolhas aleatórias durante sua execução. Um exemplo simples de um algoritmo aleatorizado é o quicksort aleatorizado.

Exemplo 25 (Quicksort aleatorizado). Considere o problema de ordenar um conjunto finito $C$ de números reais, o quicksort aleatorizado RandomQS $(C)$ executa os seguintes passos:

(1) Se C é vazio, retorne a lista vazia. Se C possui somente um elemento, retorne a lista com esse elemento. Suponhamos que $C$ tem mais de um elemento.

(2) Seja $x \in C$ escolhido uniformemente ao acaso. Compare todos os elementos restantes de $C$ com $x$, obtendo dois conjunto $C_{1}=\{y \in C: y<x\}$ e $C_{2}=\{y \in C: y>x\}$.

(3) Retorne a lista resultante de $\mathbf{R a n d o m Q S}\left(C_{1}\right)$ concatenada com a lista $\{y\}$ concatenada com a lista resultante de $\mathbf{R a n d o m} \mathbf{Q S}\left(C_{2}\right)$.

O quicksort aleatorizado é de fato um algoritmo aleatorizado pois temos uma escolha uniforme para tratar no passo (2).

Outros exemplos de algoritmos aleatorizados são o algoritmo de Goemans e Wiliamson [GW95] sobre corte máximo de um grafo, e o algoritmo de Alon e Naor [AN06] sobre o problema da norma de corte de uma matriz.

\subsection{Algoritmo de Aproximação}

Sabemos que a maioria do problemas importantes de otimização combinatória são NP-difíceis, e sabemos que, a menos que $P=N P$, não existe um algoritmo em tempo polinomial que encontra uma solução ótima para qualquer instância de um problema NP-difícil. Assim, para que haja um avanço nesses tipos de problemas, é interessante trabalhar com algoritmos em tempo polinomial que encontram soluções aproximadas desses problemas. 
Dado um real $\rho>0$ diferente de 1 , um algoritmo de $\rho$-aproximação para um problema de otimização é um algoritmo em tempo polinomial tal que para toda instância do problema, o algoritmo encontra uma solução cujo valor está limitado a um fator de $\rho$ do valor da solução ótima. Mais formalmente:

Definição 26. Sejam $\rho>0$ um real diferente de 1 e $X$ um problema de maximização (resp. minimização). Um algoritmo $\mathcal{A}$ é dito um algoritmo de $\rho$-aproximação para o problema $X$ se para toda instância $I$ de $X$ o algoritmo $\mathcal{A}$ produz uma solução viável de uma função objetivo $\mathcal{A}(I)$ em tempo polinomial tal que

$$
\begin{aligned}
& \operatorname{OPT}(I) \leq \mathcal{A}(I) \leq \rho \operatorname{OPT}(I), \text { se } \rho>1 \\
& \rho \operatorname{OPT}(I) \leq \mathcal{A}(I) \leq \operatorname{OPT}(I), \text { se } \rho<1 .
\end{aligned}
$$

Dizemos que $\rho$ é a garantia de desempenho do algoritmo de $\rho$-aproximação.

Para problemas de maximização, convencionamos restringir $\rho<1$; e para problemas de minimização, $\rho>1$.

Note que, para problemas de maximização, quanto mais a garantia de desempenho for próxima de 1, teremos uma aproximação mais próxima do valor da solução ótima. Mas em geral, como estamos trabalhando com todas as instâncias, pode ser que exista um conjunto $I^{\prime}$ de instâncias que faça a garantia de desempenho $\rho$ ser menor que um certo $\rho_{0}<1$, enquanto que, se trabalhássemos com as instâncias $I \backslash I^{\prime}$, a garantia de desempenho seria $\rho>\rho_{0}$. Mas mesmo assim, existem aqueles algoritmos que não tem esse problema, como definimos a seguir.

Definição 27. Um esquema de aproximação em tempo polinomial (Polynomial-time approximation scheme - PTAS) é uma família de algoritmos $\left\{A_{\varepsilon}\right\}$, na qual existe um algoritmo $A_{\varepsilon}$ para cada $0<$ $\varepsilon<1$, tais que $A_{\varepsilon}$ é um algoritmo de $(1+\varepsilon)$-aproximação para problemas de minimização, ou um algoritmo de $(1-\varepsilon)$-aproximação para problemas de maximização.

Existem vários problemas que possuem PTAS, como por exemplo, o Problema da Mochila.

Exemplo 28 (Problema da Mochila). Sejam $X=\left\{x_{1}, \ldots, x_{n}\right\}$ um conjunto de $n$ itens tais que cada uma delas tem um peso positivo peso $\left(x_{i}\right)$ e um lucro positivo lucro $\left(x_{i}\right)$. Ademais, possuímos uma mochila que pode carregar os itens até totalizar peso $W$. Para um subconjunto $S \subseteq X$, definimos $\operatorname{peso}(S)=\sum_{x \in S} \operatorname{peso}(x)$ e lucro $(S)=\sum_{x \in S}$ lucro $(x)$. O problema consiste em carregar na mochila uma quantidade de itens que maximiza o lucro de forma que o peso não passe de $W$.

A prova que o problema da mochila é PTAS pode ser vista em [Sah75].

Por outro lado, existe a classe dos problemas MAX SNP-difícil, introduzida por Papadimitriou e Yannakakis [PY91].

Definição 29. Dado um inteiro positivo $r$, definimos o problema MAX-r-FUNCTION-SAT da seguinte forma: a entrada consiste de $m$ funções Booleanas $f_{1}, f_{2}, \ldots, f_{m}$ em $n$ variáveis, e cada $f_{i}$ depende somente de $r$ variáveis, para algum $r$ fixo. A saída é uma atribuição de valores verdade para as $n$ variáveis, de modo que satisfaça o maior número de $f_{i}$ possivel.

O problema MAX SNP é um problema MAX-r-FUNCTION-SAT para algum $r$ fixo.

Temos o seguinte teorema de MAX SNP-difícil, provado em [PY91].

Teorema 30. Para todo problema MAX SNP-difícil, não existe um esquema de aproximação em tempo polinomial, a menos que $P=N P$.

Um exemplo de um problema MAX SNP-difícil é o Problema do Corte Máximo: Dado um grafo, encontrar um subconjunto $S$ dos vértices tal que maximiza o número de arestas que tem uma ponta em $S$ e outra em $V \backslash S$. Veja Seção 2.8 para mais detalhes. No Capítulo 5 mostraremos que o problema de calcular a norma de corte para matrizes arbitrárias é MAX SNP-difícil.

Graham foi o primeiro que trabalhou com algoritmos de aproximação em tempo polinomial [Gra66], que trata de um sistema de multiprocessamento composto de várias unidades de processadores idênticos operando em paralelo. Porém o conceito formal de algoritmo de aproximação foi dado 
por Garey, Graham e Ullman [GGU72]. O termo "esquema de aproximação em tempo polinomial" foi devido a Garey e Johnson [GJ78].

Para mais informações sobre algoritmos de aproximação, recomendamos os livros [Vaz01] e [Gon07].

\subsection{Corte de grafo}

Dado um grafo $G=(V, E)$ e $X \subseteq V$, o corte de $X$ é a cardinalidade do conjunto das $X-X^{c}$ arestas. Definimos o corte máximo e corte mínimo de $G$, respectivamente, por

$$
\begin{aligned}
\operatorname{MAXCUT}(G) & =\max \left\{e\left(X, X^{c}\right): X \subseteq V\right\}, \\
\operatorname{MinCUT}(G) & =\min \left\{e\left(X, X^{c}\right): X \subseteq V\right\} .
\end{aligned}
$$

O corte máximo e mínimo de um grafo com peso $G_{W}=(V, E)$ é definido da seguinte forma: Particionando o conjunto $V$ em duas partes $V^{-}$e $V^{+}$, o peso de corte de $\left(V^{-}, V^{+}\right)$é definido como

$$
w\left(V^{-}, V^{+}\right)=\sum_{(u, v) \in V^{-} \times V^{+}} w_{u v}
$$

O corte máximo de $G_{W}$ é o maior valor de $w\left(V^{-}, V^{+}\right)$sobre toda partição $\left(V^{-}, V^{+}\right)$de $V$, e denotamos por $\operatorname{MAXCUT}(W)$. Analogamente, definimos $\operatorname{MINCUT}(W)$, o corte mínimo de $D$, como o menor valor de $w\left(V^{-}, V^{+}\right)$sobre toda partição $\left(V^{-}, V^{+}\right)$de $V$.

Note que tomando $w_{u v}=1$ se $u v \in E$, então $\operatorname{MAXCUT}(W)=\operatorname{MAXCUT}(G) \operatorname{e} \operatorname{MinCUT}(W)=$ MINCUT $(G)$, onde $G$ é o grafo obtido do grafo orientado $D$ removendo as orientações.

Podemos escrever o peso de corte de uma partição $\left(V^{-}, V^{+}\right)$de $V$ de uma outra forma. Para todo vértice $u \in V$, associamos $x_{u} \in\{-1,1\}$ satisfazendo

$$
x_{u}=\left\{\begin{array}{l}
-1, \text { se } u \in V^{-} \\
1, \text { se } u \in V^{+}
\end{array}\right.
$$

Claramente, para quaisquer dois vértices $u, v \in V$ temos $1-x_{u} x_{v} \in\{0,2\}$, e é igual a zero se os vértices $u$ e $v$ pertencem à mesma parte, e é igual a dois se $u$ e $v$ pertencem às partes distintas. Dessa forma, podemos expressar o peso de corte como

$$
w\left(V^{-}, V^{+}\right)=\frac{1}{2}\left(\frac{1}{2} \sum_{u, v \in V} w_{u v}\left(1-x_{u} x_{v}\right)\right) .
$$

Logo, podemos formular o Problema do Corte Máximo com Peso e o Problema do Corte Mínimo com Peso como calcular o valor exato das funções dadas por

$$
\begin{aligned}
& \operatorname{MAXCUT}(W)=\max \left\{\frac{1}{4} \sum_{u, v \in V} w_{u v}\left(1-x_{u} x_{v}\right): x_{u}^{2}=1, u \in V\right\} \\
& \operatorname{MinCUT}(W)=\min \left\{\frac{1}{4} \sum_{u, v \in V} w_{u v}\left(1-x_{u} x_{v}\right): x_{u}^{2}=1, u \in V\right\} .
\end{aligned}
$$

O Problema do Corte Mínimo com peso de um grafo é um problema que pode ser resolvido em tempo polinomial. Isso se deve ao Teorema do Fluxo Máximo-Corte Mínimo, o qual afirma que em um fluxo de rede, o valor máximo do fluxo passando de uma origem até um destino é igual à sua capacidade mínima de um corte da rede. Podemos ver com mais detalhes no Capítulo 3 e no Capítulo 6 de Diestel [Die10].

Por outro lado, o Problema do Corte Máximo com Peso é um dos problemas NP-completo de 
Karp [Kar72]. Sabe-se também que o caso sem peso é também NP-completo [GJS76]. O problema do corte máximo é solúvel em tempo polinomial em algumas classes de grafos, como por exemplo para grafos planares [OD72] [Had75].

Como não se sabe se existe um algoritmo eficiente que calcule o corte máximo de um grafo (e caso existir vai implicar que $\mathrm{P}=\mathrm{NP}$ ), um outro meio de trabalhar com o problema é encontrar um algoritmo de $\rho$-aproximação.

Há vários resultados de algoritmo de $\rho$-aproximação para o Problema do Corte Máximo ao longo do tempo.

\begin{tabular}{|c|c|}
\hline$\frac{1}{2}$ & Gonzales e Shani, 1976 [SG76] \\
\hline$\frac{1}{2}+\frac{1}{2 m}$ & Vitányi, 1981 [Vit81] \\
\hline$\frac{1}{2}+\frac{n-1}{4 m}$ & Poljak e Turzík, 1982 [PT82] \\
\hline$\frac{1}{2}+\frac{1}{2 n}$ & Haglin e Venkatesan, 1991 [HV91] \\
\hline$\frac{1}{2}+\frac{1}{2 \Delta}$ & Hofmeister e Lefmann, 1996 [HL96] \\
\hline
\end{tabular}

onde $n=|V|, m=|E|$ e $\Delta$ é o grau máximo.

Em 1995, Goemans e Williamson apresentaram em [GW95] um algoritmo de $(\alpha-\varepsilon)$-aproximação para o Problema o Corte Máximo, onde

$$
\alpha=\min _{0 \leq \theta \leq \pi} \frac{2}{\pi} \cdot \frac{\theta}{1-\cos \theta} \cong 0.87856
$$

e $\varepsilon$ é um escalar positivo qualquer. Essa garantia de desempenho é a melhor até então.

Håstad [Hås01] provou que, se $P \neq N P$, então não existe um algoritmo de aproximação em tempo polinomial para o Problema do Corte Máximo com garantia de desempenho excedendo $16 / 17 \cong 0.941$. 


\section{Capítulo 3}

\section{Norma de Corte}

A norma de corte foi introduzida por Frieze e Kannan em [FK99] como ferramenta para obter um método de decomposição matricial (veja Seção 5.3 para mais detalhes). A Decomposição Matricial de Frieze e Kannan possui várias aplicações, entre elas, uma versão construtiva do Lema da Regularidade de Szemerédi, aproximação do corte máximo de um grafo, Problema da Condutância de um Grafo e Problema do Subgrafo Máximo Acíclico.

Na Seção 3.1 estudaremos as propriedades da norma de corte. Mostraremos que ela é equivalente à norma que é valor ótimo de um certo programa inteiro quadrático. Apresentaremos também um programa semidefinido que será útil ao longo deste trabalho. Na Seção 3.2 apresentaremos um algoritmo de aproximação da norma de corte que se baseia na prova original do Teorema de Grothendieck. Na Seção 3.3 exibiremos um outro algoritmo de aproximação para a norma de corte, porém com garantia de desempenho melhor que a da Seção 3.2. Esse algoritmo se baseia na prova de Rietz do Teorema de Grothendieck.

\subsection{A norma de corte e suas propriedades}

Definição 31. Seja $A=\left(a_{i j}\right)$ uma matriz do tipo $m \times n$. A norma de corte de $A$ é definida por

$$
\|A\|_{c u t}=\max _{S \subset[m], T \subset[n]}\left|\sum_{\substack{i \in S \\ j \in T}} a_{i j}\right| .
$$

Daqui para a frente do nosso trabalho, vamos assumir que $A$ tem entradas racionais. Assumimos esta hipótese porque os resultados que vamos estudar são computacionais.

Definiremos a seguinte norma que será importante para o nosso estudo daqui para frente. Dada uma matriz real $A=\left(a_{i j}\right)$ do tipo $m \times n$, definimos a norma

$$
\|A\|_{\infty \rightarrow 1}=\max \left\{\sum_{i j} a_{i j} \varepsilon_{i} \delta_{j}:\left\{\varepsilon_{i}\right\}_{i=1}^{m},\left\{\delta_{j}\right\}_{j=1}^{n} \subseteq\{-1,1\}\right\},
$$

onde $\sum_{i j}$ é igual a $\sum_{i=1}^{m} \sum_{j=1}^{n}$, e $\{\cdot\}_{i=1}^{m}$ e $\{\cdot\}_{j=1}^{n}$ são multiconjuntos, isto é, pode ter repetição de elementos. Observe que o máximo é não-negativo, pois, para todo $\varepsilon_{i}, \delta_{j}$, podemos tomar $\tilde{\varepsilon}_{i}=-\varepsilon_{i}$ para todo $i$. Assim

$$
\sum_{i j} a_{i j} \tilde{\varepsilon}_{i} \delta_{j}=-\sum_{i j} a_{i j} \varepsilon_{i} \delta_{j}
$$

Note que a norma $\|A\|_{\infty \rightarrow 1}$ é o valor ótimo do seguinte programa inteiro quadrático (Sugerimos o 
livro [Sch03] para mais informações sobre programação inteira quadrática):

$$
\begin{aligned}
& \max \sum_{i j} a_{i j} x_{i} y_{j} \\
& \text { s.a } x_{i}, y_{j} \in\{-1,1\} \text { para todo } i, j .
\end{aligned}
$$

O lema a seguir será útil para descrever uma caracterização da norma $\|\cdot\|_{\infty \rightarrow 1}$.

Lema 32. Seja $n$ um inteiro positivo. Considere a bola $B_{l_{\infty}^{n}}=\left\{x \in \mathbb{R}^{n}:\|x\|_{\infty} \leq 1\right\}$ e, para todo $S \subseteq[n]$, defina $\chi^{S}=\left(\chi_{i}^{S}\right)_{i=1}^{n}$ o vetor em $\mathbb{R}^{n}$ dado por

$$
\chi_{i}^{S}=\left\{\begin{array}{l}
1, \text { se } i \in S \\
-1, \text { caso contrário. }
\end{array}\right.
$$

Considere o conjunto $C=\operatorname{conv}\left\{\chi^{S}: S \subseteq[n]\right\}$ o fecho convexo do conjunto dos vetores $\chi^{S}$ sobre todos os $S \subseteq[n]$. Temos $C=B_{l_{\infty}^{n}}$.

Demonstração. Como $B_{l_{\infty}^{n}}$ é convexo e contém os vetores $\chi^{S}$ para todo $S \subseteq[n]$, temos $C \subseteq B_{l_{\infty}^{n}}$.

Seja $x \in B_{l_{\infty}^{n}}$. Como o conjunto $B_{l_{\infty}^{n}}$ é convexo, existem $\left(\alpha_{S}\right)_{S \subseteq[n]} \in \mathbb{R}$ não-negativos tais que $\sum_{S \subseteq[n]} \alpha_{S}=1$ e $\sum_{S \subseteq[n]} \alpha_{S} \chi^{S}=x$. Como $C$ contém os vetores $\chi^{S}$ para todo $S \subseteq[n]$ e é convexo, concluímos que $\sum_{S \subseteq[n]} \alpha_{S} \chi^{S} \in C$. Portanto $C=B_{l_{\infty}^{n}}$.

Vamos ver agora que a norma $\|A\|_{\infty \rightarrow 1}$ pode ser vista como a norma de $A$ onde $A$ é um operador linear de $l_{\infty}^{m}$ para $l_{1}^{n}$, onde $l_{\infty}^{m}$ é o espaço dos vetores em $\mathbb{R}^{m}$ munido da norma $\|\cdot\|_{\infty}$, e $l_{1}^{n}$ é o espaço dos vetores em $\mathbb{R}^{n}$ munido da norma $\|\cdot\|_{1}$. Lembremos que, se $x=\left(x_{1}, \ldots, x_{n}\right)$, então

$$
\|x\|_{1}=\sum_{i=1}^{n}\left|x_{i}\right|
$$

Proposição 33. Seja A uma matriz do tipo $m \times n$. Temos

$$
\max _{x \in B_{l_{\infty}^{n}}}\|A x\|_{1}=\|A\|_{\infty \rightarrow 1}
$$

Demonstração. Como o máximo de $\|A x\|_{1}$ sobre o conjunto $C$ descrito no Lema 32 é atingido em algum vetor $\tilde{x}=\left(\tilde{x}_{j}\right)_{j=1}^{n}=\left(\chi_{j}^{S}\right)_{j=1}^{n}$, com $S \subseteq[n]$, basta mostrar que $\|A \tilde{x}\|_{1}=\|A\|_{\infty \rightarrow 1}$.

Para cada $i \in[m]$, existe $\varepsilon_{i} \in\{-1,1\}$ tal que

$$
\left|\sum_{j=1}^{n} a_{i j} \tilde{x}_{j}\right|=\sum_{j=1}^{n} a_{i j} \varepsilon_{i} \tilde{x}_{j} .
$$

Portanto,

$$
\|A \tilde{x}\|_{1}=\sum_{i=1}^{m}\left|\sum_{j=1}^{n} a_{i j} \tilde{x}_{j}\right|=\sum_{i j} a_{i j} \varepsilon_{i} \tilde{x}_{j} \leq\|A\|_{\infty \rightarrow 1} .
$$

Sejam $\left\{\varepsilon_{i}\right\}_{i=1}^{m},\left\{\delta_{j}\right\}_{j=1}^{n} \subseteq\{-1,1\}$ tais que

$$
\|A\|_{\infty \rightarrow 1}=\sum_{i j} a_{i j} \varepsilon_{i} \delta_{j}
$$

Assim,

$$
\sum_{i j} a_{i j} \varepsilon_{i} \delta_{j}=\sum_{i=1}^{m} \varepsilon_{i} \sum_{j=1}^{n} a_{i j} \delta_{j} \leq \sum_{i=1}^{m}\left|\sum_{j=1}^{n} a_{i j} \delta_{j}\right| \leq\|A \tilde{x}\|_{1}
$$


como queríamos

Mostremos que a norma de corte e a norma $\|\cdot\|_{\infty \rightarrow 1}$ são equivalentes. Uma consequência do lema a seguir é que o problema de aproximar $\|A\|_{\text {cut }}$ em tempo polinomial é equivalente ao problema de aproximar $\|A\|_{\infty \rightarrow 1}$ em tempo polinomial.

Lema 34. Seja $A=\left(a_{i j}\right)$ uma matriz do tipo $m \times n$. Temos

$$
\|A\|_{c u t} \leq\|A\|_{\infty \rightarrow 1} \leq 4\|A\|_{c u t} .
$$

Demonstração. Para cada conjunto $\left\{\varepsilon_{i}\right\}_{i=1}^{m} \subseteq\{-1,1\}$ e $\left\{\delta_{j}\right\}_{j=1}^{n} \subseteq\{-1,1\}$, definimos os conjuntos $S^{+}, S^{-} \subseteq[m]$ e $T^{+}, T^{-} \subseteq[n]$ como $S^{ \pm}=\left\{i \in[m]: \varepsilon_{i}= \pm 1\right\}$ e $T^{ \pm}=\left\{j \in[n]: \delta_{j}= \pm 1\right\}$. Pela desigualdade triangular,

$$
\left|\sum_{i j} a_{i j} \varepsilon_{i} \delta_{j}\right|=\left|\sum_{\substack{i \in S^{+} \\ j \in T^{+}}} a_{i j}+\sum_{\substack{i \in S^{-} \\ j \in T^{-}}} a_{i j}-\sum_{\substack{i \in S^{+} \\ j \in T^{-}}} a_{i j}-\sum_{\substack{i \in S^{-} \\ j \in T^{+}}} a_{i j}\right| \leq 4\|A\|_{c u t} .
$$

Tomando $\left\{\varepsilon_{i}\right\}_{i=1}^{m}$ e $\left\{\delta_{j}\right\}_{j=1}^{n}$ que maximiza $\sum_{i j} a_{i j} \varepsilon_{i} \delta_{j}$, obtemos a desigualdade da direita.

Suponhamos agora que $\|A\|_{c u t}=\left|\sum_{i \in S_{0}, j \in T_{0}} a_{i j}\right|$. Definimos $x_{i}=1$ se $i \in S_{0}$ e $x_{i}=-1$ caso contrário, e analogamente, $y_{j}=1$ se $j \in T_{0}$ e $y_{j}=-1$ caso contrário. Temos

$$
\left|\sum_{\substack{i \in S_{0} \\ j \in T_{0}}} a_{i j}\right|=\left|\sum_{i j} a_{i j} \frac{x_{i}+1}{2} \frac{y_{j}+1}{2}\right|=\left|\frac{1}{4} \sum_{i j} a_{i j}+\frac{1}{4} \sum_{i j} a_{i j} x_{i}+\frac{1}{4} \sum_{i j} a_{i j} y_{j}+\frac{1}{4} \sum_{i j} a_{i j} x_{i} y_{j}\right| .
$$

O valor absoluto de cada um dos quatro termos do lado direito da igualdade é no máximo $\|A\|_{\infty \rightarrow 1} / 4$. Portanto $\|A\|_{\text {cut }} \leq\|A\|_{\infty \rightarrow 1}$.

Como a norma de corte e a norma $\|\cdot\|_{\infty \rightarrow 1}$ são equivalentes, obtemos o seguinte resultado: Dada uma matriz $A=\left(a_{i j}\right)$ do tipo $m \times n$, se existe um algoritmo polinomial que encontra $\varepsilon_{i}, \delta_{j} \in\{-1,1\}$ tais que

$$
\sum_{i j} a_{i j} \varepsilon_{i} \delta_{j} \geq \rho\|A\|_{\infty \rightarrow 1}
$$

tomando os conjuntos $S^{+}, S^{-} \subseteq[m]$ e $T^{+}, T^{-} \subseteq[n]$ como $S^{ \pm}=\left\{i \in[m]: \varepsilon_{i}= \pm 1\right\}$ e $T^{ \pm}=\{j \in$ $\left.[n]: \delta_{j}= \pm 1\right\}$, obtemos

$$
\sum_{i j} a_{i j} \varepsilon_{i} \delta_{j} \leq 4 \max \left\{\left|\sum_{\substack{i \in S \\ j \in T}} a_{i j}\right|: S \in\left\{S^{+}, S^{-}\right\}, T \in\left\{T^{+}, T^{-}\right\}\right\} .
$$

Logo, sendo $S_{0}$ e $T_{0}$ os conjuntos que maximizam o lado direito da desigualdade acima, e sabendo que $\|A\|_{\infty \rightarrow 1} \geq\|A\|_{\text {cut }}$ temos

$$
\left|\sum_{\substack{i \in S_{0} \\ j \in T_{0}}} a_{i j}\right| \geq \frac{\rho}{4}\|A\|_{c u t}
$$

Portanto, existe um algoritmo de aproximação com garantia de desempenho $\rho / 4$ para a norma de corte.

Seja $H$ um espaço de Hilbert. Denotemos por $B_{H}$ o conjunto dos vetores em $H$ de norma unitária, onde a norma é vinda do produto interno. Dada uma matriz $A=\left(a_{i j}\right)$ do tipo $m \times n$, 
definimos

$$
\operatorname{SDP}(A)=\max \left\{\sum_{i j} a_{i j}\left\langle u_{i}, v_{j}\right\rangle:\left\{u_{i}\right\}_{i=1}^{m},\left\{v_{j}\right\}_{j=1}^{n} \subseteq B_{H}\right\} .
$$

Como $m+n$ vetores em $H$ geram um subespaço vetorial de dimensão no máximo $m+n$ o qual é isométrico ao espaço vetorial $\mathbb{R}^{m+n}$ munido da norma usual, podemos assumir sem perda de generalidade que o conjunto de vetores $\left\{u_{i}\right\}_{i=1}^{m}\left\{v_{j}\right\}_{j=1}^{n}$ está contido na esfera de raio unitário $S^{m+n-1}$.

É claro que $\operatorname{SDP}(A) \geq\|A\|_{\infty \rightarrow 1}$, pois a função $f:\{-1,1\} \rightarrow S^{m+n-1}$ dada por $f(\varepsilon)=$ $(\varepsilon, 0, \ldots, 0)$ mostra que o conjunto das soluções viáveis de (3.4) "contém" o conjunto das soluções viáveis de (3.2). Assim

$$
\sum_{i j} a_{i j} \varepsilon_{i} \delta_{j}=\sum_{i j} a_{i j}\left\langle f\left(\varepsilon_{i}\right), f\left(\delta_{j}\right)\right\rangle .
$$

Note também que o valor $\operatorname{SDP}(A)$ é valor ótimo do programa:

$$
\begin{aligned}
& \max \sum_{i j} a_{i j}\left\langle u_{i}, v_{j}\right\rangle \\
& \text { s.a. }\left\|u_{i}\right\|_{2}=\left\|v_{j}\right\|_{2}=1 \text { para todo } i, j .
\end{aligned}
$$

Note que (3.5) é uma relaxação do programa inteiro quadrático (3.3).

Vamos mostrar que o programa (3.5) pode ser expresso como um de programa semidefinido.

Proposição 35. Para toda matriz $A$ do tipo $m \times n$, temos que $\operatorname{SDP}(A)$ é valor ótimo do programa semidefinido abaixo:

$$
\begin{aligned}
\max & \frac{1}{2}\left\langle\left(\begin{array}{cc}
0 & A \\
A^{t} & 0
\end{array}\right), X\right\rangle \\
\text { s.a. } & \operatorname{diag}(X)=1 ; \\
& X \succeq 0 ; \\
& X \in \mathbb{R}^{(m+n) \times(m+n)},
\end{aligned}
$$

onde $\mathbf{1}=(1, \ldots, 1) \in \mathbb{R}^{m+n}$ e $\operatorname{diag}(X)$ é a diagonal principal de $X$.

Demonstração. Sejam $u_{1}, \ldots, u_{m}, v_{1}, \ldots, v_{n} \in S^{m+n-1}$ vetores unitários tais que

$$
\operatorname{SDP}(A)=\sum_{i j} a_{i j}\left\langle u_{i}, v_{j}\right\rangle
$$

Denotemos por $v_{j}=u_{m+j}$ para cada $j \in[n]$ e seja $x_{i, j}=\left\langle u_{i}, u_{j}\right\rangle$ com $i, j \in[m+n]$. Temos que a matriz $X=\left(x_{i, j}\right)_{1 \leq i, j \leq m+n}$ é positiva semidefinida pelo item $(i i)$ da Proposição 2 . Como $x_{i, i}=$ $\left\|u_{i}\right\|_{2}^{2}=1$, temos $\operatorname{diag}(X)=\mathbf{1}$. 
Seja $\tilde{A}=2^{-1}\left(\begin{array}{cc}0 & A \\ A^{t} & 0\end{array}\right)$, temos

$$
\begin{aligned}
\langle\tilde{A}, X\rangle & =\frac{1}{2}\left(\sum_{i j} a_{i j} x_{i, m+j}+\sum_{i j} a_{i j} x_{m+j, i}\right) \\
& =\sum_{i j} a_{i j}\left(\frac{x_{i, m+j}+x_{m+j, i}}{2}\right) \\
& =\sum_{i j} a_{i j}\left(\frac{\left\langle u_{i}, u_{m+j}\right\rangle+\left\langle u_{m+j}, u_{i}\right\rangle}{2}\right) \\
& =\sum_{i j} a_{i j}\left\langle u_{i}, u_{m+j}\right\rangle \\
& =\sum_{i j} a_{i j}\left\langle u_{i}, v_{j}\right\rangle \\
& =\operatorname{SDP}(A) .
\end{aligned}
$$

Portanto, $v_{\text {primal }} \geq \operatorname{SDP}(A)$.

Reciprocamente, seja $X=\left(x_{i, j}\right)$ uma solução ótima para o problema (3.6). Temos que $X$ é uma matriz positiva semidefinida. Logo, pelo item $(i i)$ da Proposição 2 , existem vetores $u_{1}, \ldots, u_{m+n} \in$ $\mathbb{R}^{m+n}$ tais que $x_{i, j}=\left\langle u_{i}, u_{j}\right\rangle$. Ademais, como $\operatorname{diag}(X)=\mathbf{1}$, temos $1=x_{i, i}=\left\|u_{i}\right\|^{2}$. Tomando $v_{j}=$ $u_{m+j}$ com $1 \leq j \leq n$, os vetores $u_{1}, \ldots, u_{m}, v_{1}, \ldots, v_{n}$ satisfazem

$$
\sum_{i j} a_{i j}\left\langle u_{i}, v_{j}\right\rangle=\frac{1}{2}\left\langle\left(\begin{array}{cc}
0 & A \\
A^{t} & 0
\end{array}\right), X\right\rangle=v_{\text {primal }} .
$$

Portanto, $v_{\text {primal }} \leq \mathrm{SDP}(A)$.

\subsection{Aproximação determinística da norma de corte}

Apresentaremos aqui um algoritmo de $\rho$-aproximação determinístico em tempo polinomial para o Problema da Norma de Corte $\operatorname{com} \rho=0.03$. A prova do teorema se baseia na prova da Desigualdade de Grothendieck em [Gro53]. Apesar da garantia de desempenho ser pequena, os métodos que foram usados na prova são interessantes.

Teorema 36 (Alon-Naor, 2006). Existe um algoritmo em tempo polinomial que produz, para toda matriz $A=\left(a_{i j}\right)$ do tipo $m \times n$, inteiros $\left\{\varepsilon_{i}\right\}_{i=1}^{m},\left\{\delta_{j}\right\}_{j=1}^{n} \subseteq\{-1,1\}$ tais que

$$
\sum_{i j} a_{i j} \varepsilon_{i} \delta_{j} \geq 0.03 \operatorname{SDP}(A)
$$

Sabendo que $\operatorname{SDP}(A) \geq\|A\|_{\infty \rightarrow 1}$, o Teorema 36 garante que existe um algoritmo em tempo polinomial que produz inteiros $\left\{\varepsilon_{i}\right\}_{i=1}^{m},\left\{\delta_{j}\right\}_{j=1}^{n} \subseteq\{-1,1\}$ tais que

$$
\sum_{i j} a_{i j} \varepsilon_{i} \delta_{j} \geq 0.03\|A\|_{\infty \rightarrow 1}
$$

Para demonstrar o Teorema 36, necessitaremos do seguinte: Sejam $p=m+n$ e $V$ um conjunto de $t=O\left(p^{2}\right)$ vetores $\varepsilon=\left(\varepsilon_{1}, \ldots, \varepsilon_{p}\right) \in\{-1,1\}^{p}$ tal que para cada quatro coordenadas distintas $1 \leq$ 
$i_{1}<i_{2}<i_{3}<i_{4} \leq p$ e cada escolha de $\delta_{1}, \ldots, \delta_{4} \in\{-1,1\}$, exatamente $t / 16$ vetores em $V$ tem $\varepsilon_{i_{k}}=\delta_{k}$, com $1 \leq k \leq 4$. O conjunto $V$ é chamado de Orthogonal Array of Strength 4, e o método para construir esse conjunto pode ser visto em [ABI86] e [AS08].

Considere $V$ como um espaço amostral para o qual os elementos estão distribuídos uniformemente. Para cada vetor unitário $q=\left(q_{1}, \ldots, q_{p}\right) \in \mathbb{R}^{p}$, definimos $H(q)$ a variável aleatória definida sobre o espaço $V$ dada por

$$
[H(q)](\varepsilon)=\sum_{j=1}^{p} \varepsilon_{j} q_{j} .
$$

Proposição 37. Dados $q, q^{\prime} \in \mathbb{R}^{p}$ dois vetores unitários, o valor esperado de $H(q) H\left(q^{\prime}\right)$ é igual ao produto interno $\left\langle q, q^{\prime}\right\rangle$.

Demonstração. Sejam $q=\left(q_{1}, \ldots, q_{p}\right)$ e $q^{\prime}=\left(q_{1}^{\prime}, \ldots, q_{p}^{\prime}\right)$ dois vetores unitários. Como $V$ é um espaço amostral tal que os elementos são distribuídos uniformemente, segue que

$$
\begin{aligned}
\mathbb{E}\left[H(q) H\left(q^{\prime}\right)\right] & =\frac{1}{t} \sum_{\varepsilon \in V} H(q)(\varepsilon) H\left(q^{\prime}\right)(\varepsilon) \\
& =\frac{1}{t} \sum_{i, j=1}^{p} q_{i} q_{j}^{\prime}\left(\sum_{\varepsilon \in V} \varepsilon_{i} \varepsilon_{j}\right) \\
& =\frac{1}{t} \sum_{i=1}^{p} q_{i} q_{i}^{\prime}\left(\sum_{\varepsilon \in V} \varepsilon_{i}^{2}\right)+\frac{1}{t} \sum_{\substack{i, j=1 \\
i \neq j}}^{p} q_{i} q_{j}^{\prime}\left(\sum_{\varepsilon \in V} \varepsilon_{i} \varepsilon_{j}\right)
\end{aligned}
$$

Note que $\sum_{\varepsilon \in V} \varepsilon_{i}^{2}=t$. Pela definição do conjunto $V$, para quaisquer duas coordenadas distintas $i_{1}, i_{2}$ e cada $\delta_{1}, \delta_{2} \in\{-1,1\}$, exatamente $t / 4$ vetores em $V$ tem $\varepsilon_{i_{k}}=\delta_{k}$ com $k=1,2$. Assim,

$$
\sum_{\varepsilon \in V} \varepsilon_{i} \varepsilon_{j}=2 \frac{t}{4}+(-1) 2 \frac{t}{4}=0
$$

Portanto $\mathbb{E}\left[H(q) H\left(q^{\prime}\right)\right]=\left\langle q, q^{\prime}\right\rangle$, como queríamos.

Em particular, o valor esperado de $[H(q)]^{2}$ é igual a 1. Agora vamos calcular uma cota superior para o valor esperado de $[H(q)]^{4}$.

Proposição 38. Dado $q \in \mathbb{R}^{p}$ um vetor unitário, o valor esperado de $[H(q)]^{4}$ é no máximo 3 .

Demonstração. Seja $q=\left(q_{1}, \ldots, q_{p}\right) \in \mathbb{R}^{p}$ um vetor unitário. Como $V$ é um espaço amostral tal que os elementos são distribuídos uniformemente, segue que o valor esperado de $[H(q)]^{4}$ é igual a

$$
\begin{aligned}
\mathbb{E}\left([H(q)]^{4}\right) & =\frac{1}{t} \sum_{\varepsilon \in V}[H(q)(\varepsilon)]^{4} \\
& =\frac{1}{t} \sum_{i, j, k, l=1}^{p} q_{i} q_{j} q_{k} q_{l}\left(\sum_{\varepsilon \in V} \varepsilon_{i} \varepsilon_{j} \varepsilon_{k} \varepsilon_{l}\right) \\
& =\frac{1}{t} \sum_{i=1}^{p} q_{i}^{4}\left(\sum_{\varepsilon \in V} \varepsilon_{i}^{4}\right)+\frac{1}{t}\left(\begin{array}{l}
4 \\
2
\end{array}\right) \sum_{i, j=1}^{p} q_{i}^{2} q_{j}^{2}\left(\sum_{\varepsilon \in V} \varepsilon_{i}^{2} \varepsilon_{j}^{2}\right)+\frac{1}{t}\left(\begin{array}{l}
4 \\
1
\end{array}\right) \sum_{i, j=1}^{p} q_{i}^{3} q_{j}\left(\sum_{\varepsilon \in V} \varepsilon_{i}^{3} \varepsilon_{j}\right) \\
& +\frac{1}{t} \frac{4 !}{2 !} \sum_{i, j, k=1}^{p}{ }_{i} q_{i} q_{j} q_{k}^{2}\left(\sum_{\varepsilon \in V} \varepsilon_{i} \varepsilon_{j} \varepsilon_{k}^{2}\right)+\frac{1}{t} \sum_{i, j, k, l=1}^{p} q_{i} q_{j} q_{k} q_{l}\left(\sum_{\varepsilon \in V} \varepsilon_{i} \varepsilon_{j} \varepsilon_{k} \varepsilon_{l}\right),
\end{aligned}
$$

onde $\sum^{*}$ é a soma sobre todas as coordenadas duas a duas distintas. 
Sabemos que, para cada par de coordenadas $i$ e $j$ distintas,

$$
\left(\sum_{\varepsilon \in V} \varepsilon_{i}^{4}\right)=\left(\sum_{\varepsilon \in V} \varepsilon_{i}^{2} \varepsilon_{j}^{2}\right)=t
$$

e, pela prova da Proposição 37, como os $\varepsilon_{j}$ são dois a dois independentes, para cada coordenada $i, j, k$ duas a duas distintas, temos

$$
\left(\sum_{\varepsilon \in V} \varepsilon_{i} \varepsilon_{j} \varepsilon_{k}^{2}\right)=\left(\sum_{\varepsilon \in V} \varepsilon_{i}^{3} \varepsilon_{j}\right)=\left(\sum_{\varepsilon \in V} \varepsilon_{i} \varepsilon_{j}\right)=0 .
$$

Pela definição do conjunto $V$, para cada coordenada $i_{1}, i_{2}, i_{3}, i_{4}$ duas a duas distintas e para cada $\delta_{i} \in\{-1,1\}$ com $1 \leq i \leq 4$, exatamente $t / 16$ vetores em $V$ tem $\varepsilon_{i_{k}}=\delta_{k}$ com $1 \leq k \leq 4$. Assim,

$$
\left(\sum_{\varepsilon \in V} \varepsilon_{i} \varepsilon_{j} \varepsilon_{k} \varepsilon_{l}\right)=\left[\left(\begin{array}{l}
4 \\
0
\end{array}\right)+\left(\begin{array}{l}
4 \\
2
\end{array}\right)+\left(\begin{array}{l}
4 \\
4
\end{array}\right)\right] \frac{t}{16}+(-1)\left[\left(\begin{array}{l}
4 \\
1
\end{array}\right)+\left(\begin{array}{l}
4 \\
3
\end{array}\right)\right] \frac{t}{16}=0 .
$$

Sabendo que

$$
\left(\sum_{j=1}^{p} q_{j}^{2}\right)^{2}=\left(\sum_{j=1}^{p} q_{j}^{4}\right)+2\left(\sum_{1 \leq i<j \leq p} q_{i}^{2} q_{j}^{2}\right)
$$

temos

$$
\mathbb{E}\left([H(q)]^{4}\right)=\sum_{j=1}^{p} q_{j}^{4}+6 \sum_{1 \leq i<j \leq p} q_{i}^{2} q_{j}^{2} \leq 3\left(\sum_{j=1}^{p} q_{j}^{2}\right)^{2} \leq 3
$$

como queríamos.

O $M$-truncamento de $H(q)$, denotado por $H^{M}(q)$, é uma variável aleatória de $V$ nos reais definida como

$$
H^{M}(q)(\varepsilon)= \begin{cases}-M, & \text { se }[H(q)](\varepsilon)<-M \\ {[H(q)](\varepsilon),} & \text { se }|[H(q)](\varepsilon)| \leq M \\ M, & \text { se }[H(q)](\varepsilon)>M\end{cases}
$$

Proposição 39. Dado um vetor unitário $q \in \mathbb{R}^{p}$, o valor esperado de $\left[H^{M}(q)\right]^{2}$ é no máximo 1 . Demonstração. Basta observar que $H^{M}(q)(\varepsilon) \leq H(q)(\varepsilon)$ para todo $\varepsilon \in V$.

Queremos saber, em média, quanto $H(q)$ e $H^{M}(q)$ diferem. Para isso, recordemo-nos da Desigualdade de Markov.

Teorema 40 (Desigualdade de Markov). Seja X uma variável aleatória. Para todo real $t>0$, temos

$$
\mathbb{P}[|X| \geq \lambda] \leq \frac{\mathbb{E}\left[|X|^{t}\right]}{\lambda^{t}}
$$

para todo real $\lambda>0$.

Pela Desigualdade de Markov, para todo real $m>0$,

$$
\mathbb{P}[|H(q)| \geq m] \leq \frac{\mathbb{E}\left([H(q)]^{4}\right)}{m^{4}} \leq \frac{3}{m^{4}} .
$$

A desigualdade (3.7) será útil para o lema abaixo.

Lema 41. O valor esperado de $\left|H(q)-H^{M}(q)\right|^{2}$ satisfaz

$$
\mathbb{E}\left[\left|H(q)-H^{M}(q)\right|^{2}\right] \leq \frac{1}{M^{2}} .
$$


Demonstração. Note que

$$
H(q)(\varepsilon)-H^{M}(q)(\varepsilon)= \begin{cases}H(q)(\varepsilon)+M & \text { se } H(q)(\varepsilon)<-M \\ 0 & \text { se }|H(q)(\varepsilon)| \leq M \\ H(q)(\varepsilon)-M & \text { se } H(q)(\varepsilon)>M\end{cases}
$$

Logo,

$$
\left|H(q)(\varepsilon)-H^{M}(q)(\varepsilon)\right|= \begin{cases}0 & \text { se }|H(q)(\varepsilon)| \leq M \\ |H(q)(\varepsilon)|-M & \text { se }|H(q)(\varepsilon)|>M\end{cases}
$$

Por definição, o evento $\left[\left|H(q)-H^{M}(q)\right| \geq \sqrt{m}\right]$ é igual ao evento $[|H(q)| \geq M+\sqrt{m}]$. Portanto, pela desigualdade (3.7), temos que

$$
\begin{aligned}
\mathbb{E}\left[\left|H(q)-H^{M}(q)\right|^{2}\right] & =\int_{0}^{+\infty} \mathbb{P}\left[\left|H(q)-H^{M}(q)\right| \geq \sqrt{m}\right] \mathrm{d} m \\
& =\int_{0}^{+\infty} \mathbb{P}[|H(q)| \geq M+\sqrt{m}] \mathrm{d} m \\
& \leq \int_{0}^{+\infty} \frac{3}{(M+\sqrt{m})^{4}} \mathrm{~d} m \\
& =\frac{1}{M^{2}}
\end{aligned}
$$

como queríamos.

Cada variável aleatória $H(q)$ pode ser associada a um vetor $h(q)$ em $\mathbb{R}^{t}$ definido por $[h(q)](\varepsilon)=$ $[H(q)(\varepsilon)] / \sqrt{t}$. O $M$-truncamento $h^{M}(q)=H^{M}(q) / \sqrt{t}$ é definido analogamente.

Lema 42. Para cada vetor unitário $q \in \mathbb{R}^{p}$, o vetor $h(q) \in \mathbb{R}^{t}$ é unitário. A norma de $h^{M}(q)$ é no máximo 1 , e a norma de $h(q)-h^{M}(q)$ é no máximo $1 / M$. Se $q^{\prime} \in \mathbb{R}^{p}$ é um outro vetor unitário, então $\left\langle h(q), h\left(q^{\prime}\right)\right\rangle=\left\langle q, q^{\prime}\right\rangle$.

Demonstração. Seja $q$ um vetor unitário em $\mathbb{R}^{p}$. O vetor $h(q)$ é unitário pois

$$
\|h(q)\|_{2}^{2}=\sum_{\varepsilon \in V} h(q)(\varepsilon)^{2}=\frac{1}{t} \sum_{\varepsilon \in V} H(q)(\varepsilon)^{2}=\mathbb{E}\left[H(q)^{2}\right]=1 .
$$

Seja $M>0$ um real qualquer. A norma do vetor $h^{M}(q)$ é no máximo um pois

$$
\left\|h^{M}(q)\right\|_{2}^{2}=\sum_{\varepsilon \in V} h^{M}(q)(\varepsilon)^{2}=\frac{1}{t} \sum_{\varepsilon \in V} H^{M}(q)(\varepsilon)^{2}=\mathbb{E}\left[H^{M}(q)^{2}\right] \leq 1 .
$$

A norma de $h(q)-h^{M}(q)$ é no máximo $1 / M$ pois

$$
\begin{aligned}
\left\|h(q)-h^{M}(q)\right\|_{2}^{2}=\sum_{\varepsilon \in V}\left|h(q)(\varepsilon)-h^{M}(q)(\varepsilon)\right|^{2} & \left.=\frac{1}{t} \sum_{\varepsilon \in V} \mid H(q)(\varepsilon)-H^{M}(q)\right)\left.(\varepsilon)\right|^{2} \\
& =\mathbb{E}\left[\left|H(q)-H^{M}(q)\right|^{2}\right] \leq \frac{1}{M^{2}}
\end{aligned}
$$

Seja $q^{\prime}$ um vetor unitário em $\mathbb{R}^{p}$. Temos

$$
\begin{aligned}
\left\langle h(q), h\left(q^{\prime}\right)\right\rangle=\sum_{\varepsilon \in V} h(q)(\varepsilon) h\left(q^{\prime}\right)(\varepsilon) & =\frac{1}{t} \sum_{\varepsilon \in V} H(q)(\varepsilon) H\left(q^{\prime}\right)(\varepsilon) \\
& =\mathbb{E}\left[H(q) H\left(q^{\prime}\right)\right] \\
& =\left\langle q, q^{\prime}\right\rangle
\end{aligned}
$$


como queríamos.

Mostremos o Teorema 36. Enunciaremos o teorema novamente abaixo.

Teorema 43. Existe um algoritmo em tempo polinomial que produz, para toda matriz $A=\left(a_{i j}\right)$ do tipo $m \times n$, inteiros $\left\{\varepsilon_{i}\right\}_{i=1}^{m},\left\{\delta_{j}\right\}_{j=1}^{n} \subseteq\{-1,1\}$ tais que

$$
\sum_{i j} a_{i j} \varepsilon_{i} \delta_{j} \geq 0.03 \operatorname{SDP}(A)
$$

Demonstração. Sejam $A$ uma matriz do tipo $m \times n$ e $\delta>0$ um real qualquer. Sejam $\left\{u_{i}\right\}_{i=1}^{m},\left\{v_{j}\right\}_{j=1}^{n}$ uma solução viável do programa semidefinido $\operatorname{SDP}(A)$ satisfazendo

$$
\sum_{i j} a_{i j}\left\langle u_{i}, v_{j}\right\rangle \geq \operatorname{SDP}(A)-\delta
$$

Como $\left\langle h(q), h\left(q^{\prime}\right)\right\rangle=\left\langle q, q^{\prime}\right\rangle$ para todo vetor unitário $q, q^{\prime}$, segue que

$$
\begin{aligned}
\operatorname{SDP}(A)-\delta \leq & \sum_{i j} a_{i j}\left\langle u_{i}, v_{j}\right\rangle \\
= & \sum_{i j} a_{i j}\left\langle h\left(u_{i}\right), h\left(v_{j}\right)\right\rangle \\
= & \sum_{i j} a_{i j}\left\langle h^{M}\left(u_{i}\right), h^{M}\left(v_{j}\right)\right\rangle+\sum_{i j} a_{i j}\left\langle h\left(u_{i}\right)-h^{M}\left(u_{i}\right), h^{M}\left(v_{j}\right)\right\rangle+ \\
& \sum_{i j} a_{i j}\left\langle h\left(u_{i}\right), h\left(v_{j}\right)-h^{M}\left(v_{j}\right)\right\rangle .
\end{aligned}
$$

Pela convexidade do programa, e como a norma dos vetores $h^{M}\left(v_{j}\right)$ e $h\left(u_{i}\right)$ é no máximo 1 , e a norma de cada vetor $h\left(u_{i}\right)-h^{M}\left(u_{i}\right)$ e de cada vetor $h\left(v_{j}\right)-h^{M}\left(v_{j}\right)$ é no máximo $1 / M$, temos

$$
\begin{aligned}
& \sum_{i j} a_{i j}\left\langle h\left(u_{i}\right)-h^{M}\left(u_{i}\right), h^{M}\left(v_{j}\right)\right\rangle+\sum_{i j} a_{i j}\left\langle h\left(u_{i}\right), h\left(v_{j}\right)-h^{M}\left(v_{j}\right)\right\rangle \\
& =\frac{1}{M} \sum_{i j} a_{i j}\left\langle M\left(h\left(u_{i}\right)-h^{M}\left(u_{i}\right)\right), h^{M}\left(v_{j}\right)\right\rangle+\frac{1}{M} \sum_{i j} a_{i j}\left\langle h\left(u_{i}\right), M\left(h\left(v_{j}\right)-h^{M}\left(v_{j}\right)\right)\right\rangle \\
& \leq \frac{2}{M} \operatorname{SDP}(A) .
\end{aligned}
$$

Portanto,

$$
\begin{aligned}
\operatorname{SDP}(A)\left(1-\frac{2}{M}\right)-\delta & \leq \sum_{i j} a_{i j}\left\langle h^{M}\left(u_{i}\right), h^{M}\left(v_{j}\right)\right\rangle \\
& =\sum_{i j} a_{i j} \sum_{\varepsilon \in V} h^{M}\left(u_{i}\right)(\varepsilon) h^{M}\left(v_{j}\right)(\varepsilon) \\
& =\sum_{\varepsilon \in V} \sum_{i j} a_{i j} h^{M}\left(u_{i}\right)(\varepsilon) h^{M}\left(v_{j}\right)(\varepsilon) .
\end{aligned}
$$

Logo, existe $\varepsilon \in V$ tal que

$$
\sum_{i j} a_{i j} h^{M}\left(u_{i}\right)(\varepsilon) h^{M}\left(v_{j}\right)(\varepsilon) \geq \frac{1}{t}\left[\operatorname{SDP}(A)\left(1-\frac{2}{M}\right)-\delta\right] .
$$

Assim,

$$
\sum_{i j} a_{i j} H^{M}\left(u_{i}\right)(\varepsilon) H^{M}\left(v_{j}\right)(\varepsilon) \geq \operatorname{SDP}(A)\left(1-\frac{2}{M}\right)-\delta .
$$


Tomando $M=3$, e definindo $\varepsilon_{i}=H^{M}\left(u_{i}\right)(\varepsilon) / M$ e $\delta_{j}=H^{M}\left(v_{j}\right)(\varepsilon) / M$, temos que $\varepsilon_{i}$ e $\delta_{j}$ são reais satisfazendo

$$
\sum_{i j} a_{i j} \varepsilon_{i} \delta_{j} \geq \operatorname{SDP}(A)\left(\frac{M-2}{M^{3}}\right)-\frac{\delta}{M^{2}}=\frac{\operatorname{SDP}(A)}{27}-\frac{\delta}{9} .
$$

Fixando todo os $\varepsilon_{i}, \delta_{j}$ menos, digamos $\varepsilon_{1}$, temos que o lado esquerdo da desigualdade (3.8) é uma função linear em $\varepsilon_{1}$, e logo podemos transladar $\varepsilon_{1}$ a -1 ou 1 , sem diminuir o valor da soma. Procedendo para cada outra variável, obtemos $\varepsilon_{i}, \delta_{j} \in\{-1,1\}$ satisfazendo a desigualdade (3.8). Como $1 / 27 \geq 0.03$ e tomando $\delta$ arbitrariamente pequeno, obtemos a desigualdade desejada.

\subsection{Melhorando a garantia de desempenho}

Na Seção 3.2, obtivemos uma aproximação da norma do corte com garantia de desempenho igual a $\rho=0.03$. Vamos melhorar essa garantia de desempenho para $\rho=0.27$ construindo um algoritmo que se baseia na prova do Teorema de Grothendieck por Rietz [Rie74].

Definimos a função sinal sign : $\mathbb{R} \rightarrow\{-1,0,1\}$ por

$$
\operatorname{sign}(x)=\left\{\begin{array}{l}
-1, \text { se } x<0 \\
0, \text { se } x=0 \\
1, \text { se } x>0
\end{array}\right.
$$

Sejam $p=m+n$ e $g_{1}, \ldots, g_{p}$ variáveis aleatórias normais independentes, e considere o vetor aleatório normal $G=\left(g_{1}, \ldots, g_{p}\right)$. Vamos precisar do seguinte lema.

Lema 44. Seja $G=\left(g_{1}, \ldots, g_{p}\right)$ um vetor aleatório normal, e sejam b,c vetores unitários em $\mathbb{R}^{p}$. Temos a seguinte identidade:

$$
\frac{\pi}{2} \mathbb{E}[\operatorname{sign}\langle b, G\rangle \operatorname{sign}\langle c, G\rangle]=\langle b, c\rangle+\mathbb{E}\left\{\left[\langle b, G\rangle-\sqrt{\frac{\pi}{2}} \operatorname{sign}\langle b, G\rangle\right]\left[\langle c, G\rangle-\sqrt{\frac{\pi}{2}} \operatorname{sign}\langle c, G\rangle\right]\right\} .
$$

Demonstração. Mostremos primeiramente que

$$
\mathbb{E}[\langle b, G\rangle\langle c, G\rangle]=\langle b, c\rangle .
$$

Sabendo que $\mathbb{E}\left[g_{i} g_{j}\right]=\delta_{i j}$, e escrevendo $b=\left(b_{1}, \ldots, b_{p}\right)$ e $c=\left(c_{1}, \ldots, c_{p}\right)$, temos

$$
\mathbb{E}[\langle b, G\rangle\langle c, G\rangle]=\mathbb{E}\left[\sum_{i=1}^{p} b_{i} g_{i} \sum_{j=1}^{p} c_{j} g_{j}\right]=\sum_{i, j=1}^{p} b_{i} c_{j} \mathbb{E}\left[g_{i} g_{j}\right]=\sum_{i=1}^{p} b_{i} c_{i}=\langle b, c\rangle .
$$

Para calcular $\mathbb{E}[\langle b, G\rangle \operatorname{sign}\langle c, G\rangle]$ podemos assumir, por invariância rotacional, que $c=(1,0, \ldots, 0)$ e $b=\left(b_{1}, b_{2}, 0, \ldots, 0\right)$. Assim

$$
\begin{aligned}
\mathbb{E}[\langle b, G\rangle \operatorname{sign}\langle c, G\rangle] & =\mathbb{E}\left[\left(b_{1} g_{1}+b_{2} g_{2}\right) \operatorname{sign}\left(g_{1}\right)\right] \\
& =\mathbb{E}\left[b_{1} g_{1} \operatorname{sign}\left(g_{1}\right)\right]+\mathbb{E}\left[b_{2} g_{2}\right] \mathbb{E}\left[\operatorname{sign}\left(g_{1}\right)\right] \\
& =\mathbb{E}\left[b_{1} g_{1} \operatorname{sign}\left(g_{1}\right)\right] \\
& =2 b_{1} \int_{0}^{\infty} \frac{1}{\sqrt{2 \pi}} x e^{-x^{2} / 2} \mathrm{~d} x \\
& =\sqrt{\frac{2}{\pi}} b_{1} \\
& =\sqrt{\frac{2}{\pi}}\langle b, c\rangle,
\end{aligned}
$$

onde na segunda igualdade usamos que as variáveis aleatórias $g_{2}$ e $\operatorname{sign}\left(g_{1}\right)$ são independentes 
e $\mathbb{E}\left[\operatorname{sign}\left(g_{1}\right)\right]=0$, e na quarta e na quinta igualdade usamos que

$$
\mathbb{E}\left[g_{1} \operatorname{sign}\left(g_{1}\right)\right]=\int_{-\infty}^{+\infty} \frac{|x|}{\sqrt{2 \pi}} e^{-x^{2} / 2} \mathrm{~d} x=2 \int_{0}^{+\infty} \frac{x}{\sqrt{2 \pi}} e^{-x^{2} / 2} \mathrm{~d} x=\frac{2}{\sqrt{2 \pi}} \int_{0}^{+\infty} x e^{-x^{2} / 2} \mathrm{~d} x=\frac{2}{\sqrt{2 \pi}} .
$$

Portanto,

$$
\begin{aligned}
& \mathbb{E}\left\{\left[\langle b, G\rangle-\sqrt{\frac{\pi}{2}} \operatorname{sign}\langle b, G\rangle\right]\left[\langle c, G\rangle-\sqrt{\frac{\pi}{2}} \operatorname{sign}\langle c, G\rangle\right]\right\} \\
& =\mathbb{E}[\langle b, G\rangle\langle c, G\rangle]-\sqrt{\frac{\pi}{2}} \mathbb{E}[\langle b, G\rangle \operatorname{sign}\langle c, G\rangle]-\sqrt{\frac{\pi}{2}} \mathbb{E}[\langle c, G\rangle \operatorname{sign}\langle b, G\rangle]+\frac{\pi}{2} \mathbb{E}[\operatorname{sign}\langle b, G\rangle \operatorname{sign}\langle c, G\rangle] \\
& =-\langle b, c\rangle+\frac{\pi}{2} \mathbb{E}[\operatorname{sign}\langle b, G\rangle \operatorname{sign}\langle c, G\rangle],
\end{aligned}
$$

como queríamos.

O Lema 44 é essencial para melhorar a garantia de desempenho para a aproximação da solução do programa (3.5).

Teorema 45 (Alon-Naor, 2006). Existe um algoritmo em tempo polinomial que produz, para toda matriz $A=\left(a_{i j}\right)$ do tipo $m \times n$, inteiros $\left\{\varepsilon_{i}\right\}_{i=1}^{m},\left\{\delta_{j}\right\}_{j=1}^{n} \subseteq\{-1,1\}$ tais que

$$
\sum_{i j} a_{i j} \varepsilon_{i} \delta_{j} \geq\left(\frac{4}{\pi}-1\right)\|A\|_{\infty \rightarrow 1} .
$$

Demonstração. Sejam $\left\{u_{i}\right\}_{i=1}^{m},\left\{v_{j}\right\}_{j=1}^{n} \subseteq S^{m+n-1}$ soluções ótimas do programa (3.5). Substituindo $u_{i}$ e $v_{j}$ na identidade (3.9) do Lema 44, multiplicando por $a_{i j}$ e somando todos os termos, temos

$$
\begin{aligned}
& \frac{\pi}{2} \mathbb{E}\left[\sum_{i j} a_{i j} \operatorname{sign}\left\langle u_{i}, G\right\rangle \operatorname{sign}\left\langle v_{j}, G\right\rangle\right] \\
& =\operatorname{SDP}(A)+\sum_{i j} a_{i j} \mathbb{E}\left\{\left[\left\langle u_{i}, G\right\rangle-\sqrt{\frac{\pi}{2}} \operatorname{sign}\left\langle u_{i}, G\right\rangle\right]\left[\left\langle v_{j}, G\right\rangle-\sqrt{\frac{\pi}{2}} \operatorname{sign}\left\langle v_{j}, G\right\rangle\right]\right\} .
\end{aligned}
$$

Para cada vetor unitário $b$, seja $z(b, G)=\langle b, G\rangle-\sqrt{\pi / 2} \operatorname{sign}\langle b, G\rangle$. Note que $z(b, G)$ é uma variável aleatória para todo vetor unitário $b$.

Para cada duas variáveis aleatórias $X, Y$, a operação $\langle X, Y\rangle=\mathbb{E}[X Y]$ define um produto interno em um espaço de variáveis aleatórias. Assim, para todo vetor unitário $b$,

$$
\begin{aligned}
\langle z(b, G), z(b, G)\rangle & =\mathbb{E}\left\{\left[\langle b, G\rangle-\sqrt{\frac{\pi}{2}} \operatorname{sign}\langle b, G\rangle\right]^{2}\right\} \\
& =\frac{\pi}{2} \mathbb{E}\left[(\operatorname{sign}\langle b, G\rangle)^{2}\right]-\|b\|_{2}^{2} \\
& =\frac{\pi}{2}-1 .
\end{aligned}
$$

Como temos um produto interno em um espaço de Hilbert (o espaço de variáveis aleatórias) e o programa (3.4) foi definido sobre qualquer espaço de Hilbert, temos que para quaisquer variáveis aleatórias $X, Y$ com norma $\sqrt{\pi / 2-1}$, temos

$$
\sum_{i j} a_{i j}\langle X, Y\rangle \leq\left(\frac{\pi}{2}-1\right) \operatorname{SDP}(A) .
$$


Em particular, tomando $X=-z\left(u_{i}, G\right)$ e $Y=z\left(v_{j}, G\right)$,

$$
\sum_{i j} a_{i j}\left\langle-z\left(u_{i}, G\right), z\left(v_{j}, G\right)\right\rangle \leq\left(\frac{\pi}{2}-1\right) \operatorname{SDP}(A) .
$$

Multiplicando ambos os lados por -1, e pela definição do produto interno,

$$
\sum_{i j} a_{i j} \mathbb{E}\left[z\left(u_{i}, G\right) z\left(v_{j}, G\right)\right] \geq\left(1-\frac{\pi}{2}\right) \operatorname{SDP}(A) .
$$

Substituindo agora a identidade (3.10), obtemos

$$
\frac{\pi}{2} \mathbb{E}\left[\sum_{i j} a_{i j} \operatorname{sign}\left\langle u_{i}, G\right\rangle \operatorname{sign}\left\langle v_{j}, G\right\rangle\right] \geq\left(2-\frac{\pi}{2}\right) \operatorname{SDP}(A) .
$$

Sabendo que $\operatorname{SDP}(A) \geq\|A\|_{\infty \rightarrow 1}$ e tomando $\varepsilon_{i}=\operatorname{sign}\left\langle u_{i}, G\right\rangle$ e $\delta_{j}=\operatorname{sign}\left\langle v_{j}, G\right\rangle$, o resultado segue. 


\section{Capítulo 4}

\section{Desigualdade de Grothendieck}

A Desigualdade de Grothendieck é uma importante desigualdade da teoria dos espaços de Banach. Foi provada por Alexander Grothendieck em 1956 e publicada no artigo "Résumé de la théorie métrique des produits tensoriels topologiques" [Gro53].

Como o artigo [Gro53] foi escrito em francês e foi publicado em uma revista brasileira de pouca circulação, foi praticamente ignorado pelos pesquisadores na época, apesar de o artigo ter sido revisado em Math Reviews por Dvoretzky.

Em 1968, Lindenstrauss e Pełczýnski, em [LP68], descobriram vários resultados utilizando a Desigualdade de Grothendieck, solucionando até problemas em aberto que foram propostos após a publicação do artigo de Grothendieck.

A Desigualdade de Grothendieck possui várias aplicações, tanto na Teoria dos Espaços de Banach quanto em outras áreas, como em $C^{*}$-Álgebra [Pis86], Mecânica Quântica [Tsi85], Teoria da Informação Quântica [PGWP+08] e Ciência da Computação [AN06]. Uma boa referência sobre o assunto é a recente resenha de Pisier [Pis12]. A pesquisa de Khot e Naor [KN12] dedica-se às aplicações da Desigualdade de Grothendieck exclusivamente na Ciência da Computação.

\subsection{Argumento de Krivine}

Enunciemos a versão elementar do resultado de Grothendieck, reformulado por Lindenstrauss e Pełczýnski.

Teorema 46 (Grothendieck, 1953). Seja $\left(a_{i j}\right)$ uma matriz do tipo $m \times n$ tal que para todo intei$\operatorname{ros}\left\{\varepsilon_{i}\right\}_{i=1}^{m},\left\{\delta_{j}\right\}_{j=1}^{n}$ em $\{-1,1\}$ temos

$$
\left|\sum_{i j} a_{i j} \varepsilon_{i} \delta_{j}\right| \leq M,
$$

para algum $M$ real positivo. Então para todo espaço de Hilbert $H$, existe um real positivo $K$ que não depende da matriz e nem de $m$ e $n$, tal que, para todos os vetores unitários $\left\{u_{i}\right\}_{i=1}^{m},\left\{v_{j}\right\}_{j=1}^{n}$ em $H$, temos

$$
\left|\sum_{i j} a_{i j}\left\langle u_{i}, v_{j}\right\rangle\right| \leq K M .
$$

O ínfimo da constante $K$ sobre todo espaço de Hilbert e $m, n$ inteiros positivos é denotado por $K_{G}$, e chamado de constante de Grothendieck.

A seguir, veremos que podemos obter uma relação entre $\operatorname{SDP}(A)$ e $\|A\|_{\infty \rightarrow 1}$ a partir do Teorema 46.

Corolário 47 (Desigualdade de Grothendieck). Para toda matriz A do tipo $m \times n$, temos

$$
\operatorname{SDP}(A) \leq K_{G}\|A\|_{\infty \rightarrow 1} .
$$


Demonstração. Basta tomar $M=\|A\|_{\infty \rightarrow 1}$ na desigualdade (4.1), tomar $H=\mathbb{R}^{m+n}$ e calcular o máximo de $\left|\sum_{i j} a_{i j}\left\langle u_{i}, v_{j}\right\rangle\right|$ sobre todos os vetores unitários $\left\{u_{i}\right\}_{i=1}^{m},\left\{v_{j}\right\}_{j=1}^{n}$ em $\mathbb{R}^{m+n}$ na desigualdade (4.2).

A desigualdade (4.3) é conhecida por Desigualdade de Grothendieck. Sabendo que $\|A\|_{\infty \rightarrow 1} \leq$ $\operatorname{SDP}(A)$, e pelo Corolário 47, temos

$$
\|A\|_{\infty \rightarrow 1} \leq \operatorname{SDP}(A) \leq K_{G}\|A\|_{\infty \rightarrow 1} .
$$

Calcular o valor exato da constante de Grothendieck é ainda um problema em aberto. Em [Gro53] Grothendieck apresentou a seguinte cota:

$$
1,570 \ldots=\frac{\pi}{2} \leq K_{G} \leq \operatorname{senh}\left(\frac{\pi}{2}\right)=2.301 \ldots
$$

Após algum progresso obtido por Rietz [Rie74], Krivine [Kri77] mostrou que

$$
K_{G} \leq \frac{\pi}{2 \log (1+\sqrt{2})}=1.782 \ldots
$$

e conjecturou que esse é o valor exato. Sua conjectura foi desprovada por Braverman, Makarychev, Makarychev e Naor [BMMN11]. A cota de Krivine é a melhor conhecida até hoje.

A melhor cota inferior para $K_{G}$ foi apresentada por Reeds em um artigo não publicado [Ree91], que provou que

$$
K_{G} \geq \frac{\pi}{2} e^{\eta_{0}^{2}}=1.676 \ldots
$$

onde $\eta_{0}=0.25573 \ldots$ é a solução única da equação

$$
1-2 \sqrt{\frac{2}{\pi}} \int_{0}^{\eta} e^{-z^{2} / 2} d z=\frac{2}{\pi} e^{-\eta^{2}}
$$

Alon e Naor apresentaram em [AN06] uma aplicação da Desigualdade de Grothendieck na teoria dos grafos. Eles encontraram um algoritmo de aproximação em tempo polinomial para a norma do corte. Ademais, provaram que o problema de aproximar a norma do corte de uma matriz real é MAX SNP-difícil. O algoritmo encontra dois subconjuntos $S_{0} \subseteq[\mathrm{m}]$ e $T_{0} \subseteq[n]$ tais que $\left|\sum_{i \in S_{0}, j \in T_{0}} a_{i j}\right| \geq$ $K_{G}^{-1}\|A\|_{c u t}$.

O Teorema de Raghavendra e Steurer [RS09] afirma que existe uma relação entre a constante de Grothendieck $K_{G}$ e o problema $P=N P$, a saber:

Teorema 48 (Raghavendra-Steurer, 2009). Assumindo a Conjectura do Jogo Único, para todo $0<$ $K<K_{G}$ e A matriz do tipo $m \times n$, é $N P$-difícil calcular um real positivo $q$ tal que $K^{-1} q \leq\|A\|_{\infty \rightarrow 1}$.

Recordemos que a Conjectura do Jogo Único afirma que um certo problema computacional conhecido como Jogo Único é inaproximável. Recomendamos o artigo [Kho02] para mais informações.

Em 1977, Krivine mostrou [Kri77] que a constante de Grothendieck é no máximo $\pi /(2 \log (1+$ $\sqrt{2}$ ). A prova ficou conhecida como Argumento de Krivine. Ademais, Krivine conjecturou que esse é o valor exato da constante. Essa conjectura foi reformulada repetidamente em diversas publicações posteriores; veja por exemplo [Pis78], [Kön01] e [CPTW04].

Vamos apresentar uma prova da Desigualdade de Grothendieck devido ao Alon e Naor, que é uma versão simplificada do Argumento de Krivine.

Para provar a Desigualdade de Grothendieck será necessário o lema a seguir, conhecido como Identidade de Grothendieck.

Lema 49 (Grothendieck, 1953). Sejam $x$, y vetores unitários em $\mathbb{R}^{n}$ e r um vetor unitário escolhido uniformemente ao acaso de acordo com a distribuição de probabilidade invariante por rotação sobre a esfera unitária. Temos

$$
\mathbb{E}[\operatorname{sign}\langle x, r\rangle \operatorname{sign}\langle y, r\rangle]=\frac{2}{\pi} \operatorname{arcsen}\langle x, y\rangle
$$


Demonstração. Por definição, a esperança pode ser calculada como

$$
\mathbb{E}[\operatorname{sign}\langle x, r\rangle \operatorname{sign}\langle y, r\rangle]=\mathbb{P}[\operatorname{sign}\langle x, r\rangle=\operatorname{sign}\langle y, r\rangle]-\mathbb{P}[\operatorname{sign}\langle x, r\rangle \neq \operatorname{sign}\langle y, r\rangle] .
$$

Como

$$
\mathbb{P}[\operatorname{sign}\langle x, r\rangle=\operatorname{sign}\langle y, r\rangle]=1-\mathbb{P}[\operatorname{sign}\langle x, r\rangle \neq \operatorname{sign}\langle y, r\rangle],
$$

basta calcular a probabilidade dos sinais de $\langle x, r\rangle$ e $\langle y, r\rangle$ serem diferentes.

Por simetria, temos $\mathbb{P}[\operatorname{sign}\langle x, r\rangle \neq \operatorname{sign}\langle y, r\rangle]=2 \mathbb{P}[\operatorname{sign}\langle x, r\rangle \geq 0$ e $\operatorname{sign}\langle y, r\rangle<0]$. O conjunto $\left\{r \in S^{n-1}: \operatorname{sign}\langle x, r\rangle \geq 0\right.$ e $\left.\operatorname{sign}\langle y, r\rangle<0\right\}$ corresponde à interseção de dois semi-espaços cujo ângulo diedral é $\theta=\arccos \langle x, y\rangle$, e por simetria da esfera, o conjunto tem medida igual a $\theta / 2 \pi$ vezes a medida da esfera unitária. Logo

$$
\mathbb{P}[\operatorname{sign}\langle x, r\rangle \geq 0 \text { e } \operatorname{sign}\langle y, r\rangle<0]=\frac{\arccos \langle x, y\rangle}{2 \pi} .
$$

Portanto

$$
\begin{aligned}
\mathbb{E}[\operatorname{sign}\langle x, r\rangle \operatorname{sign}\langle y, r\rangle] & =1-2 \mathbb{P}[\operatorname{sign}\langle x, r\rangle \neq \operatorname{sign}\langle y, r\rangle] \\
& =1-2\left(2 \frac{\arccos \langle x, y\rangle}{2 \pi}\right) \\
& =1-\frac{2}{\pi} \arccos \langle x, y\rangle \\
& =\frac{2}{\pi} \operatorname{arcsen}\langle x, y\rangle .
\end{aligned}
$$

Usamos a identidade

$$
\operatorname{arcsen} t+\arccos t=\frac{\pi}{2}
$$

para obter a igualdade desejada.

Sejam $v=\left(v_{1}, \ldots, v_{m}\right) \in \mathbb{R}^{m}$ e $w=\left(w_{1}, \ldots, w_{n}\right) \in \mathbb{R}^{n}$. O produto tensorial é o vetor em $\mathbb{R}^{m n}$ definido como segue,

$$
v \otimes w=\left(v_{1} w_{1}, \ldots, v_{1} w_{n}, v_{2} w_{1}, \ldots, v_{m} w_{n}\right)
$$

Note que

$$
\left\langle u_{1} \otimes v_{1}, u_{2} \otimes v_{2}\right\rangle=\left\langle u_{1}, u_{2}\right\rangle\left\langle v_{1}, v_{2}\right\rangle
$$

para todo $u_{1}, u_{2} \in \mathbb{R}^{m}$ e $v_{1}, v_{2} \in \mathbb{R}^{n}$. De fato, sejam $u_{i}=\left(u_{i}^{1}, \ldots, u_{i}^{m}\right)$ e $v_{i}=\left(v_{i}^{1}, \ldots, v_{i}^{n}\right)$ com $i=$ 1,2 .

$$
\left\langle u_{1} \otimes v_{1}, u_{2} \otimes v_{2}\right\rangle=\sum_{i j} u_{1}^{i} v_{1}^{j} u_{2}^{i} v_{2}^{j}=\left(\sum_{i=1}^{m} u_{1}^{i} u_{2}^{i}\right)\left(\sum_{j=1}^{n} v_{1}^{j} v_{2}^{j}\right)=\left\langle u_{1}, u_{2}\right\rangle\left\langle v_{1}, v_{2}\right\rangle .
$$

Lema 50. Sejam $u_{1}, \ldots, u_{m}$ e $v_{1}, \ldots, v_{n}$ vetores unitários em $\mathbb{R}^{m+n}$. Existem vetores unitários $\left\{u_{i}^{\prime}\right\}_{i=1}^{m}$ e $\left\{v_{j}^{\prime}\right\}_{j=1}^{n}$ em um espaço de Hilbert $H$ tais que se $r$ é um vetor de $H$ escolhido uniformemente ao acaso, então

$$
\frac{\pi}{2} \mathbb{E}\left[\operatorname{sign}\left\langle u_{i}^{\prime}, r\right\rangle \operatorname{sign}\left\langle v_{j}^{\prime}, r\right\rangle\right]=c\left\langle u_{i}, v_{j}\right\rangle
$$

para todo $1 \leq i \leq m e 1 \leq j \leq n$, onde $c=\log (1+\sqrt{2})=\operatorname{senh}^{-1}(1)$.

Demonstração. Sejam $u, v \in \mathbb{R}^{m+n}$. Por Expansão de Taylor,

$$
\operatorname{sen}(c\langle u, v\rangle)=\sum_{k=0}^{\infty}(-1)^{k} \frac{c^{2 k+1}}{(2 k+1) !}\langle u, v\rangle^{2 k+1} .
$$

Para todo vetor $w$ e inteiro positivo $j$, denotemos por $w^{\otimes j}$ a $j$-ésima potência tensorial $w \otimes \ldots \otimes w$. 
Pela identidade (4.6), temos $\langle u, v\rangle^{n}=\left\langle u^{\otimes n}, v^{\otimes n}\right\rangle$. Dessa forma,

$$
\operatorname{sen}(c\langle u, v\rangle)=\sum_{k=0}^{\infty}(-1)^{k} \frac{c^{2 k+1}}{(2 k+1) !}\left\langle u^{\otimes(2 k+1)}, v^{\otimes(2 k+1)}\right\rangle .
$$

Seja o espaço de Hilbert $H=\bigoplus_{k=0}^{\infty}\left(\mathbb{R}^{m+n}\right)^{\otimes(2 k+1)}$ munido do produto interno dado por

$$
\langle f, g\rangle=\sum_{k=0}^{\infty}\left\langle f_{k}, g_{k}\right\rangle,
$$

onde $f_{k}, g_{k} \in\left(\mathbb{R}^{m+n}\right)^{\otimes(2 k+1)}$.

Considere os seguintes vetores no espaço $H$ cujas $k$-ésimas coordenadas são dadas por

$$
\begin{aligned}
T(u)_{k} & =(-1)^{k} \sqrt{\frac{c^{2 k+1}}{(2 k+1) !}} u^{\otimes(2 k+1)} \\
S(v)_{k} & =\sqrt{\frac{c^{2 k+1}}{(2 k+1) !}} v^{\otimes(2 k+1)} .
\end{aligned}
$$

A equação (4.8) pode ser expressa como $\operatorname{sen}(c\langle u, v\rangle)=\langle T(u), S(v)\rangle$, que implica

$$
c\langle u, v\rangle=\operatorname{arcsen}\langle T(u), S(v)\rangle .
$$

Note que

$$
\begin{aligned}
& \|T(u)\|^{2}=\langle T(u), T(u)\rangle=\sum_{k=0}^{\infty} \frac{c^{2 k+1}}{(2 k+1) !}\left(\|u\|^{2}\right)^{2 k+1}=\operatorname{senh}\left(c\|u\|^{2}\right) \\
& \|S(v)\|^{2}=\langle S(v), S(v)\rangle=\sum_{k=0}^{\infty} \frac{c^{2 k+1}}{(2 k+1) !}\left(\|v\|^{2}\right)^{2 k+1}=\operatorname{senh}\left(c\|v\|^{2}\right) .
\end{aligned}
$$

Dados $\left\{u_{i}\right\}_{i=1}^{m}$ e $\left\{v_{j}\right\}_{j=1}^{n}$ vetores unitários, definimos $u_{i}^{\prime}=T\left(u_{i}\right)$ e $v_{j}^{\prime}=S\left(v_{j}\right)$. Note que tais vetores são unitários, já que $c=\operatorname{senh}^{-1}(1)$.

Seja $r$ um vetor aleatório escolhido uniformemente ao acaso sobre a esfera unitária. Pelo lema (49),

$$
\frac{\pi}{2} \mathbb{E}\left[\operatorname{sign}\left\langle T\left(u_{i}\right), r\right\rangle \operatorname{sign}\left\langle S\left(v_{j}\right), r\right\rangle\right]=\operatorname{arcsen}\left\langle T\left(u_{i}\right), S\left(v_{j}\right)\right\rangle=c\left\langle u_{i}, v_{j}\right\rangle,
$$

como queríamos.

Note que o conjunto dos vetores unitários $\left\{u_{i}^{\prime}\right\}_{i=1}^{m}$ e $\left\{v_{j}^{\prime}\right\}_{j=1}^{n}$ do espaço de Hilbert $H$ no Lema 50 geram um espaço isométrico a $\mathbb{R}^{m+n}$.

Teorema 51 (Krivine, 1977). Para toda matriz A do tipo $m \times n$, temos

$$
\operatorname{SDP}(A) \leq \frac{\pi}{2 \log (1+\sqrt{2})}\|A\|_{\infty \rightarrow 1}
$$

Demonstração. Seja $A$ uma matriz do tipo $m \times n$ e $\delta>0$. Existem vetores unitários $\left\{u_{i}\right\}_{i=1}^{m},\left\{v_{j}\right\}_{j=1}^{n}$ tais que

$$
\sum_{i j} a_{i j}\left\langle u_{i}, v_{j}\right\rangle \geq \operatorname{SDP}(A)-\delta
$$

Pelo Lema 50, existem vetores unitários $u_{1}^{\prime}, \ldots, u_{m}^{\prime}, v_{1}^{\prime}, \ldots, v_{n}^{\prime}$ satisfazendo a identidade (4.7).

Escolha um vetor aleatório $r \in S^{m+n-1}$ de acordo com a distribuição de probabilidade invariante 
por rotação sobre a esfera unitária. Sejam $\varepsilon_{i}=\operatorname{sign}\left\langle u_{i}^{\prime}, r\right\rangle$ e $\delta_{j}=\operatorname{sign}\left\langle v_{j}^{\prime}, r\right\rangle$. Temos

$$
\begin{aligned}
\|A\|_{\infty \rightarrow 1} \geq \mathbb{E}\left[\sum_{i j} a_{i j} \varepsilon_{i} \delta_{j}\right] & =\sum_{i j} a_{i j} \mathbb{E}\left[\operatorname{sign}\left\langle u_{i}^{\prime}, r\right\rangle \operatorname{sign}\left\langle v_{j}^{\prime}, r\right\rangle\right] \\
& =\sum_{i j} a_{i j} \frac{2}{\pi} c\left\langle u_{i}, v_{j}\right\rangle \\
& =\frac{2}{\pi} c(\operatorname{SDP}(A)-\delta) .
\end{aligned}
$$

Fazendo $\delta \rightarrow 0$, obtemos $\operatorname{SDP}(A) \leq \frac{\pi}{2 \log (1+\sqrt{2})}\|A\|_{\infty \rightarrow 1}$, como queríamos.

\subsection{Aproximação da norma de corte via Desigualdade de Grothen- dieck}

O Problema da Norma de Corte consiste em calcular a norma de corte de qualquer matriz. Em [AN06], Alon e Naor encontraram um algoritmo de aproximação para o Problema da Norma de Corte baseado na prova de Krivine. Ademais, esse algoritmo de aproximação possui a garantia de desempenho igual a $K_{G}^{-1}$. Apresentaremos nesta seção a construção desse algoritmo.

Pelo Lema 34, temos que para toda matriz $A$ do tipo $m \times n$, vale $\|A\|_{\infty \rightarrow 1} \leq 4\|A\|_{c u t}$. Veremos agora qual é a condição necessária para que ocorra a igualdade.

Lema 52. Seja A uma matriz do tipo $m \times n$. Se a soma de cada linha e de cada coluna é igual a zero, então

$$
\|A\|_{c u t}=\frac{1}{4}\|A\|_{\infty \rightarrow 1}
$$

Demonstração. Pelo Lema 34, vale que $\|A\|_{c u t} \geq\|A\|_{\infty \rightarrow 1} / 4$.

Mostremos o outro lado da desigualdade. Sejam dois conjuntos quaisquer $S \subseteq[m]$ e $T \subseteq[n]$ e defina, para cada $i \in[m]$ e $j \in[n]$,

$$
\varepsilon_{i}=\left\{\begin{array}{ll}
1, & \text { se } i \in S ; \\
-1, & \text { se } i \notin S,
\end{array} \text { e } \delta_{j}= \begin{cases}1, & \text { se } j \in T \\
-1, & \text { se } j \notin T\end{cases}\right.
$$

Usando o fato que a soma de cada linha e de cada coluna de $A$ é igual a zero, obtemos

$$
\sum_{\substack{i \in S \\ j \in T}} a_{i j}=\sum_{i=1}^{m} \sum_{j=1}^{n} a_{i j} \frac{1+\varepsilon_{i}}{2} \cdot \frac{1+\delta_{j}}{2}=\frac{1}{4} \sum_{i=1}^{m} \sum_{j=1}^{n} a_{i j} \varepsilon_{i} \delta_{j} \leq \frac{1}{4}\|A\|_{\infty \rightarrow 1},
$$

como queríamos.

Dada uma matriz $A$ do tipo $m \times n$, definimos a matriz $B=\left(b_{i j}\right)$ do tipo $(m+1) \times(n+1)$ como

$$
B=\left(\begin{array}{ccccc}
a_{11} & a_{12} & \cdots & a_{1 n} & -\sum_{j=1}^{n} a_{1 j} \\
a_{21} & a_{22} & \cdots & a_{2 n} & -\sum_{j=1}^{n} a_{2 j} \\
\vdots & \vdots & \ddots & \vdots & \vdots \\
a_{m 1} & a_{m 2} & \cdots & a_{m n} & -\sum_{j=1}^{n} a_{m j} \\
-\sum_{i=1}^{m} a_{i 1} & -\sum_{i=1}^{m} a_{i 2} & \cdots & -\sum_{i=1}^{m} a_{i n} & \sum_{i=1}^{m} \sum_{j=1}^{n} a_{i j}
\end{array}\right)
$$


Note que a soma de cada linha e de cada coluna da matriz $B$ é igual a zero. Logo ela satisfaz o Lema 52. Porém a matriz $B$ possui uma propriedade a mais, que será mostrada no lema a seguir.

Lema 53. Seja A uma matriz do tipo $m \times n$ e considere a matriz $B$ definida em (4.9). Temos $\|A\|_{\text {cut }}=\|B\|_{\text {cut }}$.

Demonstração. Sejam $S \subseteq[m+1]$ e $T \subseteq[n+1]$, e definimos $S^{*} \subseteq[m]$ e $T^{*} \subseteq[n]$ como

$$
S^{*}=\left\{\begin{array}{ll}
S, & \text { se } m+1 \notin S ; \\
{[m] \backslash S,} & \text { se } m+1 \in S .
\end{array} \text { e } T^{*}= \begin{cases}T, & \text { se } n+1 \notin T ; \\
{[n] \backslash T,} & \text { se } n+1 \in T .\end{cases}\right.
$$

Mostremos que

$$
\left|\sum_{\substack{i \in S \\ j \in T}} b_{i j}\right|=\left|\sum_{\substack{i \in S^{*} \\ j \in T^{*}}} a_{i j}\right|
$$

para todo $S \subseteq[m+1]$ e $T \subseteq[n+1]$. Temos os seguintes casos:

Caso 1. Se $m+1 \notin S$ e $n+1 \notin T$, então $S^{*}=S$ e $T^{*}=T$. Portanto $b_{i j}=a_{i j}$ para todo $i \in S$ e $j \in T$, satisfazendo (4.10).

Caso 2. Se $m+1 \in S$ e $n+1 \notin T$, então

$$
\begin{aligned}
\left|\sum_{\substack{i \in S \\
j \in T}} b_{i j}\right| & =\left|\sum_{\substack{i \in S \backslash\{m+1\} \\
j \in T}} b_{i j}+\sum_{j \in T} b_{(m+1) j}\right|=\left|\sum_{\substack{i \in S \backslash\{m+1\} \\
j \in T}} a_{i j}+\sum_{j \in T}\left(-\sum_{i=1}^{m} a_{i j}\right)\right| \\
& =\left|\sum_{\substack{i \in S \backslash\{m+1\} \\
j \in T}} a_{i j}-\sum_{\substack{i \in[m] \\
j \in T}} a_{i j}\right|=\left|\sum_{\substack{i \in[m] \backslash S \\
j \in T}} a_{i j}\right| .
\end{aligned}
$$

Note que o caso $m+1 \notin S$ e $n+1 \in T$ é análogo.

Caso 3. Se $m+1 \in S$ e $n+1 \in T$, então

$$
\begin{aligned}
& \left|\sum_{\substack{i \in S \\
j \in T}} b_{i j}\right|=\left|\sum_{\substack{i \in S \backslash\{m+1\} \\
j \in T \backslash\{n+1\}}} b_{i j}+\sum_{i \in S \backslash\{m+1\}} b_{i(n+1)}+\sum_{j \in T \backslash\{n+1\}} b_{(m+1) j}+b_{(m+1)(n+1)}\right| \\
& =\left|\sum_{\substack{i \in S \backslash\{m+1\} \\
j \in T \backslash\{n+1\}}} a_{i j}-\sum_{i \in S \backslash\{m+1\}} \sum_{j=1}^{n} a_{i j}-\sum_{j \in T \backslash\{n+1\}} \sum_{i=1}^{m} a_{i j}+\sum_{i=1}^{m} \sum_{j=1}^{n} a_{i j}\right| \\
& =\left|\sum_{\substack{i \in S \backslash\{m+1\} \\
j \in T \backslash\{n+1\}}} a_{i j}-\sum_{\substack{i \in S \backslash\{m+1\} \\
j \in[n]}} a_{i j}-\sum_{\substack{i \in[m] \\
j \in T \backslash\{n+1\}}} a_{i j}+\sum_{\substack{i \in[m] \\
j \in[n]}} a_{i j}\right| \\
& =\left|\sum_{\substack{i \in S \backslash\{m+1\} \\
j \in[n] \backslash T}} a_{i j}+\sum_{\substack{i \in[m] \\
j \in[n] \backslash T}} a_{i j}\right| \\
& =\left|\sum_{\substack{i \in[m] \backslash S \\
j \in[n] \backslash T}} a_{i j}\right| .
\end{aligned}
$$


Portanto

$$
\|B\|_{c u t}=\max _{\substack{S \subseteq[m+1] \\ T \subseteq[n+1]}}\left|\sum_{\substack{i \in S \\ j \in T}} b_{i j}\right|=\max _{\substack{S \subseteq[m+1] \\ T \subseteq[n+1]}}\left|\sum_{\substack{i \in S^{*} \\ j \in T^{*}}} a_{i j}\right|=\max _{\substack{X \subseteq[m] \\ Y \subseteq[n]}}\left|\sum_{\substack{i \in X \\ j \in Y}} a_{i j}\right|=\|A\|_{c u t},
$$

como queríamos.

Vamos mostrar uma relação entre a norma do corte e a Desigualdade de Grothendieck.

Proposição 54. Existe um algoritmo em tempo polinomial que produz, para toda matriz real $A$ do tipo $m \times n$, um real $\alpha>0$ tal que

$$
\|A\|_{c u t} \leq \alpha \leq K_{G}\|A\|_{c u t} .
$$

Demonstração. Fixe uma matriz $A$ do tipo $m \times n$ qualquer e considere a matriz $B$ definida em (4.9). Pela Desigualdade de Grothendieck (46),

$$
\|B\|_{\infty \rightarrow 1} \leq \operatorname{SDP}(B) \leq K_{G}\|B\|_{\infty \rightarrow 1} .
$$

Dividindo tudo por quatro e aplicando o Lema 52, obtemos

$$
\|A\|_{c u t} \leq \frac{\operatorname{SDP}(B)}{4} \leq K_{G}\|A\|_{c u t}
$$

como queríamos.

A seguir, apresentaremos o Teorema de Alon e Naor, que é o teorema principal do nosso estudo.

Teorema 55 (Alon-Naor, 2006). Existe um algoritmo em tempo polinomial que produz, para toda matriz real $A$ do tipo $m \times n$, dois subconjuntos $S_{0} \subseteq[m]$ e $T_{0} \subseteq[n]$ tais que

$$
\left|\sum_{\substack{i \in S_{0} \\ j \in T_{0}}} a_{i j}\right| \geq K_{G}^{-1}\|A\|_{c u t}
$$

Demonstração. Fixe $A$ uma matriz do tipo $m \times n$ e considere a matriz $B$ definida em (4.9). Sejam $\left\{u_{i}\right\}_{i=1}^{m+1}$ e $\left\{v_{j}\right\}_{j=1}^{n+1}$ vetores unitários em $\mathbb{R}^{(m+1)+(n+1)}$ que atingem o máximo de $\sum_{i j} a_{i j}\left\langle u_{i}, v_{j}\right\rangle$.

Pelo Lema 50 e pela Proposição 54, existem vetores unitários $\left\{u_{i}^{\prime}\right\}_{i=1}^{m+1}$ e $\left\{v_{j}^{\prime}\right\}_{j=1}^{n+1}$ em $\mathbb{R}^{(m+1)+(n+1)}$ tais que

$$
\begin{aligned}
\mathbb{E}\left[\sum_{\substack{i \in[m+1] \\
j \in[n+1]}} b_{i j} \operatorname{sign}\left\langle u_{i}^{\prime}, r\right\rangle \operatorname{sign}\left\langle v_{j}^{\prime}, r\right\rangle\right] & =\frac{2}{\pi} c \sum_{\substack{i \in[m+1] \\
j \in[n+1]}} b_{i j}\left\langle u_{i}, v_{j}\right\rangle \\
& =\frac{2}{\pi} c \operatorname{SDP}(B) \\
& \geq 4 K_{G}^{-1}\|A\|_{\text {cut }} .
\end{aligned}
$$

Escolha um vetor aleatório $\tilde{r}$ uniformemente ao acaso sobre a esfera unitária tal que

$$
\sum_{\substack{i \in[m+1] \\ j \in[n+1]}} b_{i j} \operatorname{sign}\left\langle u_{i}^{\prime}, \tilde{r}\right\rangle \operatorname{sign}\left\langle v_{j}^{\prime}, \tilde{r}\right\rangle \geq \mathbb{E}\left[\sum_{\substack{i \in[m+1] \\ j \in[n+1]}} b_{i j} \operatorname{sign}\left\langle u_{i}^{\prime}, r\right\rangle \operatorname{sign}\left\langle v_{j}^{\prime}, r\right\rangle\right] .
$$


$\operatorname{Sejam} \varepsilon_{i}=\operatorname{sign}\left\langle u_{i}^{\prime}, \tilde{r}\right\rangle$ e $\delta_{j}=\operatorname{sign}\left\langle v_{j}^{\prime}, \tilde{r}\right\rangle$ e considere os conjuntos

$$
S=\left\{i \in[m+1]: \varepsilon_{i}=1\right\} \text { e } T=\left\{j \in[n+1]: \delta_{j}=1\right\} .
$$

Temos

$$
\sum_{\substack{i \in S \\ j \in T}} b_{i j}=\sum_{\substack{i \in[m+1] \\ j \in[n+1]}} b_{i j} \frac{\varepsilon_{i}+1}{2} \cdot \frac{\delta_{j}+1}{2}=\frac{1}{4} \sum_{\substack{i \in[m+1] \\ j \in[n+1]}} b_{i j} \varepsilon_{i} \delta_{j} .
$$

Logo, pela desigualdade (4.11),

$$
\left|\sum_{\substack{i \in S \\ j \in T}} b_{i j}\right| \geq K_{G}^{-1}\|A\|_{c u t} .
$$

Pelo argumento do Lema 53, tomando $S^{*}=S_{0}$ e $T^{*}=T_{0}$, obtemos

$$
\left|\sum_{\substack{i \in S_{0} \\ j \in T_{0}}} a_{i j}\right|=\left|\sum_{\substack{i \in S \\ j \in T}} b_{i j}\right| \geq K_{G}^{-1}\|A\|_{c u t},
$$

como queríamos. 


\section{Capítulo 5}

\section{Aplicações da Norma de Corte}

Alon e Naor, em [AN06], obtiveram um algoritmo de aproximação em tempo polinomial que produz, para toda matriz $A=\left(a_{i j}\right)$ do tipo $m \times n$, dois subconjuntos $S_{0} \subseteq[m]$ e $T_{0} \subseteq[n]$ tais que

$$
\left|\sum_{\substack{i \in S_{0} \\ j \in T_{0}}} a_{i j}\right| \geq K_{G}^{-1}\|A\|_{c u t} .
$$

A prova pode ser vista no Capítulo 4, Teorema 55.

O objetivo deste capítulo é apresentar aplicações o Teorema 55. Todas as aplicações expostas aqui são devidas a Alon e Naor em [AN06].

Serão três aplicações: corte de grafo; Lema da Regularidade de Szemerédi; e Teorema da Decomposição Matricial de Frieze e Kannan.

\subsection{Relação entre norma de corte e corte de grafo}

Seja $G_{W}=(V, E)$ um grafo com pesos qualquer com $|V|=n$ e $|E|=m$ e com matriz de pesos $W$. Seja $D$ um grafo orientado qualquer de $G$. Definimos a matriz $A\left(D, e_{1}, \ldots, e_{m}\right)=\left(a_{i j}\right)$ do tipo $2 m \times n$ satisfazendo as seguintes condições: (1) Se a aresta $e_{i}$ está orientada de $v_{j}$ para $v_{k}$, então

$$
\left\{\begin{array}{l}
a_{2 i-1, j}=a_{2 i, k}=w_{j k} ; \\
a_{2 i-1, k}=a_{2 i, j}=-w_{j k} .
\end{array}\right.
$$

(2) As entradas restantes de $A\left(D, e_{1}, \ldots, e_{m}\right)$ são iguais a zero.

Exemplo 56. Seja $G=C_{4}$ o circuito de ordem quatro com vértices $V=\left\{v_{1}, v_{2}, v_{3}, v_{4}\right\}$ com pesos $w\left(v_{1} v_{2}\right)=1, w\left(v_{2} v_{3}\right)=5, w\left(v_{3} v_{4}\right)=2$ e $w\left(v_{1} v_{4}\right)=10$, e oriente as arestas da forma $v_{1} \vec{v}_{2}, v_{2} \vec{v}_{3}, v_{4} \vec{v}_{3}$ e $v_{4} \vec{v}_{1}$, seja $\vec{C}_{4}$ o grafo orientado obtido.

Vamos encontrar a matriz $A\left(\vec{C}_{4}, v_{1} \vec{v}_{2}, v_{2} \vec{v}_{3}, v_{4} \vec{v}_{3}, v_{4} \vec{v}_{1}\right)$.

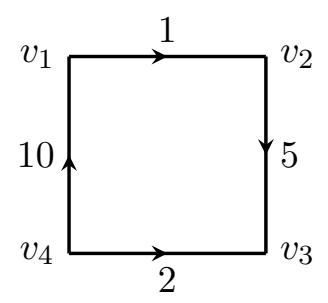

Figura 5.1: Um grafo orientado $\vec{C}_{4}$ 
Para cada aresta $v_{1} \vec{v}_{2}, v_{2} \vec{v}_{3}, v_{4} \vec{v}_{3}$ e $v_{4} \vec{v}_{1}$ obtemos, respectivamente, as seguintes equações.
(1) $\left\{\begin{array}{l}a_{11}=a_{22}=1 \\ a_{12}=a_{21}=-1\end{array}\right.$
(3) $\left\{\begin{array}{l}a_{54}=a_{63}=2 \\ a_{53}=a_{64}=-2 .\end{array}\right.$
(2) $\left\{\begin{array}{l}a_{32}=a_{43}=5 \\ a_{33}=a_{42}=-5 .\end{array}\right.$
(4) $\left\{\begin{array}{l}a_{74}=a_{81}=10 \\ a_{71}=a_{84}=-10\end{array}\right.$

A matriz $A\left(\vec{C}_{4}, v_{1} \vec{v}_{2}, v_{2} \vec{v}_{3}, v_{4} \vec{v}_{3}, v_{4} \vec{v}_{1}\right)$ é igual a

$$
A\left(\vec{C}_{4}, v_{1} \vec{v}_{2}, v_{2} \vec{v}_{3}, v_{4} \vec{v}_{3}, v_{4} \vec{v}_{1}\right)=\left[\begin{array}{cccc}
1 & -1 & 0 & 0 \\
-1 & 1 & 0 & 0 \\
0 & 5 & -5 & 0 \\
0 & -5 & 5 & 0 \\
0 & 0 & -2 & 2 \\
0 & 0 & 2 & -2 \\
-10 & 0 & 0 & 10 \\
10 & 0 & 0 & -10
\end{array}\right] .
$$

Proposição 57. Para todo grafo com pesos $G_{W}=(V, E),|V|=n$ e $|E|=m$ existe uma matriz $A$ do tipo $2 m \times n$ tal que

$$
\operatorname{MAXCUT}(W)=\|A\|_{c u t} .
$$

Demonstração. Mostremos para grafos sem pesos, o caso com pesos é análogo. Seja $G=(V, E)$ um grafo qualquer e oriente as arestas arbitrariamente, formando um grafo orientado $D$. Sejam $V=$ $\left\{v_{1}, \ldots, v_{n}\right\}$ e $A=A\left(D, e_{1}, \ldots, e_{m}\right)=\left(a_{i j}\right)$ a matriz do tipo $2 m \times n$ definida acima. Mostremos que $\operatorname{MAXCUT}(G)=\|A\|_{\text {cut }}$.

Fixe $T \subseteq[n]$ qualquer. Seja $S \subseteq[2 m]$ satisfazendo a seguinte condição: Se existe $j \in T$ tal que $a_{i j}=1$, então $i \in S$. Caso contrário, $i \notin S(\star)$.

Tomando $X=\left\{v_{j}: j \in T\right\}$, vale que

$$
\sum_{\substack{i \in S \\ j \in T}} a_{i j}=e\left(X, X^{c}\right)
$$

Logo, existem $S^{\prime} \subseteq[2 m]$ e $T^{\prime} \subseteq[n]$ tais que

$$
\operatorname{MAXCUT}(G)=\max \left\{e\left(X, X^{c}\right): X \subseteq V\right\}=\sum_{\substack{i \in S^{\prime} \\ j \in T^{\prime}}} a_{i j} \leq\|A\|_{c u t} .
$$

Seja $A^{\prime}=\left(a_{i j}\right)_{i \in S^{\prime}, j \in T^{\prime}}$. Mostremos que para todo $I \subseteq[2 m]$ e $J \subseteq[n]$, vale que

$$
\sum_{\substack{i \in I \\ j \in J}} a_{i j} \leq \sum_{\substack{i \in S^{\prime} \\ j \in T^{\prime}}} a_{i j}
$$

Claramente a desigualdade (5.1) implica que $\|A\|_{\text {cut }} \leq \operatorname{MAXCUT}(G)$. 
Sejam $I \subseteq[2 m]$ e $J \subseteq[n]$ conjuntos quaisquer e considere $C=\left(a_{i j}\right)_{i \in I, j \in J}$ uma submatriz de $A$. Se $I$ satisfaz ( $\star$, então a desigualdade (5.1) é satisfeita. Suponhamos que $I$ não satisfaz ( $\star$ ). Temos dois casos (não-exclusivos):

1. Existem $k \notin I$ e $l \in J$ tais que $a_{k l}=1$; e

2. Existe $k \in I$ tal que para todo $l \in J$, temos $a_{k l}=-1$ ou $a_{k l}=0$.

Sejam

$$
\begin{aligned}
& I_{1}=\left\{k \notin I: \text { existe } l \in J \text { tal que } a_{k l}=1\right\}, \\
& I_{2}=\left\{k \in I: \text { para todo } l \in J, a_{k l}=-1 \text { ou } a_{k l}=0\right\} .
\end{aligned}
$$

Assim, o conjunto $\left(I \backslash I_{2}\right) \cup I_{1} \subseteq[2 m]$ satisfaz a condição $(\star)$. De fato, para todo $i \in\left(I \backslash I_{2}\right) \cup I_{1}$ existe $j \in J$ tal que $a_{i j}=1$, e para todo $i \notin\left(I \backslash I_{2}\right) \cup I_{1}$, temos $i \in\left(\left(I \backslash I_{2}\right) \cup I_{1}\right)^{c}=\left(I \cap I_{1}\right)^{c} \cup\left(I_{2} \cap I_{1}^{c}\right)$, logo para todo $j \in J$ temos $a_{i j}=-1$ ou $a_{i j}=0$. Tomando $X=\left\{v_{j}: j \in J\right\}$, temos

$$
\sum_{\substack{i \in\left(I \backslash I_{2}\right) \cup I_{1} \\ j \in J}} a_{i j}=e\left(X, X^{c}\right) .
$$

Como $I_{1}$ contribui com termos não-negativos para a soma, e $I_{2}$ contribui com termos não-positivos para a soma, temos

$$
\sum_{\substack{i \in I \\ j \in J}} a_{i j} \leq \sum_{\substack{i \in\left(I \backslash I_{2}\right) \cup I_{1} \\ j \in J}} a_{i j} .
$$

Portanto a desigualdade (5.1) vale para todo $I \subseteq[2 m]$ e $J \subseteq[n]$.

A partir do Teorema 55 e do Teorema 57, obtemos um algoritmo de $\rho$-aproximação para o corte máximo de um grafo, apesar de a garantia de desempenho não é tão boa quanto a garantia de desempenho $\rho=0.87856$ de Goemans e Williamson [GW95].

Corolário 58. Seja $G=(V, E)$ um grafo com $|V|=n$ e $|E|=m$. Existe um algoritmo em tempo polinomial que produz uma matriz $A$ do tipo $2 m \times n$ e subconjuntos $S_{0} \subseteq[2 m]$ e $T_{0} \subset[n]$ tais que

$$
\left|\sum_{\substack{i \in S_{0} \\ j \in T_{0}}} a_{i j}\right| \geq K_{G}^{-1} \operatorname{MAXCUT}(G) .
$$

Demonstração. Fixe um grafo $G=(V, E)$ com $|V|=n$ e $|E|=m$ qualquer. Pela Proposição 57, existe uma matriz $A$ do tipo $2 m \times n$ tal que

$$
\operatorname{MAXCUT}(G)=\|A\|_{c u t} .
$$

Pelo Teorema 55, existe um algoritmo em tempo polinomial que produz $S_{0} \subseteq[2 m]$ e $T_{0} \subseteq[n]$ tais que

$$
\|A\|_{c u t} \leq K_{G}\left|\sum_{\substack{i \in S_{0} \\ j \in T_{0}}} a_{i j}\right| .
$$

Portanto

$$
\operatorname{MAXCUT}(G) \leq K_{G}\left|\sum_{\substack{i \in S_{0} \\ j \in T_{0}}} a_{i j}\right|,
$$

como queríamos. 
Sabendo que o Problema do Corte Máximo é SNP-difícil, temos o seguinte corolário da Proposição 57 .

Corolário 59. O Problema da Norma de Corte é SNP-difícil.

Ademais, pelo Lema 52, concluímos também que determinar o valor exato da norma $\|\cdot\|_{\infty \rightarrow 1}$ de uma matriz é SNP-difícil.

\subsection{Lema da Regularidade de Szemerédi}

O Lema da Regularidade de Szemerédi é um lema fundamental na teoria dos grafos. Foi primeiramente utilizado na prova da Conjectura de Erdôs e Turán [ET36] sobre progressões aritméticas em subconjuntos suficientemente densos de inteiros. Nesta seção apresentaremos o Lema da Regularidade de Szemerédi e a sua versão algorítmica. Recomendamos uma resenha de Komlós e Simonovits [KS96] sobre o lema.

Sejam $G=(V, E)$ um grafo e $X, Y \subseteq V$ subconjuntos disjuntos dos vértices de $G$. Denotemos por

$$
\mathrm{d}(X, Y)=\frac{e(X, Y)}{|X||Y|}
$$

a densidade do par $(X, Y)$. Note que, para todo par $(X, Y)$, vale $0 \leq \mathrm{d}(X, Y) \leq 1$. Dados $\varepsilon>0$ e $A, B \subseteq V$ dois subconjuntos disjuntos, o par $(A, B)$ é dito $\varepsilon$-regular se para todo $X \subseteq A$ e $Y \subseteq B$ tais que

$$
|X| \geq \varepsilon|A| \text { e }|Y| \geq \varepsilon|B|
$$

então

$$
|\mathrm{d}(X, Y)-\mathrm{d}(A, B)| \leq \varepsilon .
$$

Uma partição $\left\{V_{0}, V_{1}, \ldots, V_{k}\right\}$ de $V$ é dita $\varepsilon$-regular de $G$ se satisfaz as seguintes condições:

1. $\left|V_{0}\right| \leq \varepsilon|V|$;

2. $\left|V_{1}\right|=\ldots=\left|V_{k}\right|$;

3. todos os pares $\left(V_{i}, V_{j}\right)$ com $i \neq j$ são $\varepsilon$-regulares, a menos de no máximo $\varepsilon k^{2}$ de tais pares.

Dizemos que uma partição é $\varepsilon$-irregular se não é $\varepsilon$-regular.

O Lema da Regularidade de Szemerédi pode ser enunciado como segue.

Teorema 60 (Szemerédi, 1978). Para todo $\varepsilon>0$ e para todo inteiro $m>0$ existe um inteiro $M>0$ tal que todo grafo de ordem pelo menos $m$ admite uma partição $\varepsilon$-regular $\left\{V_{0}, V_{1}, \ldots, V_{k}\right\}$ para algum $m \leq k \leq M$.

Alon, Duke, Lefmann, Rödl e Yuster [ADL $\left.{ }^{+} 94\right]$ provaram a seguinte versão algorítmica do Teorema 60. Denotemos por $M(n)$ o tempo necessário para que um algoritmo multiplique duas matrizes quadradas com entradas em $\{0,1\}$.

Teorema 61 (Alon-Duke-Lefmann-Rödl-Yuster, 1994). Existe um algoritmo determinístico $\mathcal{A}_{0}$ que, dado $\varepsilon>0, k_{0} \geq 1$, e um grafo $G$, produz uma partição $\varepsilon$-regular para $G$ em $k$ partes para algum $k$ satisfazendo $k_{0} \leq k \leq K_{0}^{\prime}$, onde $K_{0}^{\prime}=K_{0}^{\prime}\left(\varepsilon, k_{0}\right)$ depende somente de $\varepsilon$ e $k_{0}$. Ademais, o algoritmo $\mathcal{A}_{0}$ executa em tempo $O(M(n))=O\left(n^{2.376}\right)$ se $G$ tem $n$ vértices.

Considere agora o seguinte problema de decisão.

Problema 62. Dado um grafo $G$, um par de subconjuntos de vértices $(U, W)$ em $G$ não-vazios e disjuntos, e um positivo $\varepsilon$, decidir se $(U, W)$ é $\varepsilon$-regular com respeito a $G$.

O problema acima é coNP-completo, e a prova pode ser vista em [ADL ${ }^{+}$94]. Porém, como se pode ver em [ADL ${ }^{+}$94], para provar o Teorema 61, é suficiente resolver uma versão aproximada do problema de decisão acima. Por exemplo, o seguinte resultado [DLR95] é suficiente. 
Teorema 63 (Duke-Lefmann-Rödl, 1995). Existe um algoritmo $\mathcal{A}_{1}$ para o qual a seguinte afirmação é válida. Quando $\mathcal{A}_{1}$ recebe como entrada um $\varepsilon>0$ e um grafo bipartido $G=(A, B ; E)$ com $|A|=|B|=m \geq(2 / \varepsilon)^{5}$, o algoritmo ou afirma que $G$ é $\varepsilon$-regular, ou produz uma testemunha para a $\varepsilon^{\prime}$-irregularidade de $G$, onde $\varepsilon^{\prime}=\varepsilon_{\mathcal{A}_{1}}^{\prime}(\varepsilon)=\varepsilon^{5} / 16$. O tempo de execução de $\mathcal{A}_{1}$ é $O(M(n))=O\left(n^{2,376}\right)$.

Kohayakawa, Rödl e Thoma [KRT03] encontraram um outro algoritmo:

Teorema 64 (Kohayakawa, Rödl e Thoma, 2003). Existe um algoritmo $\mathcal{A}$ para o qual a seguinte afirmação é válida. Quando $\mathcal{A}$ recebe como entrada um $\varepsilon>0$ e um grafo bipartido $G=(A, B ; E)$ com $|A|=|B|=m \geq m_{0}(\varepsilon)$, o algoritmo afirma ou que $G$ é $\varepsilon$-regular, ou produz uma testemunha para a $\varepsilon^{\prime}$-irregularidade de $G$, onde $\varepsilon^{\prime}=\varepsilon_{\mathcal{A}}^{\prime}(\varepsilon)=\varepsilon^{20} / 10^{24}$. O tempo de execução de $\mathcal{A}$ é $O\left(m^{2}\right)$.

A partir desse Teorema, em [KRT03] obtiveram um algoritmo para o Lema da Regularidade.

Corolário 65. Existe um algoritmo determinístico $\mathcal{A}_{0}^{\prime}$ que, dado $\varepsilon>0, k_{0} \geq 1$, e um grafo $G$, produz uma partição $\varepsilon$-regular para $G$ em $k$ partes para algum $k$ satisfazendo $k_{0} \leq k \leq K_{0}^{\prime \prime}$, onde $K_{0}^{\prime \prime}=$ $K_{0}^{\prime \prime}\left(\varepsilon, k_{0}\right)$ depende somente de $\varepsilon$ e $k_{0}$. Ademais, o algoritmo $\mathcal{A}_{0}^{\prime}$ executa em tempo $O\left(n^{2}\right)$ se $G$ tem $n$ vértices.

Usando o Teorema 55, Alon e Naor [AN06] obtiveram um algoritmo que, dado um grafo $G=$ $(V, E)$, então para todo par de subconjuntos disjuntos de vértices $\varepsilon$-irregular, produz construtivamente um par de subconjuntos disjuntos de vértices $\varepsilon^{3} / 2$-irregular.

Lema 66. Sejam $G=(V, E)$ um grafo e $X, Y \subseteq V$ subconjuntos disjuntos de $V$ com $|X|=|Y|=n$. Se o par $(X, Y)$ é $\varepsilon$-irregular, então existe um algoritmo em tempo polinomial que produz dois conjuntos $S \subseteq X$ e $T \subseteq Y$ tais que

$$
|S| \geq \frac{1}{2} \varepsilon^{3} n,|T| \geq \frac{1}{2} \varepsilon^{3} n e|\mathrm{~d}(S, T)-\mathrm{d}(X, Y)| \geq \frac{1}{2} \varepsilon^{3} .
$$

Demonstração. Sejam $G=(V, E)$ um grafo, e $X, Y \subseteq V$ subconjuntos disjuntos. Considere a matriz $A=\left(a_{x y}\right)_{(x, y) \in X \times Y}$ dada por

$$
a_{x y}=\left\{\begin{array}{l}
1-\mathrm{d}(X, Y), \text { se }\{x, y\} \in E, \\
-\mathrm{d}(X, Y), \text { se }\{x, y\} \notin E .
\end{array}\right.
$$

Pela definição da matriz $A$, se $S \subseteq X$ e $T \subseteq Y$, então

$$
\begin{aligned}
\sum_{(x, y) \in S \times T} a_{x y} & =\sum_{\substack{x y \in E \\
(x, y) \in S \times T}}(1-\mathrm{d}(X, Y))+\sum_{\substack{x y \notin E \\
(x, y) \in S \times T}}(-\mathrm{d}(X, Y)) \\
& =e(S, T)-\sum_{(x, y) \in S \times T} \mathrm{~d}(X, Y) \\
& =e(S, T)-|S||T| \mathrm{d}(X, Y) \\
& =|S||T|(\mathrm{d}(S, T)-\mathrm{d}(X, Y)) .
\end{aligned}
$$

Logo,

$$
\left|\sum_{(x, y) \in S \times T} a_{x y}\right|=|S| \cdot|T| \cdot|\mathrm{d}(S, T)-\mathrm{d}(X, Y)| .
$$

Como o par $(X, Y)$ é $\varepsilon$-irregular, existem $S \subseteq X$ e $T \subseteq Y$ tais que $|S| \geq \varepsilon n,|T| \geq \varepsilon n$ e $\mid \mathrm{d}(S, T)-$ $\mathrm{d}(X, Y) \mid \geq \varepsilon$. Juntamente com a equação (5.3),

$$
\left|\sum_{(x, y) \in S \times T} a_{x y}\right| \geq \varepsilon^{3} n^{2} .
$$


Logo

$$
\|A\|_{c u t} \geq \varepsilon^{3} n^{2} .
$$

Mostremos que, para todo $S \subseteq X$ e $T \subseteq Y$,

$$
\min \left\{n|S|, n|T|, n^{2}|\mathrm{~d}(S, T)-\mathrm{d}(X, Y)|\right\} \geq\left|\sum_{(x, y) \in S \times T} a_{x y}\right| .
$$

Como $|S| \leq n$ e $|T| \leq n$, vale

$$
n^{2}|\mathrm{~d}(S, T)-\mathrm{d}(X, Y)| \geq|S| \cdot|T| \cdot|\mathrm{d}(S, T)-\mathrm{d}(X, Y)|=\left|\sum_{(x, y) \in S \times T} a_{x y}\right| .
$$

Como $\left|a_{x y}\right| \leq 1$ para todo $(x, y) \in X \times Y$, pela desigualdade triangular,

$$
\left|\sum_{(x, y) \in S \times T} a_{x y}\right| \leq \sum_{(x, y) \in S \times T}\left|a_{x y}\right| \leq|S| \cdot|T| .
$$

Portanto,

$$
n|S| \geq\left|\sum_{(x, y) \in S \times T} a_{x y}\right| \text { e } n|T| \geq\left|\sum_{(x, y) \in S \times T} a_{x y}\right|,
$$

provando a desigualdade (5.5).

Pelo Teorema 55, podemos encontrar, em tempo polinomial, dois subconjuntos $S_{0} \subseteq X$ e $T_{0} \subseteq Y$ tais que

$$
\left|\sum_{(x, y) \in S_{0} \times T_{0}} a_{x y}\right| \geq K_{G}^{-1}\|A\|_{\text {cut }} .
$$

Assim, pelas desigualdades (5.4), (5.5) e (5.6), e usando que $K_{G} \leq 2$, obtemos

$$
\min \left\{n\left|S_{0}\right|, n\left|T_{0}\right|, n^{2}\left|\mathrm{~d}\left(S_{0}, T_{0}\right)-\mathrm{d}(X, Y)\right|\right\} \geq \frac{1}{2} \varepsilon^{3} n^{2},
$$

como queríamos.

\subsection{Decomposição Matricial de Frieze e Kannan}

Uma matriz $D=\left(d_{i j}\right)$ do tipo $m \times n$ é dita matriz de corte se existem dois subconjuntos $S \subseteq[m]$ e $T \subseteq[n]$ e um real $d$ tais que para todo $(i, j) \in[m] \times[n]$,

$$
d_{i j}=\left\{\begin{array}{l}
d \text { se }(i, j) \in S \times T \\
0 \text { se }(i, j) \notin S \times T
\end{array}\right.
$$

Denotemos a matriz $D$ por $C U T(S, T, d)$. Frieze e Kannan [FK99] mostraram que para todo $\varepsilon>0$ existe um inteiro $s=O\left(1 / \varepsilon^{2}\right)$ tal que para toda matriz $A$ do tipo $m \times n$ com cada entrada menor ou igual a $1 \mathrm{em}$ valor absoluto, existem matrizes de corte $D_{1}, \ldots, D_{s}$ tais que

$$
\left\|A-\sum_{k=1}^{s} D_{k}\right\|_{c u t} \leq \varepsilon m n .
$$

Ademais, as matrizes de corte $D_{1}, \ldots, D_{s}$ podem ser encontradas em tempo $C(\varepsilon)(m n)^{O(1)}$. 
Frieze e Kannan, em [FK96], aplicaram o algoritmo de decomposição matricial em dois ramos de pesquisa. A primeira aplicação foi obter uma versão mais fraca do Lema da Regularidade (veja [FK96], [FK96] e [DKM ${ }^{+}$12] para mais detalhes), e a segunda foi estudar alguns problemas de grafos, principalmente o Problema do Corte Máximo com Peso.

Alon e Naor [AN06] obtiveram o Teorema de Frieze e Kannan a partir do Teorema 55.

Teorema 67 (Frieze-Kannan, 1996). Para todo $\varepsilon>0$ existe um inteiro $s=O\left(1 / \varepsilon^{2}\right)$ tal que para toda matriz $A=\left(a_{i j}\right)$ do tipo $m \times n$ tal que $\left|a_{i j}\right| \leq 1$ para todo $i, j$, existem matrizes de corte $D_{1}, \ldots, D_{s}$ satisfazendo

$$
\left\|A-\sum_{k=1}^{s} D_{k}\right\|_{c u t} \leq \varepsilon m n .
$$

Essas matrizes de corte $D_{1}, \ldots, D_{\text {s }}$ podem ser encontradas em tempo $C(\varepsilon)(m n)^{O(1)}$.

Demonstração. Denotemos $A_{0}=A$ e assumimos que as matrizes de corte $D_{1}, \ldots, D_{r}$ já estão definidas. Definimos

$$
A_{r}=\left(a_{i j}(r)\right)=A-\sum_{k=1}^{r} D_{k} .
$$

Queremos $r$ tal que $\left\|A_{r}\right\|_{\text {cut }} \leq \varepsilon m n$. Assumimos que $\left\|A_{r}\right\|_{c u t}>\varepsilon m n$. Pelo Teorema 55, podemos encontrar, em tempo polinomial, dois subconjuntos $S \subseteq[m]$ e $T \subseteq[n]$ tais que

$$
\left|\sum_{\substack{i \in S \\ j \in T}} a_{i j}(r)\right| \geq K_{G}^{-1}\left\|A_{r}\right\|_{c u t}>K_{G}^{-1} \varepsilon m n .
$$

Seja

$$
d=\frac{1}{|S||T|} \sum_{\substack{i \in S \\ j \in T}} a_{i j}(r) .
$$

Definimos $D_{r+1}=C U T(S, T, d)$. Por definição, $A_{r+1}=\left(a_{i j}(r+1)\right)=A_{r}-D_{r+1}$. Assim

$$
a_{i j}(r+1)=\left\{\begin{array}{l}
a_{i j}(r), \text { se } i \notin S \text { ou } j \notin T \\
a_{i j}(r)-d, \text { se } i \in S \text { e } j \in T
\end{array}\right.
$$

Somando todos os $a_{i j}(r+1)^{2}$, obtemos

$$
\begin{aligned}
\sum_{i j} a_{i j}(r+1)^{2} & =\sum_{\substack{i \in S \\
j \in T}} a_{i j}(r+1)^{2}+\sum_{\substack{i \notin S \\
j \in T}} a_{i j}(r+1)^{2}+\sum_{\substack{i \in S \\
j \notin T}} a_{i j}(r+1)^{2} \\
& =\sum_{\substack{i \in S \\
j \in T}}\left(a_{i j}(r)-d\right)^{2}+\sum_{\substack{i \notin S \\
j \in T}} a_{i j}(r)^{2}+\sum_{\substack{i \in S \\
j \notin T}} a_{i j}(r)^{2} .
\end{aligned}
$$

Como

$$
\begin{aligned}
\sum_{\substack{i \in S \\
j \in T}}\left(a_{i j}(r)-d\right)^{2} & =\sum_{\substack{i \in S \\
j \in T}} a_{i j}(r)^{2}-2 d \sum_{\substack{i \in S \\
j \in T}} a_{i j}(r)+|S||T| d^{2} \\
& =\sum_{\substack{i \in S \\
j \in T}} a_{i j}(r)^{2}-\frac{1}{|S||T|}\left(\sum_{\substack{i \in S \\
j \in T}} a_{i j}(r)\right)^{2},
\end{aligned}
$$


e sabendo que

$$
\sum_{i j} a_{i j}(r)^{2}=\sum_{\substack{i \in S \\ j \in T}} a_{i j}(r)^{2}+\sum_{\substack{i \notin S \\ j \in T}} a_{i j}(r)^{2}+\sum_{\substack{i \in S \\ j \notin T}} a_{i j}(r)^{2},
$$

concluímos que a soma (5.9) é igual a

$$
\sum_{i j} a_{i j}(r+1)^{2}=\sum_{i j} a_{i j}(r)^{2}-\frac{1}{|S||T|}\left(\sum_{\substack{i \in S \\ i \in T}} a_{i j}(r)\right)^{2} .
$$

Aplicando a desigualdade (5.8) em (5.10), obtemos

$$
\sum_{i j} a_{i j}(r+1)^{2} \leq \sum_{i j} a_{i j}(r)^{2}-K_{G}^{-2} \varepsilon^{2} m n
$$

Aplicando a desigualdade (5.11) $r$ vezes,

$$
0 \leq \sum_{i j} a_{i j}(r)^{2} \leq \sum_{i j} a_{i j}^{2}-r K_{G}^{-2} \varepsilon^{2} m n \leq m n-r K_{G}^{-2} \varepsilon^{2} m n
$$

onde a última igualdade vale pois $\left|a_{i j}\right| \leq 1$ para todo $i \in[m]$ e $j \in[n]$. Logo o processo acima deve terminar após $r=\left\lceil K_{G}^{2} \varepsilon^{-2}\right\rceil=O\left(\varepsilon^{-2}\right)$ passos. Portanto, para esse $r$, temos

$$
\left\|A_{r}\right\|_{c u t} \leq \varepsilon m n
$$

como queríamos.

O passo principal da demonstração é produzir conjuntos $S, T$ em tempo polinomial satisfazendo a desigualdade (5.8). Note que obtemos uma aproximação multiplicativa. O algoritmo de Frieze e Kannan [FK99] garante uma aproximação aditiva, isto é, o algoritmo recebe uma matriz $A=\left(a_{i j}\right)$ do tipo $m \times n$ e $\varepsilon>0$ e produz, em tempo $2^{1 / \varepsilon^{O(1)}}(m n)^{O(1)}$, subconjuntos $S \subseteq[m]$ e $T \subseteq[n]$ satisfazendo

$$
\left|\sum_{\substack{i \in S \\ j \in T}} a_{i j}\right| \geq\|A\|_{c u t}-\varepsilon m n .
$$

Note que o tempo de execução do algoritmo em [FK99] não é polinomial se, digamos, $\varepsilon=n^{\Omega(1)}$. Logo o algoritmo de Frieze e Kannan é relevante somente para instâncias "densas". Ademais, o algoritmo que obtivemos no Teorema 67 vale para qualquer valor de $\|A\|_{c u t}$, o que permite tomar qualquer $\varepsilon>0$. 


\section{Referências Bibliográficas}

[AB09] S. Arora e B. Barak. Computational complexity. Cambridge University Press, Cambridge, 2009. A modern approach. 14, 16

[ABI86] N. Alon, L. Babai e A. Itai. A fast and simple randomized parallel algorithm for the maximal independent set problem. J. Algorithms, 7(4):567-583, 1986. 26

$\left[\mathrm{ADL}^{+} 94\right]$ N. Alon, R. A. Duke, H. Lefmann, V. Rödl e R. Yuster. The algorithmic aspects of the regularity lemma. J. Algorithms, 16(1):80-109, 1994. 2, 44

[AN06] N. Alon e A. Naor. Approximating the cut-norm via Grothendieck's inequality. SIAM J. Comput., 35(4):787-803 (electronic), 2006. 1, 2, 16, 33, 34, 37, 41, 45, 47

[AS08] N. Alon e J. H. Spencer. The probabilistic method. Wiley-Interscience Series in Discrete Mathematics and Optimization. John Wiley \& Sons, Inc., Hoboken, NJ, third edição, 2008. With an appendix on the life and work of Paul Erdôs. 26

[Ba196] V. Balakrishnan, editor. Linear matrix inequalities in control theory and applications. John Wiley \& Sons, Ltd., Chichester, 1996. Internat. J. Robust Nonlinear Control 6 (1996), no. 9-10. 9

[BM08] J. A. Bondy e U. S. R. Murty. Graph theory, volume 244 of Graduate Texts in Mathematics. Springer, New York, 2008. 14

[BMMN11] M. Braverman, K. Makarychev, Y. Makarychev e A. Naor. The Grothendieck constant is strictly smaller than Krivine's bound. Em 2011 IEEE 52nd Annual Symposium on Foundations of Computer Science-FOCS 2011, páginas 453-462. IEEE Computer Soc., Los Alamitos, CA, 2011. 34

[Bol79] B. Bollobás. Graph theory, volume 63 of Graduate Texts in Mathematics. SpringerVerlag, New York, 1979. An introductory course. 14

[Bol98] B. Bollobás. Modern graph theory, volume 184 of Graduate Texts in Mathematics. Springer-Verlag, New York, 1998. 14

[BTN02] A. Ben-Tal e A. Nemirovski. On tractable approximations of uncertain linear matrix inequalities affected by interval uncertainty. SIAM J. Optim., 12(3):811-833 (electronic), 2002. 2

[CPTW04] R. Cleve, Høyer. P., B. Toner e J. Watrous. Consequences and limits of nonlocal strategies. Em In Proceedings of the 19th IEEE Conference on Computational Complexity, páginas 236-249, 2004. 34

[Die10] R. Diestel. Graph theory, volume 173 of Graduate Texts in Mathematics. Springer, Heidelberg, fourth edição, 2010. 14, 18

$\left[\mathrm{DKM}^{+} 12\right]$ D. Dellamonica, S. Kalyanasundaram, D. Martin, V. Rödl e A. Shapira. A deterministic algorithm for the Frieze-Kannan regularity lemma. SIAM J. Discrete Math., 26(1):15-29, 2012. 2, 47 
[dKVMW00] E. de Klerk, H. Van Maaren e J. P. Warners. Relaxations of the satisfiability problem using semidefinite programming. J. Automat. Reason., 24(1-2):37-65, 2000. 9

[DLR95] R. A. Duke, H. Lefmann e V. Rödl. A fast approximation algorithm for computing the frequencies of subgraphs in a given graph. SIAM J. Comput., 24(3):598-620, 1995. 44

[ET36] P. Erdôs e P. Turán. On Some Sequences of Integers. J. London Math. Soc., S111(4):261, 1936. 1, 44

[FK96] A. Frieze e R. Kannan. The regularity lemma and approximation schemes for dense problems. Em 37th Annual Symposium on Foundations of Computer Science (Burlington, VT, 1996), páginas 12-20. IEEE Comput. Soc. Press, Los Alamitos, CA, 1996. 1, 2, 47

[FK99] A. Frieze e R. Kannan. Quick approximation to matrices and applications. Combinatorica, 19(2):175-220, 1999. 21, 46, 48

[GGU72] M. R. Garey, R. L. Graham e J. D. Ullman. Worst-case analysis of memory allocation algorithms. Em Proceedings of the Fourth Annual ACM Symposium on Theory of Computing, STOC '72, páginas 143-150, New York, NY, USA, 1972. ACM. 18

[GJ78] M. R. Garey e D. S. Johnson. "Strong" NP-completeness results: motivation, examples, and implications. J. Assoc. Comput. Mach., 25(3):499-508, 1978. 18

[GJS76] M. R. Garey, D. S. Johnson e L. Stockmeyer. Some simplified NP-complete graph problems. Theoret. Comput. Sci., 1(3):237-267, 1976. 19

[GLS81] M. Grötschel, L. Lovász e A. Schrijver. The ellipsoid method and its consequences in combinatorial optimization. Combinatorica, 1(2):169-197, 1981. 2, 11, 12, 13

[GLS93] M. Grötschel, L. Lovász e A. Schrijver. Geometric algorithms and combinatorial optimization, volume 2 of Algorithms and Combinatorics. Springer-Verlag, Berlin, second edição, 1993. 12

[GM12] B. Gärtner e J. Matoušek. Approximation algorithms and semidefinite programming. Springer, Heidelberg, 2012. 10

[Gon07] T. F. Gonzalez, editor. Handbook of approximation algorithms and metaheuristics. Chapman \& Hall/CRC Computer and Information Science Series. Chapman \& Hall/CRC, Boca Raton, FL, 2007. 18

[Gow97] W. T. Gowers. Lower bounds of tower type for Szemerédi's uniformity lemma. Geom. Funct. Anal., 7(2):322-337, 1997. 1

[Gra66] R. L. Graham. Bounds for certain multiprocessing anomalies. Bell System Technical Journal, 45:1563-1581, 1966. 17

[Gro53] A. Grothendieck. Résumé de la théorie métrique des produits tensoriels topologiques. Bol. Soc. Mat. São Paulo, 8:1-79, 1953. 1, 25, 33, 34

[GvL13] G. H. Golub e C. F. van Loan. Matrix computations. Johns Hopkins Studies in the Mathematical Sciences. Johns Hopkins University Press, Baltimore, MD, fourth edição, 2013. 6, 7

[GW95] M. X. Goemans e D. P. Williamson. Improved approximation algorithms for maximum cut and satisfiability problems using semidefinite programming. J. Assoc. Comput. Mach., 42(6):1115-1145, 1995. 9, 16, 19, 43 
[Had75] F. Hadlock. Finding a maximum cut of a planar graph in polynomial time. SIAM J. Comput., 4(3):221-225, 1975. 19

[Hås01] J. Håstad. Some optimal inapproximability results. J. ACM, 48(4):798-859, 2001. 19

[HJ13] R. A. Horn e C. R. Johnson. Matrix analysis. Cambridge University Press, Cambridge, second edição, 2013. 5

[HK71] K. Hoffman e R. Kunze. Linear algebra. Second edition. Prentice-Hall, Inc., Englewood Cliffs, N.J., 1971. 4, 5

[HL96] T. Hofmeister e H. Lefmann. A combinatorial design approach to MAXCUT. Em Proceedings of the Seventh International Conference on Random Structures and Algorithms (Atlanta, GA, 1995), volume 9, páginas 163-175, 1996. 19

[HV91] D. J. Haglin e S. M. Venkatesan. Approximation and intractability results for the maximum cut problem and its variants. IEEE Trans. Comput., 40(1):110-113, 1991. 19

[Kar72] R. M. Karp. Reducibility among combinatorial problems. Em Complexity of computer computations (Proc. Sympos., IBM Thomas J. Watson Res. Center, Yorktown Heights, N.Y., 1972), páginas 85-103. Plenum, New York, 1972. 15, 19

[Kho02] S. Khot. On the power of unique 2-prover 1-round games. Em Proceedings of the Thirty-Fourth Annual ACM Symposium on Theory of Computing, páginas 767-775. ACM, New York, 2002. 34

[KN08] S. Khot e A. Naor. Linear equations modulo 2 and the $L_{1}$ diameter of convex bodies. SIAM J. Comput., 38(4):1448-1463, 2008. 2

[KN09] S. Khot e A. Naor. Approximate kernel clustering. Mathematika, 55(1-2):129-165, 2009. 2

[KN12] S. Khot e A. Naor. Grothendieck-type inequalities in combinatorial optimization. Comm. Pure Appl. Math., 65(7):992-1035, 2012. 2, 33

[KN13] S. Khot e A. Naor. Sharp kernel clustering algorithms and their associated Grothendieck inequalities. Random Structures Algorithms, 42(3):269-300, 2013. 2

[KO09] S. Khot e R. O'Donnell. SDP gaps and UGC-hardness for max-cut-gain. Theory Comput., 5:83-117, 2009. 2

[Kön01] H. König. On an extremal problem originating in questions of unconditional convergence. Em Recent progress in multivariate approximation (Witten-Bommerholz, 2000), volume 137 of Internat. Ser. Numer. Math., páginas 185-192. Birkhäuser, Basel, 2001. 34

[Kri77] J.-L. Krivine. Sur la constante de Grothendieck. C. R. Acad. Sci. Paris Sér. A-B, 284(8):A445-A446, 1977. 34

[KRT03] Y. Kohayakawa, V. Rödl e L. Thoma. An optimal algorithm for checking regularity. SIAM J. Comput., 32(5):1210-1235, 2003. 2, 45

[KS95] Y. Kohayakawa e J.A. Soares. Demonstraçôes Transparentes e a Impossibilidade de Aproximaçôes. XX Colóquio Brasileiro de Matemática, IMPA, 1995. 14

[KS96] J. Komlós e M. Simonovits. Szemerédi's regularity lemma and its applications in graph theory. Em Combinatorics, Paul Erdôs is eighty, Vol. 2 (Keszthely, 1993), volume 2 of Bolyai Soc. Math. Stud., páginas 295-352. János Bolyai Math. Soc., Budapest, 1996. 44 
[Lan87] S. Lang. Linear algebra. Undergraduate Texts in Mathematics. Springer-Verlag, New York, third edição, 1987. 5

[Lov79] L. Lovász. On the Shannon capacity of a graph. IEEE Trans. Inform. Theory, 25(1):1-7, 1979. 9

[Lov03] L. Lovász. Semidefinite programs and combinatorial optimization. Em Recent advances in algorithms and combinatorics, volume 11 of CMS Books Math./Ouvrages Math. SMC, páginas 137-194. Springer, New York, 2003. 9, 10, 12

[LP68] J. Lindenstrauss e A. Pełczyński. Absolutely summing operators in $L_{p^{-}}$-spaces and their applications. Studia Math., 29:275-326, 1968. 33

[LP09] L. Lovász e M. D. Plummer. Matching theory. AMS Chelsea Publishing, Providence, RI, 2009. Corrected reprint of the 1986 original [MR0859549]. 2

[LS07] N. Linial e A. Shraibman. Lower bounds in communication complexity based on factorization norms. Em STOC'07-Proceedings of the 39th Annual ACM Symposium on Theory of Computing, páginas 699-708. ACM, New York, 2007. 2

[LV12] M. Laurent e F. Vallentin. Semidefinite optimization. Lecture Notes, available at http://page.mi.fu-berlin.de/fmario/sdp/laurentv.pdf, 2012. 10

[Meg01] A. Megretski. Relaxations of quadratic programs in operator theory and system analysis. Em Systems, approximation, singular integral operators, and related topics (Bordeaux, 2000), volume 129 of Oper. Theory Adv. Appl., páginas 365-392. Birkhäuser, Basel, 2001. 2

[Nes98] Yu. Nesterov. Semidefinite relaxation and nonconvex quadratic optimization. Optim. Methods Softw., 9(1-3):141-160, 1998. 2

[NRT99] A. Nemirovski, C. Roos e T. Terlaky. On maximization of quadratic form over intersection of ellipsoids with common center. Math. Program., 86(3, Ser. A):463-473, 1999. 2

[OD72] G. I. Orlova e Ya. G. Dorfman. Finding the maximum cut in a graph. Izv. Akad. Nauk SSSR Tehn. Kibernet., (3):155-159, 1972. 19

[Pap94] C. H. Papadimitriou. Computational complexity. Addison-Wesley Publishing Company, Reading, MA, 1994. 14, 16

[PGWP $\left.{ }^{+} 08\right]$ D. Pérez-García, M. M. Wolf, C. Palazuelos, I. Villanueva e M. Junge. Unbounded violation of tripartite Bell inequalities. Comm. Math. Phys., 279(2):455-486, 2008. 1, 33

[Pis78] G. Pisier. Grothendieck's theorem for noncommutative $C^{*}$-algebras, with an appendix on Grothendieck's constants. J. Funct. Anal., 29(3):397-415, 1978. 34

[Pis86] G. Pisier. Factorization of linear operators and geometry of Banach spaces, volume 60 of CBMS Regional Conference Series in Mathematics. Published for the Conference Board of the Mathematical Sciences, Washington, DC; by the American Mathematical Society, Providence, RI, 1986. 1, 33

[Pis12] G. Pisier. Grothendieck's theorem, past and present. Bull. Amer. Math. Soc. (N.S.), 49(2):237-323, 2012. 33

[PT82] S. Poljak e D. Turzík. A polynomial algorithm for constructing a large bipartite subgraph, with an application to a satisfiability problem. Canad. J. Math., 34(3):519524, 1982. 19 
[PY91] C. H. Papadimitriou e M. Yannakakis. Optimization, approximation, and complexity classes. J. Comput. System Sci., 43(3):425-440, 1991. 17

[Ree91] J.A. Reeds. A new lower bound on the real grothendieck constant. Unpublished manuscript, available at http://www.dtc.umn.edu/reedsj/bound2.dvi, 1991. 34

[Rie74] R. E. Rietz. A proof of the Grothendieck inequality. Israel J. Math., 19:271-276, 1974. 30,34

[RS09] P. Raghavendra e D. Steurer. Towards computing the Grothendieck constant. Em Proceedings of the Twentieth Annual ACM-SIAM Symposium on Discrete Algorithms, páginas 525-534, Philadelphia, PA, 2009. SIAM. 2, 34

[RT10] O. Regev e B. Toner. Simulating quantum correlations with finite communication. SIAM J. Comput., 39(4):1562-1580, 2009/10. 2

[Sah75] S. Sahni. Approximate algorithms for the 0/1 knapsack problem. J. Assoc. Comput. Mach., 22:115-124, 1975. 17

[Sch86] Alexander Schrijver. Theory of linear and integer programming. Wiley-Interscience Series in Discrete Mathematics. John Wiley \& Sons, Ltd., Chichester, 1986. A WileyInterscience Publication. 11

[Sch03] A. Schrijver. Combinatorial optimization. Polyhedra and efficiency. Vol. A, volume 24 of Algorithms and Combinatorics. Springer-Verlag, Berlin, 2003. Paths, flows, matchings, Chapters 1-38. 8, 22

[SG76] S. Sahni e T. Gonzalez. P-complete approximation problems. J. Assoc. Comput. Mach., 23(3):555-565, 1976. 19

[Sze78] E. Szemerédi. Regular partitions of graphs. Em Problèmes combinatoires et théorie des graphes (Colloq. Internat. CNRS, Univ. Orsay, Orsay, 1976), volume 260 of Colloq. Internat. CNRS, páginas 399-401. CNRS, Paris, 1978. 1

[Tsi85] B. S. Tsirelson. Quantum analogues of Bell's inequalities. The case of two spatially divided domains. Zap. Nauchn. Sem. Leningrad. Otdel. Mat. Inst. Steklov. (LOMI), 142:174-194, 200, 1985. Problems of the theory of probability distributions, IX. 1, 2, 33

[TSKS04] A. Tanay, R. Sharan, M. Kupiec e R. Shamir. Revealing modularity and organization in the yeast molecular network by integrated analysis of highly heterogeneous genomewide data. PNAS, 101(9):2981-2986, 2004. 2

[TSS02] A. Tanay, R. Sharan e R. Shamir. Discovering statistically significant biclusters in gene expression data. Bioinformatics, 18(90001):S136-144, 2002. 2

[Vaz01] V. V. Vazirani. Approximation algorithms. Springer-Verlag, Berlin, 2001. 18

[Vit81] P. M. B. Vitányi. How well can a graph be n-colored? Discrete Math., 34(1):69-80, 1981. 19 